\title{
ZARISKI GEOMETRIES AND QUANTUM MECHANICS
}

by

Milan Zanussi

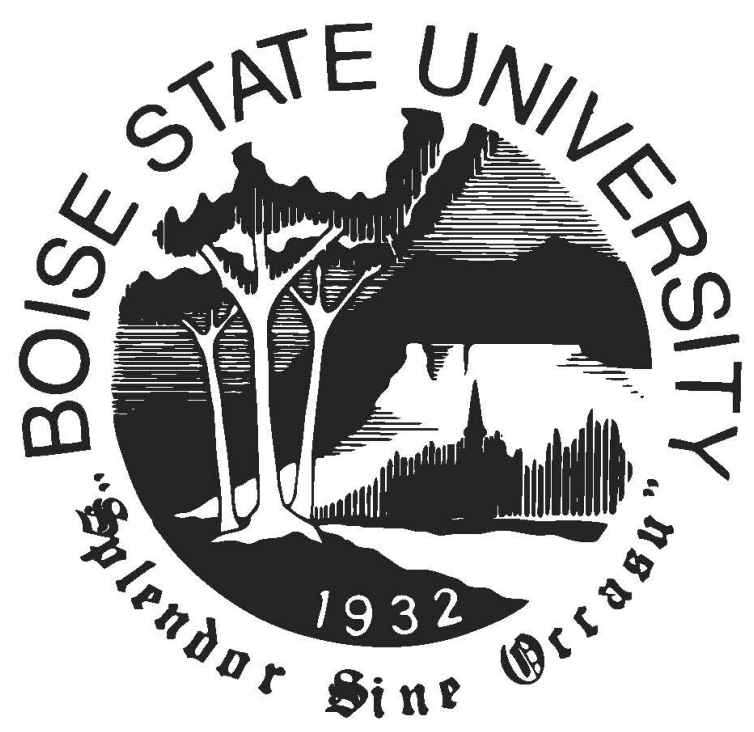

A thesis

submitted in partial fulfillment

of the requirements for the degree of

Master of Science in Mathematics

Boise State University

May 2021 
(C) 2021

Milan Zanussi

ALL RIGHTS RESERVED 
BOISE STATE UNIVERSITY GRADUATE COLLEGE

DEFENSE COMMITTEE AND FINAL READING APPROVALS

of the thesis submitted by

Milan Zanussi

Thesis Title: Zariski Geometries and Quantum Mechanics

Date of Final Oral Examination: $\quad 26$ March 2021

The following individuals read and discussed the thesis submitted by student Milan Zanussi, and they evaluated his presentation and response to questions during the final oral examination. They found that the student passed the final oral examination.

Samuel Coskey, Ph.D. Chair, Supervisory Committee

Uwe Kaiser, Ph.D. Member, Supervisory Committee

Zachariah Teitler, Ph.D. Member, Supervisory Committee

The final reading approval of the thesis was granted by Samuel Coskey, Ph.D., Chair of the Supervisory Committee. The thesis was approved by the Graduate College. 
Dedicated to my friends and family 


\section{ACKNOWLEDGMENTS}

I would like to express my gratitude to the Boise State University Department of Mathematics for giving me financial support to attend and complete my research at their institution through the teaching assistantship program. In this regard, I would specifically like to thank Jens Harlander for all the assistance he has given me in helping me succeed professionally and academically at Boise State. I would also like to express appreciation of my cohort of graduate students and my roommate, whose interactions have always felt very supportive and helped me feel more comfortable living in a new place. I'd also like to thank my friends and family, who have been extremely supportive of me throughout my Master's program, such as my parents, my brothers, and my friends in Tennessee and Virginia. Most importantly, I'd like to express my deepest gratitude towards my supervisor Sam Coskey, who has always been extremely supportive and encouraging of my endeavors into model theory and its deeper subjects; as well as my thesis committee members Uwe Kaiser and Zach Teitler, who have also offered plenty of support of the work I've done, as well as been willing to answer my volleys of questions about various related topics which appeared along the path of my research. 


\begin{abstract}
Model theory is the study of mathematical structures in terms of the logical relationships they define between their constituent objects. The logical relationships defined by these structures can be used to define topologies on the underlying sets. These topological structures will serve as a generalization of the notion of the Zariski topology from classical algebraic geometry. We will adapt properties and theorems from classical algebraic geometry to our topological structure setting. We will isolate a specific class of structures, called Zariski geometries, and demonstrate the main classification theorem of such structures. We will construct some Zariski structures where the classification fails by adding some noncommuting structure to a classical one. Finally we survey an application of these nonclassical Zariski structures to computation of formulas in quantum mechanics using a method of structural approximation developed by Boris Zilber.
\end{abstract}




\section{TABLE OF CONTENTS}

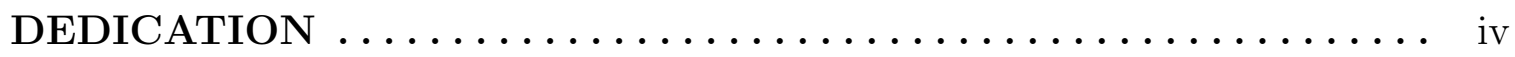

ACKNOWLEDGMENTS $\ldots \ldots \ldots \ldots \ldots \ldots \ldots \ldots \ldots \ldots \ldots \ldots$

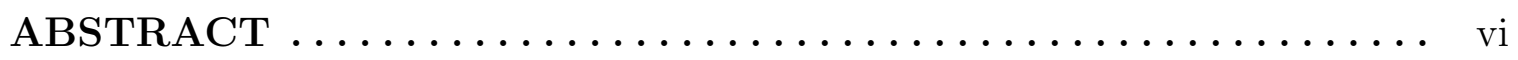

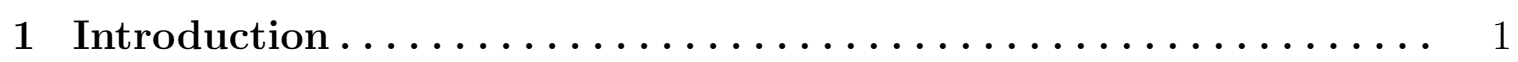

2 Model Theory Background $\ldots \ldots \ldots \ldots \ldots \ldots \ldots \ldots \ldots \ldots \ldots$

3 Topological and Zariski Structures $\ldots \ldots \ldots \ldots \ldots \ldots \ldots \ldots \ldots$

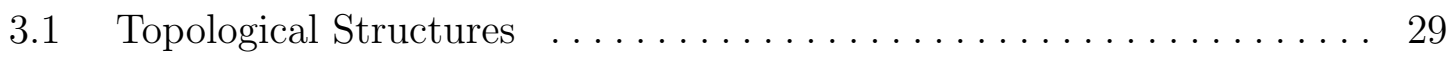

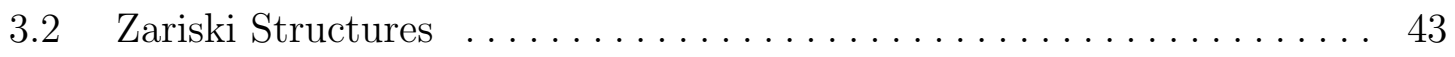

3.3 Elementary Extensions of Zariski Structures $\ldots \ldots \ldots \ldots \ldots \ldots .51$

3.4 Coverings of Zariski Structures $\ldots \ldots \ldots \ldots \ldots \ldots \ldots \ldots \ldots \ldots$

4 Classical Zariski Geometries $\ldots \ldots \ldots \ldots \ldots \ldots \ldots \ldots \ldots \ldots \ldots \ldots \ldots \ldots \ldots$

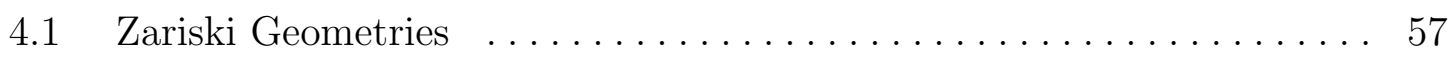

4.2 Coverings and Local Functions in Zariski Geometries ........... 62

4.3 Well-Behaved Kinds of Zariski Geometries . ................. 67

4.4 Curves, Branches, and Tangency $\ldots \ldots \ldots \ldots \ldots \ldots \ldots \ldots \ldots \ldots$

4.5 Obtaining the Z-field and Classification Theorem $\ldots \ldots \ldots \ldots \ldots .79$ 
5 Nonclassical Zariski Geometries.................... 91

5.1 An Example of Nonclassical Structures . . . . . . . . . . . . . . 91

5.2 Noncommutative Geometry for the Nonclassical Case . . . . . . . . . 95

6 Applications to Quantum Mechanics .................. 106

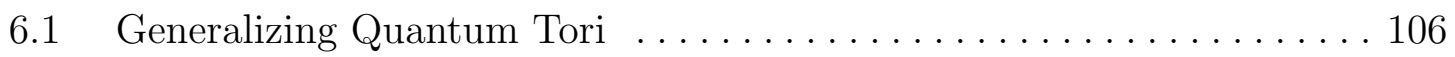

6.2 Structural Approximation $\ldots \ldots \ldots \ldots \ldots \ldots \ldots \ldots \ldots \ldots \ldots \ldots \ldots \ldots$

6.3 Dirac Calculus and Applying the Torus $\ldots \ldots \ldots \ldots \ldots \ldots \ldots \ldots$

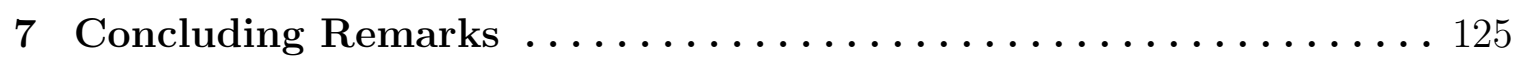

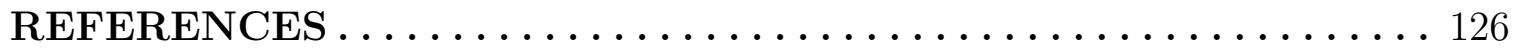




\section{CHAPTER 1}

\section{INTRODUCTION}

Physicists have not always used the most mathematically precise and rigorous techniques to compute formulas in physical models, but nevertheless physicists will sometimes manage to use these heuristic methods to reliably predict phenomena about the world. In particular, subdisciplines of physics such as quantum field theory have developed powerful and predictive results by using heuristic methods to compute non-convergent integrals and sums, as well as remove singularities from processes. An account of such methods can be found in [8].

On a philosophical basis, we might ask how we can simultaneously demand the sciences to provide rigorous arguments for their results, but allow for scientists to rely on such heuristic methods which essentially ignore the nature of the mathematical tools they use to support their arguments. On one hand, we could outright reject that any knowledge of the world has been obtained through these methods and arguments; but that the fact that the results accurately predicts reality might be an indicator that the statement is still true, despite the lack of affirmative knowledge that the claim is true. On the other hand, we might ask whether or not the mathematical structures we are using are the correct mathematical structures for modeling the situation, and whether or not some structure of our mathematical objects in our model come loaded with tacit assumptions that we are imposing on the world, but are unreflective of the 
actual world. If this is the case, then we can ask whether or not that structure is necessary for our model to describe the relationships between the objects in the world accurately.

One of the beliefs of the author that model theory - a branch of contemporary mathematical logic which studies mathematical structures in terms of the logical relationships they define between their constituent objects - could help provide a tool to take the latter approach to resolving the discrepencies between these scientific models and the heuristics used in their methods with the mathematics tools used to justify these methods. This appoach seems similar to the approach taken by Boris Zilber in [13], who claims that logicians have the advantage of not constraining themselves to any specific mathematical structure a priori when they do their mathematics. In this paper, Zilber uses model-theoretic tools which he developed in prior papers and pursuits - such as so-called Zariski structures and geometries - to try to compute some quantities from quantum mechanics that usually rely on such unrigorous heuristic methods. Zilber's prior work on Zariski geometries and their application to quantum mechanics will be the subject of our discussion.

These Zariski structures originate from prior work of Ehud Hrushovski and Boris Zilber in [4]. This work was motivated by a conjecture proposed by Zilber in model theory, which concerned the kinds of geometrical structures which could be endowed on so-called strongly minimal structures. Zilber conjectured that the canonical geometries that can be endowed on all strongly minimal structures are the geometries of either a algebraic type, linear type, or combinatorial type. Work from Hrushovski in [2] gave counter-examples to this trichotomy, and collaborative research between Hrushovski and Zilber developed Zariski geometries to find a class of structures where the trichotomy conjecture essentially holds. 
In Chapter 2, we will introduce some of the basic notation and tools from model theory that we need to motivate and develop our results. In particular, we will use examples from algebraic geometry to demonstrate the definitions given, which will hopefully elucidate how model theory can help us generalize methods from algebraic geometry. We will also introduce notation along the way which is commonly used in model theory, and will appear abundantly in our presentation. We will also briefly mention some of the motivating problems from model theory which inspired the particular model-theoretic structures developed in the later chapters.

In Chapter 3, we will develop some of our basic notions of topological structure theory, which will give us more tools to talk about models in a more geometric context. This notion of topological structure will be a generalization of the notion of defining a familiy of topologies such as the Zariski topologies on affine spaces from algebraic geometry. Furthermore, we will develop specific properties that will help us resolve some degeneracies in these structures, and help us in Chapter 4 to develop a notion of Zariski geometries, which are a specific kind of topological structure which possesses the imporantant model-theoretic properties that will interest us.

In Chapter 4, we will develop the theory of Zariski geometries, including theorems which allow us to define a notion of local functions in these topological structures. These local functions will help us define the notions of branches of curves and the tangency relations between them. Finally, we will build group and field structures on these equivalence classes of curves up to tangency, and demonstrate how these groups and fields essentially characterize the Zariski geometries in from which they are defined.

In Chapter 5, we will demonstrate that although some Zariski structures are nicely reducible to algebraic geometry over fields, we can construct certain kinds of 
nonclassical Zariski structures which are not reducible to fields. This construction will essentially rely on adjoining some kind of parameterized operator algebra to every point in a classical Zariski structure, and then demonstrating that when this operator algebra is noncommutative, the Zariski structure defined by the operator algebra will not be reducible to some kind of field-based structure.

Finally, in Chapter 6, we will survey an application of nonclassical Zariski structures by Boris Zilber in [13] to computing some formulas in quantum mechanics. In particular, Zilber's application is motivated by the observation that the operator algebras used to construct nonclassical Zariski structures can include a subclass of what he informally deems quantum algebra, since their commuting properties ressemble that of the Weyl commutation relation from physics. Furthermore, Zilber uses a notion of structural approximation to show that some models of particularly nice associated Zariski structures of quantum algebras can be used to approximate the actual associated Zariski structures used in physical models. We will introduce Zilber's method of structural approximation, which Zilber has described as a model-theoretical generalization of techniques such as the Gromov-Hausdorff limit or deformations of algebraic varieties to a general case of approximating mathematical objects. We will then use these tools to compute the kernel of a Feynman propagator of a free particle, which is a computation that usually relies on a heuristically computing a divergent integral. 


\section{CHAPTER 2}

\section{MODEL THEORY BACKGROUND}

Before we begin our exposition on Zariski structures, we should first investigate some of the basic tools of model theory that we will use throughout the later chapters. The model theory content of this chapter should give us a basic idea of what

model theory studies, as well as demonstrating to us how we can generalize some of the methodology of algebraic geometry to more broad classes of mathematical structures by discussing how these definitions and constructs and be truly recognized as generalizations of tools from algebraic geometry.

The study of model theory is primarily motivated by the study of the structure of mathematical objects. In particular, we think of the structure of a mathematical object as being described by the mathematical relationships it expresses between its contsituent objects. Hence, in model theory, we will devote most of our attention to the notion of languages, the symbols that we can use to express our language, the relationships our languages define in mathematical structures, and the properties of our mathematical structures which are preserved whenever we establish certain kinds of correspondences between different elements of different mathematical structures.

Definition 2.1 ( $\sigma$-structure (later called $\mathcal{L}$-structure)). Let $\sigma$ be a set of symbols intended to designate relations, functions, constants, and an equality symbols - where each symbol is associated with an arity which prescribes the number of arguments it 
accepts (for example, binary functions take two arguments, 5-ary relations take five arguments, constants are all 0 -ary etc.). Let $M$ be a set of elements and let $S \mapsto S_{M}$ be a function which maps symbols in $\sigma$ to objects over $M$ such that

(1.) The equality symbol in $=\epsilon \sigma$ is mapped to a maximally refined binary equivalence relation. That is $=_{M}$ consists of singleton equivalence classes of elements of $M$.

(2.) Every $n$-ary relation symbol $R \in \sigma$ (or function $f \in \sigma$ ) is mapped to an $n$-ary relation $R_{M} \subseteq M^{n}$ (or function is mapped to $f_{M}$ function from $M^{n}$ to $M$ )

(3.) Every constant $c \in \sigma$ is mapped to an element $c_{M} \in M$.

Then we call the triple $\left(M, \sigma,{ }_{M}\right)$ a $\sigma$-structure with domain $M$ and signature $\sigma$. For each symbol $S \in \sigma$, we say $S_{M}$ is the interpretation of $S$ in $M$.

Generally we will refer to a structure by its domain $M$, and assume that the signature is understood from context. We can also define the notion of a term, which is just a family of linguistic entitites in a $\sigma$-structure that is built starting from the base terms - variables symbols and constants of the structure - and then inductively applying functions to define new terms. We will give the following example so we can demonstrate how these tools from model theory will pertain to abstracting tools from algebraic geometry in later chapters.

Example 2.2 (Fields as structures). Let $\sigma=\left\{+, \cdot,-,^{-} \cdot 1,0,1\right\}$ and let these symbols be interpreted over the a field $F$ by the interpretations

(1.) + is interpreted the additive group operation on $F$, and - is interpreted as the multiplicative operation on $F$.

(2.) 0 is interpreted as the additive identity of $F$ and 1 is interpreted as the multiplicative identity of $F$. 
(3.) - is interpreted the additive inversion operation on $F$, and $\cdot^{-1}$ is interpreted as the multiplicative inversion operation for every element except 0 , which we will usually interpret by convention as $\left(0^{-1}\right)_{F}=0_{F}$ for simplicity.

(4.) Some examples of terms constructible in this structure are $1+1,1+x$, and $x \cdot(1+y \cdot y)^{-1}$. Typically, we abreviate $1+1$ as 2 , and also abbreviate

$$
x \cdot(1+y \cdot y)^{-1}=\frac{x}{1+y^{2}}
$$

by generally adopting the usual conventions for abbreviating terms from field theory. Note that if we choose a variable assignment of $y=0$, then the whole term becomes 0 instead of simply being undefined. The solution model theorists generally use for this issue is to disregard the semantics of this case when studying fields as structures, and acknowledge this term is not meaningful for this specific variable assignment.

Remark 2.3 (Multi-Sorted Structures). In (4) in the example of fields as a structure, another approach we could have taken to defining the multiplicative inverse of zero would be to develop a nortion of multi-sorted structures. The way a multi-sorted structure is defined is to modify our current definition of structure so that we can identify different sorts of objects, and then modify our notion of interpretation so that functions must specify the sorts over which they are defined. Sorts give us a way of subdividing the domains of our structures and indicating which arguments of our functions and relations should come from which sorts. In our fields example, we could have identified the sort $K^{*}=\{x \in K: x \neq 0\}$ and then indicated ${ }^{-1}: K^{*} \rightarrow K^{*}$ by saying $\cdot^{-1}$ has the sort type $\left(K^{*} ; K^{*}\right)$. This would have avoided the problem about the definition of $0^{-1}$ altogether, at the cost of working through the minutia. We will see 
some examples of many-sorted structures later, but we will not define them rigorously here since almost all of our structures will be single-sorted.

Before we start developing any logic on these structures, let us note the following definition

Definition 2.4 (Substructures and Extensions). Let $M$ be an $\mathcal{L}$ structure and let $D \subseteq M$ be a subset of $M$. We say that $D$, along with the interpretation of $M$ restricted to elements of $D$, is a substructure of $M$ if all of the constant symbols in $M$ belong to $D$ and if $D$ is closed under all of the interpretations of its function symbols. Conversely, we say that $M$ is an extension of $D$ in this scenario. This relationship is denoted $D \subseteq M$ in general.

Once we define the notion of a $\sigma$-structure, we note that we can start to perform some logical calculus on this structure. We can start evaluating the truth values of equality and relation statements, and then define general formulas by induction by taking conjunctions (and statements), disjunctions (or statements), negations, and quantifiers over variables applied to other formulas, and by interpreting the truth values of these operated formulas in the natural way prescribed by first-order logic. The collection of all these first-order formulas is the set $\mathcal{L}$, called the first-order language over the signature $\sigma$. Most model theory texts will use $\mathcal{L}$ to refer to $\sigma$, and thus will call structures $\mathcal{L}$-structures instead of $\sigma$-structures.

In terms of notation, we will usually write formulas using later letters in the Greek alphabet such as $\varphi, \psi, \rho$, etc. If a formula uses a variable $x$ outside the scope of any quantifiers that reference the variable name, we call the variable a free variable. Free variables force the truth value of a formula to be dependent on the choice of elements of $M$ substituted into them, so if $x_{1}, x_{2}, \ldots, x_{n}$ are free variables of a formula $\varphi$, 
then we will write the formula as $\varphi\left(x_{1}, \ldots, x_{n}\right)$ to highlight this dependency, and the substitution of a variable $x_{i}$ with an object $a_{i} \in M$ is denoted $\varphi\left(x_{1}, \ldots, a_{i}, \ldots, x_{n}\right)$. We will also usually abbreviate tuples as $\bar{x}=\left(x_{1}, x_{2}, \ldots, x_{n}\right)$ so that we can briefly write $\varphi\left(x_{1}, \ldots, x_{n}\right)$ as $\varphi(\bar{x})$.

A formula without free variables will be called a sentence. Sentences are precisely the formulas which we can directly assign truth values, and every formula can be turned into a sentence with appropriate substitutions of elements into each variable used in the formula. Let $\varphi$ be a sentence in $\mathcal{L}$, and let $M$ be a $\mathcal{L}$-structure. We denote that $\varphi$ is true in $M$, or that $M$ satisfies $\varphi$, by

$$
M \vDash \varphi
$$

If $\varphi(\bar{x})$ is an $\mathcal{L}$-formula instead, we say that $\varphi\left(x_{1}, x_{2}, \ldots, x_{n}\right)$ is satisfiable or consistent in $M$ if there is a tuple $\bar{a} \in M^{n}$ such that

$$
M \vDash \varphi(\bar{a})
$$

in which case, we say that $\bar{a}$ satisfies the formula $\varphi(\bar{x})$.

The notion of formulas and sentences are some of the most important objects of study in model theory because to some extent, the model theorist is most predominantly concerned with a mathematical structure as the mathematical relationships and descriptions of a domain of objects given by a language. This gives us the following flavor of equivalence relation in model theory, which can be described as saying two structures are logically indistinguishable.

Definition 2.5 (Elementary Equivalence, Substructures, and Extensions). Let $\mathcal{L}$ be 
a first-order language, and let $M_{1}$ and $M_{2}$ be two $\mathcal{L}$-structures. We say that $M_{1}$ and $M_{2}$ are elementarily equivalent if for every sentence $\varphi$ in $\mathcal{L}$ the following holds :

$$
M_{1} \vDash \varphi \Leftrightarrow M_{2} \vDash \varphi
$$

Furthermore, if $M_{1} \subseteq M_{2}$, then we say that $M_{1}$ is an elementary substructure of $M_{2}$ and that $M_{2}$ is an elementary extension of $M_{1}$. This relationship would be denoted $M_{1} \leqslant M_{2}$.

With our truth notion set in place, we can now briefly introduce the following definitions. The first definition will be a general formulation of the notion of homomorphism, which should be natural to understand for any mathematician with a prior background in any kind of abstract algebra. The second definition will be the model theorist's notion of axiomatization.

Definition 2.6 ( $\mathcal{L}$-homomorphism (or $\sigma$-homomorphism)). Let $\mathcal{L}$ be a language with signature $\sigma$. Let $M_{1}$ and $M_{2}$ be any two $\mathcal{L}$-structures, and let $F: M_{1} \rightarrow M_{2}$ be a mapping such that :

(1.) Let $c$ be a constant symbol in $\sigma$. Then $F\left(c_{M_{1}}\right)=c_{M_{2}}$

(2.) Let $f$ be an $n$-ary function symbol in $\sigma$. Then for each $a_{1}, \ldots, a_{n} \in M_{1}$ :

$$
F\left(f_{M_{1}}\left(a_{1}, a_{2}, \ldots, a_{n}\right)\right)=f_{M_{2}}\left(F\left(a_{1}\right), F\left(a_{2}\right), \ldots, F\left(a_{n}\right)\right)
$$

(3.) Let $R$ be an $n$-ary relation symbol in $\sigma$. Then for each $a_{1}, \ldots, a_{n} \in M_{1}$ :

$$
\left(a_{1}, a_{2}, \ldots, a_{n}\right) \in R_{M_{1}} \Longrightarrow\left(F\left(a_{1}\right), F\left(a_{2}\right), \ldots, F\left(a_{n}\right)\right) \in R_{M_{2}}
$$


Then we call $F$ an $\mathcal{L}$-homomorphism (or a $\sigma$-homomorphism) from $M_{1}$ to $M_{2}$. If $F$ is injective and the condition in (3) is an equivalence, rather than an implication, then $F$ is called an $\mathcal{L}$-embedding. If $F$ is a surjective $\mathcal{L}$-embedding, then it is called an $\mathcal{L}$-isomorphism.

The following proposition about $\mathcal{L}$-homomorphisms will be presented without proof, but we note that the theorem follows a fairly standard proof technique in model theory that involves performing induction on the complexity of formulas (ie, the number of conjunctions, disjunctions, negations, and quantifiers used). That being said, we tend to stratify formulas in all sorts of different ways depending on which places where the induction on formulas fails (such as failure to preserve universal statements or negations). For the proposition, the only formulas that need clarification are the following:

(1.) A quantifier-free formula is any formula which contains no instances of quantifiers.

(2.) For any formula $\varphi(\bar{x})$, we can find an equivalent formula (ie, true under precisely the same variable substitutions for a given model) $\psi(\bar{x})$ such that all occurrences of negation in $\psi(\bar{x})$ are applied directly to the atomic formulas (primitive relation statements and equality statements between terms). $\psi(\bar{x})$ is called the negation normal form of $\varphi(\bar{x}) \cdot \varphi(\bar{x})$ is called a positive formula if its negation normal form contains no instances of the negation symbol.

We now say that a map $F: M_{1} \rightarrow M_{2}$ preserves a formula $\varphi(\bar{x})$ if for every $\bar{a} \in M_{1}^{n}$ we have

$$
M_{1} \vDash \varphi(\bar{a}) \Longrightarrow M_{2} \vDash \varphi(F \bar{a})
$$

where $F \bar{a}=\left(F\left(a_{1}\right), F\left(a_{2}\right), \ldots, F\left(a_{n}\right)\right)$ 
Proposition 2.7. Let $\mathcal{L}$ be a first-order language, and let $F: M_{1} \rightarrow M_{2}$ be an $\mathcal{L}$ homomorphism. Then the following are true.

(1.) If $F$ is an $\mathcal{L}$-embedding, then $F$ preserves every $\varphi(\bar{x})$ formula in $\mathcal{L}$ of the form $\exists y_{1}, y_{2}, \ldots, y_{n}(\psi(\bar{x}, \bar{y}))$ where $\psi$ is quantifier-free.

(2.) If $F$ is a surjective $\mathcal{L}$-homomorphism, then $F$ preserves positive formulas in $\mathcal{L}$.

(3.) If $F$ is an $\mathcal{L}$-isomorphism, then $F$ preserves all formulas in $\mathcal{L}$

This proposition tells us a way to understand how to establish that some amounts of formulas are preserved when transferring from one model to another. In particular, we can establish that preserving the signature is stronger than preserving the firstorder logic statements between two structures.

Definition 2.8 ( $\mathcal{L}$-theory). Let $\mathcal{L}$ be a first-order language. A set of sentences $T$ from the language $\mathcal{L}$ is called an $\mathcal{L}$-theory. We say that an $\mathcal{L}$-structure $M$ is a model of $T$ if $M \vDash T$, that is $M \vDash \varphi$ for each $\varphi \in T$. The class of all models of $T$ will be denoted $\operatorname{Mod}(T)$. Furthermore, the following properties can be used to define a $\mathcal{L}$-theory

Example 2.9 (Theories of Fields and Algebraically Closed Fields). Let $\mathcal{L}$ be the language of fields described in the previous example. The theory of fields is given by the usual field axioms, stated in terms of of the language of $\mathcal{L}$. Furthermore, we can add the following sentence for each $n \in \mathbb{Z}^{+}$

$$
\forall a_{0}, a_{1}, \ldots, a_{n} \exists x\left(a_{n} x^{n}+\ldots+a_{1} x+a_{0}=0\right)
$$

to the theory of fields to create the theory of algebraically closed fields, denoted $A C F$. We may also specify a theory of fields of a particular characteristic $p$ by adjoining the 
sentence

$$
\sum_{i<p} 1=0
$$

Remark 2.10. When we've defined a notion of a theory for structures, we may be inclined in some instances to decide whether or not two different languages $\mathcal{L}_{1}$ and $\mathcal{L}_{2}$ define the same kinds of theories. There is a sense in which this notion of translating between structures can be studied, but there will not be any deeper investigation here. Instead, we will use one fact about such translations that will make some later definitions more elegant to phrase : every language $\mathcal{L}$ has an alternative language $\hat{\mathcal{L}}$ and theory $\hat{T}$ such that $\hat{\mathcal{L}}$ is a purely relational language and $\hat{T}$ is a theory that describes some of the relation symbols as defining functions and constants. To provide a quick example of what we mean, suppose that $c$ is a constant in $\mathcal{L}$, then we can replace $c$ with the unary relation $I s_{c}(x)$, and add the following sentence to $\hat{T}$ :

$$
\exists x\left(I s_{c}(x) \wedge \forall y\left(I s_{c}(y) \Longrightarrow x=y\right)\right)
$$

This will mean that if $M$ is an $\mathcal{L}$-structure, then we can define a $\hat{\mathcal{L}}$-model of the theory $\hat{T}$ on the domain $M$ that omits the constant symbol $c$, but now identifies its interpreted object $c_{M}$ as the unique object satisfying the predicate $I s_{c}(x)$ according to the theory $\hat{T}$. Of course, there's a similar admissibility condition for expressing whenever an $(n+1)$-ary relation defines an $n$-ary function, but we will not write it here. The moral of the story is that we can eliminate function and constant symbols in general model theory, just so long as we make sure to prescribe certain relations as function and constant symbols in our relational language.

We will call a theory complete if and only if it characterizes all of the facts that can be characterized about a structure with respect to the first-order language $\mathcal{L}$. In 
more precise terms, let

$$
\operatorname{Th}(M)=\{\varphi: \varphi \text { is an } \mathcal{L} \text {-sentence and } M \vDash \varphi\}
$$

be the (complete) first-order theory of a structure $M$. Then we say that $T$ is complete if and only if there is an $\mathcal{L}$-structure $M$ such that $N \vDash T$ if and only if $\operatorname{Th}(N)=$ $\operatorname{Th}(M)$. Note that this can also read that $T$ is complete if and only if $M \equiv N$ for each pair of models $M, N$ of $T$.

One final thing to notice about formulas is that we can have partial substitutions of elements of $M$ into formulas, but elements of $M$ are not necessarily symbols in the language used to define formulas over $M$. There are some technical details here that have been buried, but a standard introductory textbook on model theory such as [5] will include how these technical details are sorted out. The main idea is that these are technically formulas in language $\mathcal{L}$ adjoined with parameters in a set $A \subseteq M$, which is usually denoted $\mathcal{L}(A)$. Regardless, they can be shown to have natural methods of truth valuation in the original language $\mathcal{L}$ so long as we keep our discussions over a fixed $\mathcal{L}$-structure $M$. We will denote a formula which uses variables $x_{1}, \ldots, x_{n}$ and parameters $a_{1}, \ldots, a_{m} \in M$ by

$$
\varphi\left(x_{1}, \ldots, x_{n} ; a_{1}, \ldots, a_{n}\right)=\varphi(\bar{x} ; \bar{a})
$$

or some other variation of this notation, which we hope will be readily understood from context.

Definition 2.11 (Definable Sets and Relations). Given a subset $A \subseteq M^{n}$, we say that $A$ is a definable $n$-ary relation over parameters $\bar{b} \in M^{m}$ (definable set if $n=1$ ) of the 
$\mathcal{L}$-structure $M$ if there is an $\mathcal{L}(\bar{b})$-formula $\varphi\left(x_{1}, \ldots, x_{n} ; b_{1}, b_{2}, \ldots, b_{m}\right)$ such that

$$
A=\left\{\bar{a} \in M^{n}: M \vDash \varphi(\bar{a} ; \bar{b})\right\}
$$

In this case, we say that $A$ is defined by the formula $\varphi(\bar{x} ; \bar{a})$. Sometimes we will denote this definable set as

$$
A=\varphi\left(M^{n}, \bar{b}\right)=\varphi(M, \bar{b})
$$

where in the second notation, we are ignoring the number of variables used in the definition.

Example 2.12 (Affine Varieties as Definable Sets). Let $K$ be a field, and let $p(\bar{x}) \epsilon$ $K\left[x_{1}, x_{2}, \ldots, x_{n}\right]$ be a polynomial over $K$. The set

$$
D=\left\{\bar{x} \in K^{n}: p(\bar{x})=0\right\}
$$

is definable in $K$ fairly trivially, since $p(\bar{x})=0$ is a formula in the language of fields with parameters from $K$. Anyone with a background in algebraic geometry will recognize that these sets are called the affine varieties over $K$. As we will see later, these sets will be an excellent case study motivating example for the topology on $\mathbb{A}^{n}(K)=K^{n}$ ( which we will later call affine space ).

The study of definable sets is a pivotal theme of model theory - primarily because some of the structure of definable sets. In particular, we will discuss two notions which are used frequently in model theory, and these two notions in particular will relate to the specific tools of model theory we wish to investigate. 
Definition 2.13 (Minimal Sets and Structures). Let $M$ be an $\mathcal{L}$-structure, and let $D \subseteq M$ be a definable subset of $M$. If $D$ is an infinite subset such that for every other definable set $S$ either $D \cap S$ is finite or $D \backslash S$ is finite (or, in other words, $D \cap S$ is cofinite), then we say $D$ is a minimal subset in $M$. If $D=M$, then we say that $M$ is a minimal structure.

Essentially, a minimal structure is one where all of the definable subsets can be identified as essentially equivalent to some set with parameters which only uses Boolean combinations of atomics formulas which only use the equality symbols. We will come back to these kinds of sets later, but these sets will later be interesting for some geometrical properties we can associate with them.

We now note another piece of terminology that we will sometimes use when discussing families of definable sets (and equivalently, the formulas that describe them).

Definition 2.14 ((Finitely-)Consistent Family). Let $\mathcal{D}$ be a family of definable sets of an $\mathcal{L}$-structure $M$. We say that $\mathcal{D}$ is finitely consistent if for every $n \in \mathbb{Z}^{+}$and $D_{1}, D_{2}, \ldots D_{n} \in \mathcal{D}$, we have that $D_{1} \cap D_{2} \cap \ldots \cap D_{n}$ is not empty. This definition can also be expanded to families of definable relations as well.

A special case of finitely consistent families are finitely-consistent theories. What makes finitely consistent

Theorem 2.15 (Compactness Theorem). Let $T$ be a first-order $\mathcal{L}$-theory. $T$ is consistent if and only if $T$ is finitely consistent.

This theorem highlights a general technique that model theorists can use in firstorder logic. We can build new theories from old ones by taking a consistent theory $T$, 
such as the theory $\operatorname{Th}(M)=\{\varphi: M \vDash \varphi\}$, and then adjoin some new sentences to the theory. Then all that needs to be show is that every finite subset of new sentences adjoined to the theory are consistent with any finite subset of sentences of $T$. If this can be proven, then you can guarantee the existence of a model which satisfies the theory $T$ along with whichever sentences we adjoined to the theory.

Definition 2.16 (Types). Let $\bar{b} \in M^{n}$ and let $A \subseteq M$. We say that the complete $n$-type of $\bar{b}$ over $A$ in $M$ is the set of $\mathcal{L}$-formulas

$$
\operatorname{tp}_{M}(\bar{b} / A)=\left\{\varphi(\bar{x} ; \bar{a}): \bar{a} \in M^{k} \text { for some } k \in \mathbb{Z}^{+} \text {and } M \vDash \varphi(\bar{b} ; \bar{a})\right\}
$$

Furthermore, consider a set of $\mathcal{L}$-formulas $p\left(x_{1}, x_{2}, \ldots, x_{n}\right)$, each with parameters from $A$ in $M . p(\bar{x})$ is called an $n$-type over $A$ in $M$ if there is an elementary extension ${ }^{*} M \geqslant M$ and an element $\bar{b} \in{ }^{*} M^{n}$ such that $p(\bar{x}) \subseteq \operatorname{tp}^{*} M(\bar{b} / A)$.

Remark 2.17 (Types and Compactness). We note that the definition of a type basically says that a type is any set of formulas about some tuple in a structure which can be extended to a complete description of that object in an extended structure. In some ways, this means that a type is a slight generalization of the notion of an element to some capacity, and it carries with it a more fundamentally model-theoretical character - a type is the description of an object whose existence is consistent with the rest of the structure in which the type is specified. Some classical examples of types are objects like real numbers in the algebraic numbers, which can be specified by types which indicate sequences of nested algebraic (rational in particular) intervals that converge to them, but may not be realized as any particular algebraic numbers (such as $\pi$ for example). 
With this in mind, we will sometimes use the following corollary of the compactness theorem : a set of formulas over $n$ variables is an $n$-type if and only if the set of formulas is finitely consistent. By compactness, this means we can adjoin a tuple of $n$-many constants to realize the type, and the finite consistency will allow us to make sure that the extension exists to realize that type.

Given the notion of a type, we may also think that, mathematically, it would be nice for us to only work in structures where we can make sure that all of these theoretical objects posited by types are actually realized in a structure. That way our structure can perform its deductions with tangible objects which realize these types, as opposed to simply letting these describable pieces of structure go unrepresented as specific objects in the structure. The following notion captures the kinds of structures we would like to study, but they are rarely encountered naturally outside of mathematical logic and model theory.

Definition 2.18 (Saturation). Let $M$ be an $\mathcal{L}$-structure. We will call $M \kappa$-saturated for a cardinal $\kappa$ if for every subset $A \subseteq M$ such that $|A|<\kappa$, all of the $n$-types over $A$ are realized for each $n \in \mathbb{Z}^{+}$.

As one might expect, there are canonical ways of transfinitely inductively adding all of the types over sets with cardinality less or equal to $\kappa$ using theorems like compactness, given some extra conditions, though. For the statement of the next theorem, note that $|\mathcal{L}|$ represents the number of formulas in the first-order language $\mathcal{L}$ up to change of variables. This set is always infinte, and its minimum possible value is $\omega$ in general (in the case where the signature is finite or countable).

Proposition 2.19 (Existence of Saturated Extensions). Let $M$ be an $\mathcal{L}$-structure where $\mathcal{L}$ is a first-order language. Furthermore, let $\lambda \geq|\mathcal{L}|$. Then $M$ has an elementary 
extension to a $\lambda^{+}$-saturated $\mathcal{L}$-structure ${ }^{*} M$ of cardinality $\left.\left.\right|^{*} M|\leq| M\right|^{\lambda}$.

Reference. A proof of this theorem can be found in [1] as Corollary 8.2.2.

In the context of model theory, we generally assume that whatever structure we work with is sufficiently saturated for our purposes. These structures go by many different names, such as big models, monster models, and universal domains. We will call them universal domains from here forward. Working in such an extension gives us the desired goal of having explicit representatives for each type with which we are concerned, as guaranteed by a complete first-order theory.

We will now turn out attention to a model-theoretic tool which will be used in chapter 6 when we develop our notion of structural approximation. This tool will be called an ultraproduct, and it has a wide variety of uses in model theory. One of its most important uses, on top of our application, is that ultraproducts can be used to build construct saturated structures if their parameters are chosen just right, but we will not go into detail about this here. Before we discuss the ultraproduct, however, we should first define a direct product.

Definition 2.20 (Direct Product). Let $\left\{M_{i}: i \in I\right\}$ be an indexed family of $\mathcal{L}$ structures. The direct product of this family of structures is the structure $N=\prod_{i \in I} M_{i}$ defined by letting the domain of the structure be the set of choice functions $a: I \rightarrow$ $\cup_{i \in I} M_{i}$ (ie, for all $i \in I$, we have $\bar{x}(i) \in M_{i}$ ), and define the interpretation such that

(1.) If $a, b \in N$, we say $a=\bar{b}$ if and only if $a(i)=b(i)$ for all $i \in I$.

(2.) If $a \in N$ and $c$ is a constant symbol, then $a=c_{N}$ if and only if $a(i)=c_{M_{i}}$ for each $i \in I$. 
(3.) Let $f$ be an $n$-ary function symbol. Then for all $a_{1}, a_{2}, \ldots, a_{n}$ and $b$ in $N$, we let

$f_{N}\left(a_{1}, a_{2}, \ldots, a_{n}\right)=b$ if and only if $f_{M_{i}}\left(a_{1}(i), a_{2}(i), \ldots, a_{n}(i)\right)=b(i)$ for each $i \in I$

(4.) Let $R$ be an $n$-ary relation symbol. Then for $a_{1}, a_{2}, \ldots, a_{n} \in N$ let

$$
\left(a_{1}, a_{2}, \ldots, a_{n}\right) \in R_{N} \text { if and only if }\left(a_{1}(i), a_{2}(i), \ldots, a_{n}(i)\right) \in R_{M_{i}} \text { for each } i \in I
$$

With the notion of a product defined, we can start defining ways of taking some kinds of quotients of this product structure. In particular, recall the notion of a filter from order theory. Let us take a filter $\mathcal{F}$ on the Boolean algebra of subsets of $I$. In particular, let us define the equivalence relation on $\prod_{i \in I} M_{i}$ by saying for $a, b \in \prod_{i \in I} M_{i}$ that

$$
a \sim b \quad \text { if and only if } \quad\left\{i \in I: M_{i} \vDash a(i)=b(i)\right\} \in \mathcal{F}
$$

We can now take the set of equivalence classes under this equivalence relations and use it as the domain of a new structure. In particular, let us assume that $\mathcal{F}=\mathcal{U}$ is an ultrafilter of subsets of $I$ - which is simply a maximal filter. Since the set of subsets of $I$ form a Boolean algebra, it is actually an equivalent condition for $\mathcal{U}$ to have the property that

$$
X \notin \mathcal{U} \quad \text { if and only if } \quad X^{c}=I \backslash X \in \mathcal{U}
$$

Now for the following definition, let us go ahead and introduce the notation that for every $\varphi$ statement in $\mathcal{L}$, the Boolean value of $\varphi$ in $\prod_{i \in I} M_{i}$ is

$$
\|\varphi\|=\left\{i \in I: M_{i} \vDash \varphi\right\}
$$


Let us also denote our equivalence class for an element $a$ under the equivalence relation (2.1) by $a / \mathcal{F}$ where $\mathcal{F}$ is the filter used to define the equivalence relation.

Definition 2.21 (Ultraproduct). Let $\left\{M_{i}: i \in I\right\}$ be an indexed family of $\mathcal{L}$-structures, and let $\mathcal{U}$ be an ultrafilter of subsets of $I$. Then the ultraproduct of $\left\{M_{i}\right\}_{i \in I}$ along $\mathcal{U}$ is an $\mathcal{L}$-structure with a domain equal to $N=\prod_{i \in I} M_{i} / \mathcal{U}$, the set of all equivalence classes defined by the equivalence relation in (2.1). We also define the interpretation of $N$ by the following

(1.) Equality is defined by two equivalence classes being the same one.

(2.) Let $c$ be a constant symbol. Then we say that

$$
c_{N}=a / \mathcal{U} \text { where } \hat{a} \in a / \mathcal{U} \text { we have } a(i)=c_{M_{i}} \text { for all } i \in I
$$

(3.) Let $f$ be an $n$-ary function symbol and let $a_{1}, a_{2}, \ldots, a_{n} \in \prod_{i \in I} M_{i}$. Then we say that

$$
f_{N}\left(a_{1} / \mathcal{U}, \ldots, a_{n} / \mathcal{U}\right)=b / \mathcal{U} \text { where } f_{M_{i}}\left(a_{1}(i), \ldots, a_{n}(i)\right)=b(i) \text { for each } i \in I
$$

(4.) Let $R$ be an $n$-ary relation symbol and $a_{1}, a_{2}, \ldots, a_{n} \in \prod_{i \in I} M_{i}$. Then we say that

$$
\left(a_{1} / \mathcal{U}, \ldots, a_{n} / \mathcal{U}\right) \in R_{N} \text { if and only if }\left\|R\left(a_{1}, a_{2}, \ldots, a_{n}\right)\right\| \in \mathcal{U}
$$

With all of this machinery in place, we will go ahead and state one classical, and fairly simple, result about ultraproducts. 
Theorem 2.22 (Łoś's Theorem). Let $\left\{M_{i}\right\}_{i \in I}$ be a family of $\mathcal{L}$ structures, and let $N=$ $\prod_{I} M_{i} / \mathcal{U}$ be the ultraproduct of $\left\{M_{i}\right\}_{i \in I}$ along $\mathcal{U}$. Then for every formula $\varphi\left(x_{1}, \ldots, x_{n}\right)$ and any $a_{1}, \ldots, a_{n} \in \prod_{I} M_{i}$, the following holds

$$
N \vDash \varphi\left(a_{1} / \mathcal{U}, \ldots, a_{n} / \mathcal{U}\right) \quad \text { if and only if } \quad\left\|\varphi\left(a_{1}, \ldots, a_{n}\right)\right\| \in \mathcal{U}
$$

Outline. This theorem follows from a fairly simple proof by induction on the complexity of formulas. In particular, everything except for induction on negation symbols can be proven without the assumption that $\mathcal{U}$ is an ultrafilter, but then the fact that $\mathcal{U}$ is an ultrafilter proves that step of the induction trivially by $(2.2)$.

We will now turn our attention to our last tool, and with a biref discussion of some of the implications of this tool in model theory. This tool was originally developed to prove a famous theorem in model theory known as Morley's Categoricity Theorem. The theorem states that any theory with a countable language which is categorical in one uncountable cardinal must be categorical in all uncountable cardinals. The tool is meant to give a rough notion of dimension in the purely model-theoretical context.

Definition 2.23 (Morley Rank). Let $M$ be a sufficiently saturated $\mathcal{L}$-structure, and let $\varphi\left(x_{1}, \ldots, x_{n}\right)$ be a formula in $\mathcal{L}$. Let us define a function $r k$, whose output is either $-1, \infty$, or an ordinal, with the following relations

(1.) $\operatorname{rk}(\varphi) \geq 0$ if and only if $\varphi\left(M^{n}\right)$ is not empty.

(2.) For $\alpha+1$ a successor ordinal, we say that $\operatorname{rk}(\varphi) \geq \alpha+1$ if and only if there is a sequence $\left\{\psi_{k}\left(x_{1}, \ldots, x_{n}\right)\right\}_{k \in \omega}$ of formulas such that the family $\left\{\phi\left(M^{n}\right) \cap\right.$ $\left.\psi_{k}\left(M^{n}\right)\right\}_{k \in \omega}$ is pairwise disjoint, and consists of definable sets with $\operatorname{rk}\left(\phi \wedge \psi_{k}\right) \geq \alpha$ for each $k \in \omega$. 
(3.) For a limit ordinal $\lambda, \operatorname{rk}(\varphi) \geq \lambda$ if and only if $\operatorname{rk}(\varphi) \geq \alpha$ for each $\alpha<\lambda$.

We then say that $\operatorname{rk}(\varphi)=-1$ if $\varphi\left(M^{n}\right)$ is empty. Otherwise we say that, if it exists, we define

$$
\operatorname{rk}(\varphi)=\min \{\alpha: \alpha \text { is an ordinal, and } \operatorname{rk}(\varphi) \geq \alpha\}
$$

and if this value doesn't exist, then we define $\operatorname{rk}(\varphi)=\infty$. The output of the function $\operatorname{rk}(\varphi)$ is called the Morley rank of $\varphi$.

Of course, one thing we can notice from this definition is that we could have equivalently constructed the definition using definable sets instead of formulas, and we would have obtained essentially the same definition. In fact, let $D$ be a subset of $M$ defined by the formula $\varphi(\bar{x})$. Then we can define $\operatorname{rk}(D)=\operatorname{rk}(\varphi)$. We can also note that the definable set of a structure is $M=\{x \in M: x=x\}$, and so the Morley rank of a structure $\operatorname{rk}(M)$ is computed by the Morley rank of the formula $x=x$.

A common result in model theory to note is that $\omega$-saturation (or $\aleph_{0}$-saturation in cardinal notation) is sufficient to compute Morley rank. More precisely, Morley rank computed in an $\omega$-saturated extension of a structure is equal to the Morley rank computed in any $\kappa$-saturated extension of a structure for $\kappa \geq \omega$. This result can be found in $[5]$.

To solidify the idea that the Morley rank defines a dimension notion, we will mention the following list of properties found in [1] about Morley rank.

Proposition 2.24 (Properties of Morley Rank). Let $M$ be an $\omega$-saturated $\mathcal{L}$-structure, let $\varphi(\bar{x})$ and $\psi(\bar{x})$ be $\mathcal{L}$-formulas, and let $\chi\left(\bar{x}, y_{1}, y_{2}, \ldots, y_{n}\right)$ be an $\mathcal{L}$-formula without parameters.

(1.) If $M \vDash \forall \bar{x}(\varphi(\bar{x}) \rightarrow \psi(\bar{x}))$, then $\operatorname{rk}(\varphi) \leq \operatorname{rk}(\psi)$. 
(2.) $\operatorname{rk}(\varphi \vee \psi)=\max (\operatorname{rk}(\varphi), \operatorname{rk}(\psi))$

(3.) Let $c_{1}, c_{2}, \ldots, c_{n}$ be new constant symbols adjoined to $\mathcal{L}$ to make a new language. Let us consider two different interpretations $(M, \bar{a})$ and $(M, \bar{b})$ of this new language $\mathcal{L} \cup\left\{c_{1}, \ldots, c_{n}\right\}$ over domain $M$ such that $(M, \bar{a}) \vDash c_{i}=a_{i}$ and $(M, \bar{b}) \vDash c_{i}=b_{i}$ for all $i \leq n$. If $(M, \bar{a}) \equiv(M, \bar{b})$, then $\operatorname{rk}(\chi(\bar{x}, \bar{a}))=\operatorname{rk}(\chi(\bar{x}, \bar{b}))$

In this proposition, (1) says that taking definable subsets can only ever reduce Morley rank. In property (2), we see that the Morley rank of a union of two definable sets is equal to the maximum of their ranks. Lastly, (3) tells us that if we have any formula with parameters, then we can exchange those parameters for any other equivalent set of parameters to obtain a formula of the same rank. All of these have the flavor of the kinds of dimension notions that would be studied in algebraic geometry, such as the dimension of a variety. Indeed, in [5] it is shown that the dimension of an irreducible variety over an algebraically closed field is equal to its Morley rank.

Another notion associated with Morley rank is the idea that every definable set of Morley rank $\alpha$ has a maximal finite partition into other definable sets of Morley rank $\alpha$ - this follows almost immediately from the definition of Morley rank. We call the maximum size of these partitions the Morley degree of a formula, often denoted $\operatorname{deg}(\varphi)$ for a formula $\varphi(\bar{x})$. In algebraic geometry, this is analogous to the number of irreducible components of a variety.

Morley rank was initially developed as a tool in model theory to prove the following theorem, which can be found proven in [1] and [5]. The proof of this theorem relies on techniques which utilize some of the quasi-geometrical nature of Morley rank.

Theorem 2.25 (Morley's Categoricity Theorem). Let $T$ be a complete theory over $\mathcal{L}$. If $T$ is $\kappa$-categorical for some uncountable cardinal $\kappa$ (that is, a model $M \vDash T$ with 
$|M|=\kappa$ is unique up to $\mathcal{L}$-isomorphism), then $T$ is $\lambda$-categorical for all uncountable cardinals $\lambda$ (we say that $T$ is uncountably categorical).

Finally, with Morley rank defined, we will finish our model theory background discussion with a brief return to minimal sets, and note in particular that every definable set with Morley rank 1 and Morley degree 1 is a minimal set, since we know that if we take any definable subset, then that definable subset must either be Morley rank 0 (finite), or have a relative complement in the definable subset which is Morley rank 0 . However, we also note that not every minimal set will have Morley rank 1 and Morley degree 0 , since not every minimal set is defined in an $\omega$-saturated structure. This gives us into our following definition, which is a Morley rank definition for a stronger notion of minimality.

Definition 2.26 (Strong Minimality). We say that a formula $\varphi$ over $M$ (equivalently the set it defines) is strongly minimal if and only if its Morley rank and Morley degree are both 1 . In other words, $\varphi$ is strongly minimal if and only if it is minimal some $\omega$-saturated elementary extension of $M$.

Strong minimality is one of the first major classification properties of model theory which has been widely studied and mostly resolved. Some of the most important results about strong minimality pertain to how they can be endowed with a closure operation pregeometry structure, which is essentially a infinite matroid structure modified by an axiom ressembling the finite-character property of closure operations. The specific closure operation used is the following

Definition 2.27 (Algebraic Closure Operation). Let $M$ be an $\mathcal{L}$-structure, and let $D \subseteq M$ be a definable subset. We say that an object $a \in M$ is algebraic over $D$ is there is a formula $\varphi\left(x ; y_{1}, \ldots, y_{n}\right)$ and some parameters $b_{1}, b_{2}, \ldots, b_{n} \in D$ such that 
$M \vDash \varphi\left(a ; b_{1}, b_{2}, \ldots, b_{n}\right)$ and also $M$ models the assertion that there is only finitely many choices for $x$ that satisfy this formula with these parameters. We then define the algebraic closure of $D$ as the set

$$
\operatorname{acl}_{M}(D)=\{a \in M: a \text { is algebraic over } D\}
$$

In Chapter 9 of [1], there is a proof that the algebraic closure operation defines a pregeometry on any minimal subset of an $\mathcal{L}$-structure. The only property which is difficult to prove is the exchange property. Furthermore, the pregeometry allows us to define a notion of bases, which allows us to more easily classify strongly minimal structures, since we can develop a generalization of the theorem of matroids that allows us to determine if two matroids are isomorphic by finding an isomorphism between their bases.

While these structures have incredible properties, for a long time the only structures we knew to be strongly minimal were structures based on the three classical notions of dependence relations found in mathematics : algebraic dependence in fields, linear dependence over vector spaces, and combinatorial dependence of pairs of vertices in a graph. To clarify, by combinatorial dependence we mean the dependence relation defined by considering two vertices in a graph dependent if and only if they lie in the same connected component.

These types of dependencies, as well as their abundance in the class of strongly minimal structures, motivated the following conjecture proposed by Zilber as an initial proposal that such structures could be seen as being among the most logically perfect structures in first-order model theory, due to their nice classification properties. This conjecture was also proven in a weaker form by Zilber in [11]. The conjecture is 
summzarized as follows

Conjecture 2.28 (Zilber's Trichotomy Conjecture). The geometry of a minimal structure is isomorphic to one of the three classical types described in the preceding paragraphs.

This conjecture was disproven by Hrushovski in [2] which demonstrated that upon introducing some appropriate pre-dimension notion, one could define structures which could be used to define new kinds of strongly minimal sets whose geometries were not recognizable as any of the types listed.

With this in mind, Hrushovski and Zilber collaborated on identifying ways in which the Trichotomy conjecture could be salvaged. This led to the body of tools which will be presented in the next chapters, which were initially developed in the paper [4] to strengthen the assumptions of the Trichotomy conjecture and find a class of structures where the Trichotomy principle at least essentially or mostly holds. The core idea was to adapt the notion of a Zariski topology from algebraic geometry to studying more general topologies induced by definable sets of structures, and then pick a particularly nice set of topological properties which can control for any irregularities one might encounter when trying to classify strongly minimal structures. 


\section{CHAPTER 3}

\section{TOPOLOGICAL AND ZARISKI STRUCTURES}

In this chapter, we will develop the first main tools for studying the geometrical classification we would like to pursue in general model-theoretical structures. In particular, we will develop the notion of a topological structure - which is simply a structure where some subsystem of its definable sets are taken as the closed subsets of a topology, and generally should have its topology generated by the logic on these definable sets. We will then introduce some additional properties and constructs which will be useful for developing other interesting topological notions on on these structures, such as working towards developing a theory of infinitesimals in topological structures. Finally, we will isolate some of our most important properties to develop the notion of a (Noetherian) Zariski structure. Here the use of Noetherian is primarily used as a distinguishing name from another type of Zariski structure which will not be discussed, called an analytic Zariski structure - these are discussed in Chapter 6 of [14], however. The usual Zariski structures we consider will have a number of nice model-theoretical properties which will prepare much of our classification theory, but will not come to complete fruition until chapter 4 when we develop the notion of a Zariski geometry. 


\subsection{Topological Structures}

Before we directly pursue the general formulations of Zariski structures that we would like to identify the main way we will endow mathematical structures with topologies. In particular, our first definition will be seen as a vast generalization of the method used to define the Zariski topologies on affine spaces of a variety. Here, we will allow closed sets to be definable by any class of closed sets which happens to form a topological space.

Definition 3.1 (Topological Structure). Let $\mathcal{L}$ be a (relational) language and let $M$ be an $\mathcal{L}$-structure. Furthermore, let $\mathcal{C}$ be a family of definable subsets of $M$. We call $(M, \mathcal{C})$ a topological $\mathcal{L}$-structure with closed sets $\mathcal{C}$ if it satisfies the following axioms, which we will collectively refer to by the abbreviation $(L)$ :

(L1.) $\mathcal{C}$ is closed under intersections.

(L2.) $\mathcal{C}$ is closed under finite unions.

(L3.) The domain $M$ and the empty set $\varnothing$ are closed.

(L4.) The graph of equality $\Gamma_{e q}=\left\{(x, y) \in M^{2}: x=y\right\}$ is closed.

(L5.) Every singleton set of $M$ is closed.

(L6.) Cartesian products of closed sets are closed.

(L7.) Let $\sigma$ be a permuation of $\{1,2, \ldots, n\}$ and let $\pi_{\sigma}: M^{n} \rightarrow M^{n}$ be defined by

$$
\pi_{\sigma}\left(x_{1}, x_{2}, \ldots, x_{n}\right)=\left(x_{\sigma(1)}, x_{\sigma(2)}, \ldots, x_{\sigma(n)}\right)
$$

Then if $S \in \mathcal{C}$, then also $\pi_{\sigma}(S) \in \mathcal{C}$ (ie. $\mathcal{C}$ is closed under permutations of coordinates). 
(L8.) For any $\bar{a} \in M^{m}$ and any $S \subseteq M^{m+n}$ such that $S \in \mathcal{C}$ and is defined by the predicate $S\left(x_{1}, \ldots, x_{m}, y_{1}, \ldots, y_{n}\right)$, the fiber $S\left(\bar{a}, M^{n}\right)=\left\{\bar{b} \in M^{n}: M \vDash S(\bar{a}, \bar{b})\right\}$ over $\bar{a}$ is in $\mathcal{C}$.

To give a brief motivation for the axioms as they are, let us note the following. First, the axioms (L1), (L2), and (L3) are essentially the axioms of a topological space for each $\mathcal{C}_{n}$ family of closed $n$-ary formulas over $M$, meaning that topological structures define a family of topological spaces with one topology for each $M^{n}$ where $n \in \mathbb{Z}^{+}$. (L4) and (L5) tell us that the equality symbols and our choice of elements in the structure should always have some bearing on how the structure is defined (particularly, we include all sets defined by equality and parameter substitutions into equality). (L6) gives us as way of representing the process of conjoining two different formulas without necessarily mixing the use of variables between them. To give an example from this from algebraic geometry. Consider the two varieties

$$
V_{1}=\left\{\left(x_{1}, x_{2}\right) \in \mathbb{R}^{2}: x_{1}^{2}=x_{2}\right\} \text { and } V_{2}=\left\{\left(x_{1}, x_{2}, x_{3}\right) \in \mathbb{R}^{3}: y_{1}^{2}+y_{2}^{2}=y_{3}^{2}\right\}
$$

then we can take the conjunction of these two varieties without necessarily mixing their variables by taking the Cartesian product

$$
\begin{aligned}
V_{1} \times V_{2} & =\left\{\left(x_{1}, x_{2}, y_{1}, y_{2}, y_{3}\right) \in \mathbb{R}^{5}:\left(x_{1}, x_{2}\right) \in V_{1} \wedge\left(y_{1}, y_{2}, y_{3}\right) \in V_{2}\right\} \\
& =\left\{\left(x_{1}, x_{2}, y_{1}, y_{2}, y_{3}\right) \in \mathbb{R}^{5}: x_{1}^{2}=x_{2} \wedge y_{1}^{2}+y_{2}^{2}=y_{3}^{2}\right\}
\end{aligned}
$$

(L7) guarantees that our choice of which element of our tuples represents which variable in a formula has no effect on whether or not a set is considered closed. For example, without the axiom we could conceivably allow 


$$
\left\{(x, y) \in \mathbb{R}^{2}: x^{2}=y\right\}
$$

to be a closed set of $\mathbb{R}$, but also disallow

$$
\left\{(x, y) \in \mathbb{R}^{2}: y^{2}=x\right\}
$$

from being considered a definable subset, which seems inconsistent with the goal to induce the topology of our space from the logical properties of the closed sets of our structure. (L8) allows us to substitute variables with parameters into the formulas defining our closed subsets to obtain new closed sets. As a quick example, if we take the double-cone in $\mathbb{R}^{3}$, defined by our variety $V_{2}$ above, to be closed; then we must accept that every circle in $\mathbb{R}^{2}$ obtained using any real number $r$ by

$$
S_{r}^{1}=\left\{\left(x_{1}, x_{2}\right) \in \mathbb{R}^{2}:\left(x_{1}, x_{2}, r\right) \in V_{2}\right\}=\left\{\left(x_{1}, x_{2}\right) \in \mathbb{R}^{2}: x_{1}^{2}+x_{2}^{2}=r^{2}\right\}
$$

must also be closed.

Example 3.2 (Zariski Topologies of Algebraic Geometry). Let $K$ be a field and let $\mathbb{A}^{n}(K)=K^{n}$. We call $\mathbb{A}^{n}(K)$ affine $n$-space over the field $K$. We can endow $\mathbb{A}^{n}(K)$ with the affine Zariski topology by letting the its closed sets $\mathcal{C}_{n}$ be the polynomial equations in $n$-variables with parameters from $K$ (or, affine varieties, as we called them in our example of definable sets).

Remark 3.3. We will often refer to a topological structure $(M, \mathcal{C})$ by its domain $M$, and we will usually assume that $\mathcal{C}$ is assumed from context.

With these axioms, it is easy to verify some basic facts about topological structures. First, let us note that every projection map $\pi: M^{n+k} \rightarrow M^{n}$ is continuous in 
this topological structure. This is because for each $S \subseteq M^{n}$ closed, the preimage is given by $\pi^{-1}(S)=S \times M^{k}$. By (L3) and (L6), we know that this set is also closed.

In the the topological structure setting, we can identify sets by the formulas which define them. For example, let us consider $S_{1}, S_{2} \in \mathcal{C}$. Then we can define the Boolean operations of $S_{1}$ and $S_{2}$ as such

$$
S_{1}(\bar{x}) \wedge S_{2}(\bar{x})=S_{1} \cap S_{2} \quad S_{1}(\bar{x}) \vee S_{2}(\bar{x})=S_{1} \cup S_{2}
$$

Our only note is that the complement operation nor our quantifiers will necessarily give us a closed sets

Definition 3.4 (Constructible Sets). Let $(M, \mathcal{C})$ be a topological structure with closed sets $\mathcal{C}$. A subset $S \subseteq M^{n}$ is called a constructible set in $M$ if $S$ is equal to a Boolean combination of elements of $\mathcal{C}$. Equivalently, $M$ is constructible if it is a finite union of sets of the form $F \subseteq_{c l} G \subseteq_{o p} M$.

Constructible sets give us a way of expanding our sets of consideration to combinations of sets which may use negation symbols on closed sets. In particular, we can define the closure $\bar{Q}$ as the smallest closed set containing $Q$, and every constuctible set $Q$ can be written in the form

$$
\bigcup_{i=1}^{n} F_{i} \backslash S_{i}
$$

where each $F_{i}$ and $S_{i}$ is closed.

We will now introduce the notion of a projective set to expand our consideration to combinations which might also use the existential quantifiers on some variables in our constructible sets. 
Definition 3.5 (Projective Sets). Let $M$ be a topological structure. A set $S$ is called a projective set in $M$ if $S$ is the image of a constructible set under a projection map. Equivalently, $S$ is projective if it is a finite union of projections of sets of the form $F \subseteq_{c l} G \subseteq_{o p} M$

With the following definitions in place, let us briefly note that every constructible set of $M$ (under a given system of definable closed sets) is a projective set of $M$, and every projective set of $M$ is a definable set of $M$. Often times we will talk about whether or not we can reduce certain classes of subsets to each other, and that will generally denote some kind of topological property.

We now list some extra properties which can be developed for topological structures, which are lifted precisely from definitions sometimes used in algebraic geometry.

Definition 3.6 (Some Topological Properties). The following properties can be used to describe topological structure $M$ (or one of its closed subsets) which satisfies the respective properties :

1. (Complete) : $M$ is called complete if it satisfies the properness of projections property, which we will abbreviate as $(P)$ :

(P) : Let $S \subseteq_{c l} M^{n}$ and pr $: M^{n} \rightarrow M^{m}$ be a projection map. Then $\operatorname{pr}(S) \subseteq_{c l} M^{m}$.

2. (Quasi-Compact) : $M$ is called (quasi-)compact if it is complete and satisfies the following property, which we will abbreviate (QC) :

(QC) : Let $\mathcal{F}=\left\{F_{i}\right\}_{i \in I}$ be a famiy of closed subsets of $M^{n}$. If $\mathcal{F}$ is finitely consistent, then the intersection $\bigcap_{i \in I} F_{i}$ is nonempty. 
3. (Noetherian) : $M$ is called Noetherian if it satisfies the descdending chain condition for its closed subsets. This is the following property, abbreviated $(\mathrm{DCC}):$

(DCC) : Let $S_{i} \in \mathcal{C}$ for $i \in \mathbb{Z}^{+}$be a family of closed sets with

$$
S_{1} \supseteq S_{2} \supseteq \ldots \supseteq S_{n} \supseteq \ldots
$$

then there is some $N \in \mathbb{Z}^{+}$such that $S_{N}=S_{n}$ for all $n \geq N$

The definition of completeness in the above definition comes from the definition of completeness of an algebraic variety. The term quasi-compact was a separate term introduced in algebraic geometry to delineate this definition (which is simply compactness in general topology) from the algebro-geometric notion sometimes used to mean compactness, which means compact and Hausdorff in general topology terms. The Noetherian property is an important property in algebraic geometry, and here we presented the general topological definition of Noetherian.

Definition 3.7 (Irreducible Set). A definable set $S$ is called irreducible if there is no two relatively closed proper subsets $S_{1} \subset_{c l} S$ and $S_{2} \subset_{c l} S$ such that $S=S_{1} \cup S_{2}$.

This definition of an irreducible set is once again a lift of precisely the same notion from algebraic geometry to a general topology context.

Proposition 3.8 (Irreducible Decomposition). Let $S$ be a Noetherian definable subset of a topological structure $M$. Then there is a unique collection of distinct subsets $S_{1}, S_{2}, \ldots, S_{n}$ such that each $S_{i}$ is an irreducible, closed subset of $S$ and $S=S_{1} \cup S_{2} \cup$ $\ldots \cup S_{n}$. Furthermore, each $S_{i}$ is called an irreducible component of $S$. 
Note that if $M$ is Noetherian, then every closed subset is a Noetherian, definable subset of $M$. With that, can expand our decomposition of constructible sets into relatively closed irreducible subsets of open sets in $M$. In other words, if $Q$ is a constructible set of a Noetherian topological space, then we can write

$$
Q=\bigcup_{i=1}^{n} F_{i} \backslash S_{i}
$$

for some $F_{i}, S_{i}$ closed with $F_{i}$ irreducible in $M$.

Let us now list a collection of topological properties which will be useful to note about topological structures.

Theorem 3.9 (Properties of Topological Structures). Let $M$ be a topological structure.

(1.) For $S \subseteq_{c l} M^{n}$ and $\mathrm{pr}: M^{n} \rightarrow M^{k}$, then $\overline{\operatorname{pr}(S)}$ is irreducible if and only if $S$ is irreducible.

(2.) For $n, k \in \mathbb{Z}$, the topology on $M^{n+k}$ extends the product topology on $M^{n} \times M^{k}$.

(3.) If $S_{1}, S_{2}$ are closed, irreducible sets, then $\operatorname{pr}\left(S_{1}\right)$ and $S_{1} \times S_{2}$ are irreducible as well.

(4.) If $F(\bar{x}, \bar{y})$ is a relation defining a closed set, then $\forall \bar{y} F(\bar{x}, \bar{y})$ defines a closed set as well.

Proof. Let us first prove (1). Note that topological closure and images of functions distribute over finite unions, so if $S$ is reducible, the closures of the images of the components of $S$ will serve to demonstrate $\overline{\operatorname{pr}(S)}$ is reducible. Also suppose that 
$\overline{\operatorname{pr}(S)}$ is reducible. Then $\overline{\operatorname{pr}(S)}=F_{1} \cup F_{2}$ for some disjoint closed subsets $F_{1}$ and $F_{2}$. So

$$
\operatorname{pr}^{-1}(\overline{\operatorname{pr}(S)})=\operatorname{pr}^{-1}\left(F_{1} \cup F_{2}\right)=\operatorname{pr}^{-1}\left(F_{1}\right) \cup \operatorname{pr}^{-1}\left(F_{2}\right) \supseteq S
$$

therefore $S_{1}=\operatorname{pr}^{-1}\left(F_{1}\right)$ and $S_{2}=\operatorname{pr}^{-1}\left(F_{2}\right) \cap S$ will form a reduction of $S$. This proves (1).

(3) is fairly easy to prove via similar techniques. (2) follows from the fact that the product topology is the initial topology with respect to projection maps, and so because $M^{n+k}$ continuously maps to each of its factors by the projection map, it must be at least as refined a topology as the product topology on $M^{n+k}$. (4) follows from the fact that

$$
D=\left\{\bar{x} \in M^{n}: \forall \bar{y} F(\bar{x}, \bar{y})\right\}=\bigcap_{\bar{a} \in M^{k}} F\left(M^{n}, \bar{a}\right)
$$

which is closed by the fact that eacy $F\left(M^{n}, \bar{a}\right)$ is closed by axiom (L8), making their intersection also closed.

With some basic topological details in place, we would now like to develop a notion of infinitesimals for a topological structure. The notion of an infinitesimal of a point, say $a \in M$, will essentially be understood as a point that would sit in the closure of $a$ if it existed in the space $M$. This notion will be captured in the following definition, which can be considered a way of expressing a space of infinitesmials.

Definition 3.10 (Specialization). Let ${ }^{*} M \geqslant M$ and let $M \subseteq A \subseteq{ }^{*} M$. A (partial) specialization from $A$ to $M$ is a function $\pi: A \rightarrow M$ such that for each $S$ closed $n$-ary relation defined over $M$ and each $\bar{a} \in M^{n}$, if $\bar{a} \in{ }^{*} S=\left\{\bar{x} \in{ }^{*} M^{n}:{ }^{*} M \vDash S(\bar{x})\right\}$ then $\pi(\bar{a}) \in S$. If $A={ }^{*} M$, then $\pi$ is called a total specialization. 
Example 3.11 (Hyperreals). Let $\mathbb{R}$ and ${ }^{*} \mathbb{R}$ be the real numbers and hyperreals numbers, respectively with their topological structures induced by the positive atomic formulas in the language of ordered fields (the subalgebraic sets over these fields). Consider the subset $\mathbb{R} \subseteq * \mathbb{R}$ defined by

$$
\mathbb{R}=\left\{x \in{ }^{*} \mathbb{R}: x=r+\epsilon \text { for some } r \in \mathbb{R} \text { and } \epsilon>0 \text { such that } \epsilon<s \text { for all } s \in \mathbb{R}\right\}
$$

This set ' $\mathbb{R}$ is often called the set of finite hyperreals. On the finite hyperreals, we can define the standard part map

$$
\text { st }: \mathbb{R} \rightarrow \mathbb{R} \text { by } \operatorname{st}(r+\epsilon)=r
$$

The standard part map is a classical example of a partial specialization from ' $\mathbb{R}$ to $\mathbb{R}$. This can be shown to be a specialization by first showing that the standard part map preserves addition, proving that every set is closed if and only if it is a translate of a closed set about 0 , and then proving that every infinitesimal preserves $M$-closed sets when mapping to 0 . Specialization follows trivially when this fact has been established.

With this example in mind, we realize that we can try to lift some of the same intuitions about specializations from the hyperreals to the general case of specializations for topological structures. The next definition we will establish will be an analogue of the monad (or halo) of a point from nonstandard analysis - that is, we can take the preimage of a point in our base space to obtain its infinitesimal neighborhood with respect to the specialization.

Definition 3.12 (Infinitesimal Neighborhood). Let $\bar{a} \in M$ and (let $\left({ }^{*} M, \pi\right)$ be a 
universal pair for now), then the infinitesimal neighborhood of $\bar{a}$ under $\pi$ is the preimage

$$
\mathcal{V}_{\bar{a}}=\pi^{-1}(\bar{a})
$$

Along with the notion of a specialization, we might find ourselves particularly interested in total specializations since they use the entire structure of the extension, since this would allow us to transfer the same operations and relationships from the base structure to the infinitesimals of the space. The following theorem tells us that if our space is quasi-compact, then we can always guarantee that we can always build such an extension.

Proposition 3.13 (Total Extensions of Partial Specializations). Let $M$ be a quasicompact topological structure and let ${ }^{*} M \geqslant M$. Then any partial specialization from * $M$ to $M$ can be extended to a total specialization. In particular, a total specialization from * $M$ to $M$ exists.

Proof. Let $\pi$ be a partial specialization of ${ }^{*} M$ onto $M$. Suppose $\pi$ is defined on $D \subseteq{ }^{*} M$ and $b^{\prime}$ is an element of ${ }^{*} M$ not in the domain of $\pi$. We want to extend $\pi$ to $D \cup\left\{b^{\prime}\right\}$. Let

$$
\Phi=\left\{S(x, \bar{d}): S \subseteq_{c l} M^{n+1} \text { and }{ }^{*} M \vDash S\left(b^{\prime}, \bar{d}^{\prime}\right) \text { for some } \bar{d}^{\prime} \in D^{n} \text { where } \pi\left(\bar{d}^{\prime}\right)=\bar{d}\right\}
$$

By definition, each of these $S(M, \bar{d})$ sets is empty because $\exists x S\left(x,{ }^{*} M\right)$ is nonempty by definition, and is closed by completeness of quasi-compact spaces, so $M \vDash \exists x S\left(x, \pi\left(d^{\prime}\right)\right)$. Furthermore, every finite intersection of such sets is in the set, so they must also be nonempty. Therefore, by quasi-compactness, the whole intersection of sets defined by formulas in $\Phi$ is nonempty. Let $b \in \bigcap \Phi(M)$ and extend $\pi\left(b^{\prime}\right)=b$. This extension is 
a partial specialization. We can then proceed to do this inductively for each $b \in{ }^{*} M$ until $\pi$ is total.

To prove that a total specialization will always exist, note that the identity map is a partial specialization that always exists - and by the preceding argument, it has a total extension.

We would like to understand what kind of topological information a specialization captures about the structure onto which it specializes. We can define a topological structure by considering the $\pi$-closed subsets of $M$ induced by the specialization of ${ }^{*} M$ onto $M$.

Definition 3.14 ( $\pi$-closed Sets). Let $\pi:{ }^{*} M \rightarrow M$ be a partial specialization. We say a definable set $P \subseteq M^{n}$ is $\pi$-closed if $\pi\left({ }^{*} P\right) \subseteq P$. The collection of such sets is denoted $\mathcal{C}_{\pi}$ for specialization $\pi$.

In context of our definition of specializations, it should be noted that every closed set (element of $\mathcal{C}$ ) is automatically closed, however this family of closed sets does not encompass all definable subsets - as we've already observed with our open subsets, which are definable over $M$, but not necessarily closed. We also note that $P \subseteq \pi\left({ }^{*} P\right)$ follows for free since $P \subseteq{ }^{*} P$ and $\pi(\bar{p})=\bar{p}$ for all $\bar{p} \in P$. This means we could have replaced ' $\subseteq$ ' with '=' in the definition to obtain an equivalent definition.

We would like to note some of the topological properties that the topologies of these

Proposition 3.15 (Saturated Total Specializations demonstrate Compactness). Let $\pi:{ }^{*} M \rightarrow M$ be a total specialization with ${ }^{*} M$ saturated. Then the topological structure of $\pi$-closed subsets of $M$ is quasi-compact. 
Proof. Completeness is immediate, since every map commutes with projection and projections of closed sets are definable.

To prove quasi-compactness, consider a family of closed sets $\left\{S_{i}\right\}_{i \in I}$ in $M^{n}$. Then $\bigcap_{i \in I}{ }^{*} S_{i}$ is nonempty since ${ }^{*} M$ is saturated. Let $c^{\prime}$ be in the intersection in ${ }^{*} M$. Then $\pi\left(c^{\prime}\right) \in \bigcap_{i \in I} S_{i}$. Therefore $M$ is quasi-compact.

One note about the proof of this theorem is that our proof that $\mathcal{C}_{\pi}$ never invoked the saturation of ${ }^{*} M$, nor did it invoke the totality of the specialization. This means we have also proven that the topological structure induced by $\mathcal{C}_{\pi}$ is always complete. Furthermore, we realize that our choice of specialization onto our structure $M$ will induce different properties on the structure of $\pi$-closed sets. With this in mind, we motivate the next definition with the aim of identifying a class of specializations onto $M$ under which the $\pi$-closed sets and the ordinary closed sets of $M$ coincide.

Definition 3.16 (Universal Specialization). Let ${ }^{*} M \geqslant M$ and let $\pi:{ }^{*} M \rightarrow M$ be a specialization. We call this pair $\left({ }^{*} M, \pi\right)$ a universal pair over $M$ if for every elementary extension ' $M \geqslant{ }^{*} M$ and any finite $A \subseteq M$ and specialization $\pi^{\prime}: A \cup{ }^{*} M \rightarrow$ $M$ extending $\pi$, there is an elementary embedding $\alpha: A \rightarrow{ }^{*} M$ over $A \cap{ }^{*} M$ such that

$$
\pi^{\prime}=\pi \circ \alpha \text { over } A
$$

Proposition 3.17 ( $\pi$-closed sets are precisely closed sets in Universal Specializations). Let $\left({ }^{*} M, \pi\right)$ be a universal pair over $M$, and let $S \subseteq M^{n}$ be a definable set of M. Then $S$ is $\pi$-closed if and only if $S$ is closed. In other words, $\mathcal{C}=\mathcal{C}_{\pi}$.

Proof. We start by proving the following claim 
Claim 3.18. Let $S_{0}$ be a relatively closed subset of $S$, and suppose that for some $s \in S$ there is a $s^{\prime} \in \mathcal{V}_{s} \cap S_{0}$. Then $s \in S_{0}$.

This claim follows fairly trivially from the fact that $S_{0}=\overline{S_{0}} \cap S$ and $s=\pi\left(s^{\prime}\right) \in \overline{S_{0}}$ by $s^{\prime} \in \mathcal{V}_{s}$. This gives us that every closed subset is $\pi$-closed. Now let us prove the other direction.

The other direction is proven in [14] as Proposition 2.2.24 in Chapter 2, Section 2.

With this in mind, we would like to work with universal specializations whenever we can, so that our infinitesimals faithfully represent the closed subsets of our base space. Fortunately, we can always guarantee the existence of such a universal specialization.

Proposition 3.19 (Universal Specialization Existence). Every topological structure $M$ has a universal pair $\left({ }^{*} M, \pi\right)$. Furthermore, if $M$ is quasi-compact, this $\pi$ will be total.

Proof. The proof here will replicate the general kind of proof strategy used to build saturated extensions.

Begin with $M_{0} \geqslant M$ with specialization $\pi_{0}: M_{0} \rightarrow M$. We construct a chain of elementary extensions $M_{0} \leqslant M_{1} \leqslant \ldots \leqslant M_{i} \leqslant \ldots$ of length $\omega$ with partial specialization $\pi_{i}: M_{i} \rightarrow M$ and $\pi_{i} \subseteq \pi_{i+1}$ for each $i<\omega$. In the case where $M$ is quasi-compact, we note that we can always choose $\pi_{i}$ to be total.

To construct each pair $\left(M_{i+1}, \pi_{i+1}\right)$ given $\left(M_{i}, \pi_{i}\right)$ constructed, consider the set

$$
\left\{\left(A_{\alpha}, \bar{a}_{\alpha}, p_{\alpha}(\bar{x})\right): \alpha<\kappa_{i}\right\}
$$


where each $A_{\alpha}$ is a finite subset of $M_{i}, \bar{a}_{\alpha} \in M^{n}$, and $p_{\alpha}(\bar{x})$ is an $n$-type over $M \cup A_{\alpha}$ and $\kappa$ is the cardinality of all possible tuples. We now construct specializations $\pi_{i, \alpha}$ for each $\alpha<\kappa_{i}$ such that $\pi_{i, \alpha} \supset \pi_{i}$ with domain extended to some $N_{i, \alpha}$.

Let $N_{i, 0}=M_{i}$ and $\pi_{i, 0}=\pi_{i}$. At the limit ordinal steps $\alpha$, take the union of all functions defined at previous steps. On successor steps, follow the decision rule

(i.) If there is some $\bar{b}$ satisfying $p_{\alpha}$, and a specialization $\pi^{\prime} \supseteq \pi_{i, \alpha}$ sending $\bar{b}$ to $\bar{a}_{\alpha}$, then let $N_{i, \alpha+1}=N_{i, \alpha} \cup\{\bar{b}\}$ and $\pi_{i, \alpha+1}=\pi^{\prime}$

(ii.) Otherwise, let $N_{i, \alpha+1}=N_{i, \alpha}$ and let $\pi_{i, \alpha+1}=\pi_{i, \alpha}$

Now let $M_{i+1}$ be a model containing $N_{i, \kappa_{i}}$ and let $\pi_{i+1}$ be a specialization extending $\pi_{i, \kappa_{i}}$ from $M_{i+1}$ to $M$. Again, if $M$ is quasi-compact, these can be chosen to be total.

We finally note that for each ' $M \geqslant M_{i+1} \geqslant M$ and any finite subset $B \subseteq ' M$ with specialization $\pi^{\prime}: B \cup M_{i} \rightarrow M$ extending $\pi_{i} \mathrm{~m}$ we can define an elementary isomorphism (that is, a bijective map that preserves first-order formulas in both directions) $\alpha: B \rightarrow M_{i+1}$ over $M \cup\left(B \cap M_{i}\right)$ such that $\pi^{\prime}=\pi \circ \alpha$ over $B$. Therefore, if we let

$$
{ }^{*} M=\bigcup_{i<\omega} M_{i} \text { and } \pi=\bigcup_{i<\omega} \pi_{i}
$$

then we will have $\left({ }^{*} M, \pi\right)$ as a universal pair.

As noted in the proof of the previous theorem, let us also note the following, which can be obtained by a modification of the proof of the previous theorem, but making sure to alternate between steps that saturate the structure and steps that build a universal specialization on the structure. 
Proposition 3.20 (Universal and Saturated Extension). Let $M$ be a topological structure with universal pair $\left({ }^{*} M, \pi\right)$ specializing to it. Then there is a structure ${ }^{*} M \geqslant{ }^{*} M$ and a specialization ${ }^{*} \pi:{ }^{* *} M \rightarrow{ }^{*} M$ such that the following hold:

(1.) $\left({ }^{* *} M,{ }^{*} \pi\right)$ is a universal pair over ${ }^{*} M$.

(2.) $\left({ }^{*} M, \pi \circ{ }^{*} \pi\right)$ is a universal pair over $M$.

(3.) ${ }^{* *} M$ is $\left|{ }^{*} M\right|^{+}$-saturated.

In other words, every topological structure has a saturated elementary extension which universally specializes to it.

With this in place, we can now start developing more geometrical notions for such structures, such as dimension and covering maps. In particular, we will consider certain kinds of topological structures with particularly nice dimension notions and projection properties Zariski structures, which are an intermediate topological structure towards building Zariski geometries, which have many of the nice modeltheoretical propreties of Zariski geometries, but lack some of the nice properties which make the geometry on these structures similar to algebraic varieties over algebraically closed fields.

\subsection{Zariski Structures}

With the basic topological structure notions in place, we would like to start introducing some more geometrical structure to these topologies, and begin to develop some commonly used constructs that often appear in more geometrically-oriented studies of topological spaces. In particular, we will introduce the notion of a Zariski structures. The geometrical conditions of Zariski structures will be shown to admit 
nice model-theoretical properties and transfer under taking elementary extensions of Zariski structues - particularly the universal specializations over the space. We will also introduce the notion of covering maps of topological structures, which will be useful later when we develop infinitesimal analysis over a more well-behaved subclass of Zariski structures, which will be our Zariski geometries. We will begin by introducing a good dimension notion for topological spaces.

Definition 3.21 (Good Dimension Notion). Let $X$ be the family of all projective subsets of $M$. A function $\operatorname{dim}: X \rightarrow \omega$ is called a good dimension notion if it satisfies the following properties :

-(DP) Dimension of Points : $\operatorname{dim}(\{\bar{a}\})=0$ for every $\bar{a} \in M^{n}$. Every point is 0dimensional.

-(DU) Dimension of Unions : $\operatorname{dim}\left(S_{1} \cup S_{2}\right)=\max \left\{\operatorname{dim}\left(S_{1}\right), \operatorname{dim}\left(S_{2}\right)\right\}$ for every $S_{1}, S_{2} \in$ $X$.

-(SI) Strong Irreducibility : Let $S \subseteq_{c l} U \subseteq_{o p} M^{n}$ and let $S$ be irreducible. For any relatively closed subset $S^{\prime} \subseteq_{c l} S$ where $S^{\prime} \neq S$ we have $\operatorname{dim}\left(S^{\prime}\right)<\operatorname{dim}(S)$.

-(AF) Addition Formula : Consider an irreducible $S \subseteq_{c l} U \subseteq_{o p} M^{n}$ and projection pr : $M^{n} \rightarrow M^{m}$. Then

$$
\operatorname{dim}(S)=\operatorname{dim}(\operatorname{pr}(S))+\min _{\bar{a} \in \operatorname{pr}(S)}\left\{\operatorname{dim}\left(\operatorname{pr}^{-1}(\bar{a})\right) \cap S\right\}
$$

-(FC) Fiber Condition : For any irreducible $S \subseteq_{c l} U \subseteq_{o p} M^{n}$ and projection pr : $M^{n} \rightarrow$ $M^{m}$. Then there is a relatively open subset $V \subseteq_{o p} \operatorname{pr}(S)$ such that for any $\bar{b} \in V$

$$
\min _{\bar{a} \in \operatorname{pr}(S)} \operatorname{dim}\left(\operatorname{pr}^{-1}(\bar{a}) \cap S\right)=\operatorname{dim}\left(\operatorname{pr}^{-1}(\bar{b}) \cap S\right)
$$


In other words, there is an open subset on which the dimension of the fibers of $\operatorname{pr}(S)$ is minimized.

This notion of dimension is defined to mimic the kinds of dimension properties that we find with dimension notions from algebraic geometry, such as the dimension of an irreducible variety in terms of the maximal lengths of descending chains of irreducible subvarieties. Furthermore, with this notion of dimension, we note that we can now give a notion of a what it means for a point to be generic, which will be a convenient notion for proving generic properties of subsets of topological structures.

Definition 3.22 (Generic Point). For $M<^{'} M$, we say that a point $\bar{a}^{\prime} \in{ }^{\prime} M^{n}$ and let $S \subseteq_{c l} M^{n}$ be an irreducible subset such that $\bar{a}^{\prime} \in S\left({ }^{\prime} M\right)$. If $\operatorname{dim}(S)$ is minimal with respect to all closed subsets satisfying these conditions, then we say that $\bar{a}^{\prime}$ is generic in $S$.

To clarify this definition of a generic point, consider the following interpretation of what it means for $\bar{a}^{\prime} \in S\left({ }^{\prime} M\right)$ to be non-generic : this means that $\bar{a}^{\prime}$ is in an irreducible, closed subset of $\hat{S} \subseteq M^{n}$ with $\operatorname{dim}(\hat{S})<\operatorname{dim}(S)$. Therefore $\hat{S} \cap S$ must have strictly smaller dimension than $S$, which means that we can omit $\hat{S}$ from $S$ to obtain a subset $S \backslash \hat{S}$ which keeps the same dimension, but now omits the point $\bar{a}^{\prime}$ in the specialization. In other words, the set of generic points of $S$ are the set of points from $S$ that cannot be removed without also removing a non-negligible component of $S$ and its infinitesimals.

Definition 3.23 (Zariski Structure). Let $M$ be a Noetherian topological structure. If $M$ is endowed with a good dimension notion $\operatorname{dim}$, then $(M, \operatorname{dim})$ - often denoted as simply $M$ - is called a Zariski structure if it satisfies the following condition : 
-(SP) Semi-properness of projections : For any closed irreducible subset $S \subseteq_{c l} M^{n}$ and projection $\operatorname{pr}: M^{n} \rightarrow M^{m}$, there is a subset $F \subset_{c l} \overline{\operatorname{pr}(S)}$ closed in $M^{m}$ such that $\overline{\operatorname{pr}(S)} \backslash F \subseteq \operatorname{pr}(S)$

Recall that the Noetherian property of a Zariski structure $M$ means that every closed subset of $M$ is reducible to a finite union of irreducible components. With this in mind, one can often prove theorems without loss of generality on irreducible subsets. Thus we can assume that $M$ itself is irreducible without loss of generality. Let's also note the following propositon, which will be useful later when we proving some facts about coverings of Zariski structures. We present it without proof.

Proposition 3.24 ((Addition Formula for Reducible Sets)). For a topological structure $M$ with a good dimension notion, assume $S \subseteq_{c l} U \subseteq_{o p} M^{n}$ and that $S_{0} \subseteq_{c l} S$ with $\operatorname{dim}\left(S_{0}\right)=\operatorname{dim}(S)$. Then

$$
\operatorname{dim}(S) \geq \operatorname{dim}(\operatorname{pr}(S))+\min _{\bar{a} \in \operatorname{pr}(S)}\left\{\operatorname{dim}\left(\operatorname{pr}^{-1}(\bar{a}) \cap S\right)\right\}
$$

Along with the usual properties of Zariski structure, we also list the following properties which Zariski structures can have; which we be useful later when we develop the notion of a so-called Zariski geometry.

Definition 3.25 (Some Additional Properties for Zariski Structures). Let $M$ be a topological structure. Here we define the following properties, labelled by the abbreviations we will use for the properties :

-(EU) Essential Uncountability : We say $M$ is essentially uncountable if for every $S \subseteq_{c l} M^{n}$ such that $S=\bigcup_{k=0}^{\infty} S_{k}$ for some $S_{k} \subseteq_{c l} M^{n}$, then there is finitely-many of those sets $S_{k_{1}}, S_{k_{2}}, \ldots, S_{k_{n}}$ such that $S=\bigcup_{i=1}^{n} S_{k_{i}}$. 
-(PS) Pre-Smoothness : We say $M$ is pre-smooth if for every $S_{1}, S_{2} \subseteq_{c l} M^{n}$ irreducible subsets, then for every $S^{\prime} \subseteq_{c l} S_{1} \cap S_{2}$ irreducible component, we have

$$
\operatorname{dim}\left(S^{\prime}\right) \geq \operatorname{dim}\left(S_{1}\right)+\operatorname{dim}\left(S_{2}\right)-\operatorname{dim}\left(M^{n}\right)
$$

We will occasionally note some results about these properties along the way to defining Zariski geometries, but for now we will consider them imporant for what model theoretic results they will admit in structures which satisfy them. In particular, the next theorem will give us one of the main model-theoretical properties about Zariski structures, which will demonstrate how powerful the logical relationships on these structures are, and give us directions for how to adapt these structures to become minimal structures in the sense described in chapter 2 .

Theorem 3.26 (Zariski Structures Admit Quantifer Elimination). Let $M$ be a Zariski strucrure. Then for every definable subset $Q \subseteq M^{n}, Q$ is constructible.

Proof. Recall that any constuctible set is of the form

$$
Q=\bigcup_{i=1}^{n} S_{i} \backslash P_{i}
$$

where each $S_{i}, P_{i}$ is closed and irreducible. Consider the projection map pr: $M^{n+1} \rightarrow$ $M^{n}$. It is sufficient to prove that $\operatorname{pr}(Q)$ will be constuctible itself. Without loss of generality, we may also assume $Q=S \backslash P$ with $Q$ nonempty. Let us proceed by induction on $\operatorname{dim}(S)$. Let

$$
d_{S}=\min _{\bar{a} \in \operatorname{pr}(S)}\left\{\operatorname{dim}\left(\operatorname{pr}^{-1}(\bar{a}) \cap S\right)\right\}=\min \{\operatorname{dim}(S(M, \bar{a})): \bar{a} \in \operatorname{pr}(S)\}
$$


and let

$$
F=\left\{\bar{b} \in \operatorname{pr}(S): \operatorname{dim}(P(M, \bar{b})) \geq d_{S}\right\}
$$

We note that the closure of $F$ is $\bar{F} \subset \overline{\operatorname{pr}(S)}$ by $(\mathrm{FC})$. Thus $\operatorname{dim}(\bar{F})<\operatorname{dim}(\overline{\operatorname{pr}(S)})$ since $\overline{\operatorname{pr}(S)}$ is irreducible. Then we let $S^{\prime}=S \cap \operatorname{pr}^{-1}(\bar{F})$. From this, we gather than $S^{\prime} \subset S$ since $\bar{F} \cap \operatorname{pr}(S) \neq \operatorname{pr}(S)$. So $\operatorname{dim}\left(S^{\prime}\right)<\operatorname{dim}(S)$ by irreducibility as well. Then finally, we see

$$
\operatorname{pr}(Q)=\operatorname{pr}(S \backslash P) \subseteq \operatorname{pr}\left(S^{\prime} \backslash P\right) \cup(\operatorname{pr}(S) \backslash F)
$$

But $\operatorname{pr}(S) \backslash F \subseteq \operatorname{pr}(Q)$ since if $\bar{b} \in \operatorname{pr}(S) \backslash F$, then $P(M, \bar{b}) \subset S(M \bar{b})$ This makes the equality hold

$$
\operatorname{pr}(Q)=\operatorname{pr}\left(S^{\prime} \backslash P\right) \cup(\operatorname{pr}(S) \backslash F)
$$

We then apply induction on $S^{\prime} \backslash P$ and note that $\operatorname{pr}(S) \backslash F$ is already constructible.

Given that we can do quantifier elimination in a Zariski structure, this means that we can extend our definition of dimension to all definable subsets since every $Q$ definable in $M$ can be written as

$$
Q=\bigcup_{i=1}^{n}\left(S_{i} \backslash P_{i}\right)
$$

with each $S_{i}, P_{i}$ closed and each $S_{i}$ irreducible since $M$ is Noetherian. This means that we can take the closure to be

$$
\bar{Q}=\bigcup_{i=1}^{n} S_{i}
$$

which means that we can compute $\operatorname{dim}(Q)=\operatorname{dim}(Q)=\max _{1 \leq i \leq n}\left\{\operatorname{dim}\left(S_{i}\right)\right\}$. Furthermore, in a Zariski structure we can work with the following additional form of the Fiber 
condition.

Proposition 3.27. Let $M$ be a Zariski structure. Then the following condition holds

- $\left(F C^{\prime}\right)$ For any projection map pr, closed irreducible $S \subseteq M^{n}$, and $k \in \mathbb{N}$; the set

$$
\mathcal{P}^{\operatorname{pr}}(S, k)=\left\{a \in \operatorname{pr}(S): \operatorname{dim}\left(S \cap \operatorname{pr}^{-1}(a)\right)>k\right\}
$$

is constructible and is contained in a proper, relatively closed subset of $\operatorname{pr}(S)$ provided

$$
k \geq \min _{a \in \operatorname{pr}(S)}\left\{\operatorname{dim}\left(\operatorname{pr}^{-1}(a) \cap S\right)\right\}
$$

Outline. Proven by induction on dimension of $S$. The statement is trivial for zerodimensional case, and the case where $k<\min _{\bar{a} \in \operatorname{pr}(S)}\left\{\operatorname{dim}\left(\operatorname{pr}^{-1}(\bar{a}) \cap S\right\}\right.$. We can substract the union of the fibers over the open set obtained from (FC) from the set $S$ to obtain a subset $S^{\prime}$ of smaller dimension, which we can show that $\mathcal{P} \operatorname{pr}(S, k)=$ $\mathcal{P}^{\operatorname{pr}}\left(S^{\prime}, k\right)$, where we can also show $S^{\prime}$ is contained in $\operatorname{pr}(S) \backslash U$.

We would now like to prove some results about the Morley rank of Zariski structures. In particular, given our motivation, we should be expecting that some general classes of Zariski structures will have at least finite Morley rank, as we can see with irreducible varieties over algebraically closed fields - since their dimensions are equal to their Morley ranks. In fact, essential uncountability constitutes one of these classes of structures. This can be captured in the following model-theoretical reformulation of essential uncountability, which is proven in [14].

Lemma 3.28. A Zariski structure $M$ is essentially uncountable if and only if it is $\omega_{1}$-compact, ie all of its countable types are realized. 
As a brief remark, we note that $\omega_{1}$-compactness is not $\omega_{1}$-saturation, since a type can include uncountably-many sentences using countably-many parameters. However, we can note that every $\omega_{1}$-saturated structure must be $\omega_{1}$-compact. With this lemma, we can prove that essentially uncountable Zariski structures have finite Morley rank fairly easily.

Theorem 3.29 (Essentially Uncountable Zariski Structures have Finite Morley Rank). Any Zariski structure $M$ satisfying (EU) has finite Morley rank. More precisely, $\operatorname{rk}(Q) \leq \operatorname{dim}(Q)$ for any definable set $Q$ of $M$.

Proof. We first note that if $\operatorname{dim}(Q)=0$, then $Q$ must be finite. Hence $\operatorname{rk}(Q)=0$. This initialized the base case of an inductive proof. We will now prove the inductive step by contradiction. Suppose that $\operatorname{dim}(Q) \leq n$ but $\operatorname{rk}(Q) \geq n+1$ for some $n \geq 0$. We may assume without loss of generality that $Q$ is irreducible. By definition of Morley rank, we know that there must be, for any $k \in \mathbb{Z}^{+}$, some disjoint $Q_{1}, Q_{2}, \ldots, Q_{k}$ with $\operatorname{rk}\left(Q_{i}\right) \geq n$ such that

$$
Q \supseteq Q_{1} \cup Q_{2} \cup \ldots Q_{i}
$$

Let $i=2$. Then by the induction hypothesis $\operatorname{dim}\left(Q_{1}\right), \operatorname{dim}\left(Q_{2}\right) \geq n$. Since $Q$ is irreducible, $\operatorname{dim}\left(Q_{1} \cap Q_{2}\right) \geq n \geq 0$, which is a contradiction since these sets are disjoint.

This theorem will be useful later when we start to develop some pieces of the dimension theory of Zariski geometries in chapter 4.

Now that we have defined the notion of a Zariski structure, let us consider more properties about algebraic varieties. In particular, let us note the following theorem; which should elucidate some of the connections between these properties and classical algebraic geometry in a more concrete way. 
Theorem 3.30 (Algebraic Varieties over ACFs are Zariski Structures). Let $M$ be an algebraic variety over some $\mathbb{A}^{n}(K)$ for some algebraically closed field $K$ with its induced subspace topological structure from $\mathbb{A}^{n}(K)$, and dimension notion defined as its Krull dimension. Then $M$ is a Zariski structure. Furthermore $M$ satisfies (PS) if $M$ is smooth, and $M$ satisfies (EU) if and only if $K$ is uncountable.

Outline and References. Verifying that varieties are Noetherian topological structures is a fairly standard set of properties proven in standard textbooks in algebraic geometry. As we also noted, the given definition of dimension can be easily shown to satisfy (DP), (DU), and (SI) in the definition of a good dimension notion. The proof of (AF) and (FC) can be found in [9] as Theorem 1.25 in Chapter 1, Section 6. (PS) is proven using basic arguments about dimensions of tangent spaces and local dimension of varieties, which can be found in Chapter 2 of [9].

We will remark in the next chapter that the model-theoretical properties of algebraic varieties are much richer than the model theory of general Zariski structures. We will seek to isolate the properties which capture this amount of model-theoretical characterization in the notion of a Zariski geometry, which will encompass the kinds of structures whose geometries will even more closely ressemble algebraic varieties. Until then, let us turn our attention to some other constructs pertaining to the analysis of Zariski structures which will be useful for us later when we develop such geometries.

\subsection{Elementary Extensions of Zariski Structures}

With some of the general definitions, properties, and examples of Zariski structures provided; we can now go ahead and start to develop some more of the theory of how the properties of Zariski structures are preserved under operations such as taking 
elementary extensions and substructures, so that we can later develop some theory of how to construct new topological and Zariski structures from old ones while still remaining within the same general classes of topological structures. In particular, we can canonically define a Zariski structure on any elementary extension of a Zariski structure.

Construction 3.31 (Zariski Topology of Elementary Extensions). Let ' $M \geqslant M$. We declare any set of the form $S=S\left(\bar{a},{ }^{\prime} M^{m}\right)$ is closed whenever $S$ is a closed subset of $M^{(}(l+m)$ and $\bar{a} \in^{\prime} M^{l}$. If a closed set is defined with parameters from $A$, we call the set $A$-closed.

Furthermore, we can extend the dimension notion of $M$ to ' $M$ by letting

$$
\operatorname{dim}\left(S\left(\bar{a},{ }^{\prime} M\right)\right)=\max \{k \in \mathbb{N}: \bar{a} \in \mathcal{P}(S, k)\}+1
$$

where

$$
\mathcal{P}(S, k)=\mathcal{P}^{\operatorname{pr}}(S, k)=\left\{a \in \operatorname{pr}(S): \operatorname{dim}\left(S \cap \operatorname{pr}^{-1}(\bar{a})\right)>k\right\}
$$

from the augmented fiber condition in Proposition 3.24. This holds because if $S \subseteq$ $M^{\ell+m}$ is $M$-closed, then there is a bound $b=m \operatorname{dim}(M)$ on the dimension of the fibers of $S$ by (AF). So there is a maximal $b \in \mathbb{N}$ such that $\bar{a} \in \mathcal{P}(S, b)$, and since this set is definable, then we know that this set will be satisfied in ' $M$ precisely for each $k$ for which it is satisfied in $M$.

With some extra verification, we can demonstrate that this Zariski structure on ' $M$ is well-defined so long as we assume $M$ is an essentially uncountable Zariski structure. This is captured in Theorem 3.5.25 in Chapter 3, Section 5 of [14]. An important 
tool in studying the elementary extensions of Zariski structures is the following, which is indeed used to prove the well-definedness of this extension.

Definition 3.32 (Combinatorial Dimension). For $\bar{a} \in{ }^{\prime} M^{n}$ and $A \subseteq ' M$, define the locus of $\bar{a}$ over $A$ to be the intersection of all $A$-closed sets containing $\bar{a}$. Then define the combinatorial dimension of $\bar{a}$ over $A$ as

$$
\operatorname{cdim}(\bar{a} / A)=\operatorname{dim}(\operatorname{locus}(\bar{a} / A))
$$

This definition is indeed a generalization of the notion of transcendence degree used in algebraic geometry and field theory. In particular, suppose that ' $K$ is a field extension of an algebraically closed field $K$, and suppose that $\bar{a} \in \mathcal{\prime}^{\prime} K^{n}$ and $A \subseteq ' K$, then

$$
\operatorname{cdim}(\bar{a} / A)=\operatorname{tr} \cdot \mathrm{d}(\bar{a} / A)
$$

In this sense, the function cdim gives a means of defining the algebraic dependence of $\bar{a}$ on the set of objects $A$. The following proposition captures this more precisely, and will be useful later when we discuss elementary extensions of Zariski geometries.

Proposition 3.33 (Loci Minimize dimension of Fibers). Assume $S$ is closed in $M^{l+k}$ and $\mathrm{pr}: M^{l+k} \rightarrow M^{k}$ is the projection map, and assume $\overline{\operatorname{pr}(S)}=\operatorname{locus}(\bar{a} / M)$ for some $\bar{a} \in^{\prime} M^{k}$. Then

$$
\operatorname{dim}\left(S\left(\bar{a},{ }^{\prime} M\right)\right)=\min _{\bar{a}^{\prime} \in \operatorname{pr}(S)}\left\{\operatorname{dim}\left(S\left(\bar{a}^{\prime}, M\right)\right)\right\}
$$

Proof. Let $\ell=\operatorname{dim}\left(S\left(\bar{a},{ }^{\prime} M\right)\right)$. Then $\bar{a} \in \mathcal{P}(S, \ell-1)$, and hence $\mathcal{P}(S, \ell-1)=\operatorname{pr}(S)$.

With some of these basic facts in mind, let us turn towards other tools which are useful for analyzing Zariski structures. In particular, let us note some basic 
results about how topological structure with dimension can be analyzed by irreducible relations over them.

\subsection{Coverings of Zariski Structures}

Another tool useful in algebraic geometry and topology is the notion of covering spaces and branched coverings. To generalize these to the context of topological structures with dimension, we use the following definition, along with some of the properties these covers can have

Definition 3.34 (Coverings and some related definitions). Let $M$ be a topological structure with dimension. If $F(\bar{x}, \bar{y}) \subseteq_{c l} V \subseteq_{o p} M^{n} \times M^{k}$ is irreducible and pr : $M^{n} \times$ $M^{k} \rightarrow M^{n}$ such that $\operatorname{pr}(F)=D$, then we say $F$ is an (irreducible) covering of $D$

- $($ Discrete Covering): The covering is called discrete over $\bar{a} \in D$ if $\operatorname{dim}(F(\bar{a}, \bar{v}))=$ 0.

- (Finite Covering) : The covering is called finite over $\bar{a} \in D$ if $F\left(\bar{a}, M^{k}\right)$ is finite.

- (Dimension of Generic Fiber) : We define $r=\min _{\bar{a} \in D}\left(\operatorname{dim}\left(F\left(\bar{a}, M^{k}\right)\right)\right)$ the dimension of a generic fiber of the cover $F$.

- (Regular Point of a Cover) : We call a point $\bar{a} \in D$ a regular point of $F$ if $\operatorname{dim}(F(\bar{a}, \bar{y}))$ is equal to the dimension of a generic fiber. Let us denote the set of regular points of $F$ over $D$ by $\operatorname{reg}(F / D)$

With branched coverings, the main idea is that we can identify all the points of the base space (the space being covered) which have zero-dimensional fibers - which in the ordinary context corresponds to identifying points over which the branched covering is simply a covering map. The next proposition and its corollaries aim to 
elucidate some of our choices in our previous definition and how they will serve as a nice tool for doing dimension theory on topological structures.

Proposition 3.35 (Bound on Dimension of Irregular Points). Let $F$ be a covering of $D$. Then

$$
\operatorname{dim}(D \backslash \operatorname{reg}(F / D)) \leq \operatorname{dim}(D)-2
$$

Proof. Let the set of points in $F$ over irregular points $F^{\prime}=\{(a, b) \in F: a \in(D \backslash \operatorname{reg}(F / D))\}$ is a proper closed subset of $F$. But $(\mathrm{SI})$ we know $\operatorname{dim}\left(F^{\prime}\right)<\operatorname{dim}(F)$. By the addition formula for reducible sets in Proposition 3.24,

$$
\operatorname{dim}\left(F^{\prime}\right) \geq r+1+\operatorname{dim}(D \backslash \operatorname{reg}(F / D))
$$

The inequality follows from some algebraic manipulation.

This upper bound allows us to isolate some nice cases of coverings. In particular, we get the following case almost trivially

Corollary 3.36 (One-dimensional base spaces are regular). Let $F$ be a covering of an irreducible set $D$ where $\operatorname{dim}(D)=1$. Then every $a \in D$ is regular under the covering.

Also, even without using the proposition, we can recover a case similar to the ordinary case of regular points in the context of branched coverings. This is captured in the next proposition, which is trivial from the definitions.

Proposition 3.37. Let $F$ be a finite cover of $D$ at a. Then the dimension of a generic fiber of $F$ is 0 . Furthermore, for every $a^{\prime} \in \mathcal{V}_{a}$ we have $a^{\prime} \in \operatorname{reg}\left(F^{*} / D^{*}\right)$ where $\left({ }^{*} M, \pi\right)$ is a universal pair with saturated ${ }^{*} M$ over $M$. 
While there are some interesting properties of coverings in the context of general Zariski structures, their true power can be recognized when we study covers over presmooth sets, and what those covering properties will tell us in context of studying the Zariski geomtries we have been anticipating throughout this chapter. In particular, we will recognize that our notion of pre-smoothness is defined purely dimensionally, and so these theorems regarding the dimensions of fibers at points will give us ways to see how pre-smoothness transfers between covering spaces and their base spaces. 


\section{CHAPTER 4}

\section{CLASSICAL ZARISKI GEOMETRIES}

Zariski structures provide an excellent building block towards the kinds of classification theorems we expect to resolve the Zilber trichotomy problem, but we will see in chapter 5 that these axioms are not quite sufficient to complete our classification. Throughout this chapter, we will develop the notion of a Zariski geometry, which will be a special kind of Zariski structure which admits a notion of infinitesimal analysis and a notion of families of curves which have branches with trajectories that can be classified according to their tangency. We will then use these notions to define group and field structures on the curves of sufficiently ample Zariski geometries, which will then allow us to determine that these ample Zariski geometries consist only of curves which can be interpreted in in these field structures that the curves define.

\subsection{Zariski Geometries}

Before we define Zariski geometries, we will need to first generalize the definition of pre-smoothness to all constructible sets. Fortunately, this is mostly just a simple lift of the previous definition of pre-smoothness to the induced subspace topology on the contructible subset, but with some extra condition that guarantee properties pertaining to pre-smoothness transfer well when passing to substructures defined by constructible subsets. 
Definition 4.1 (Pre-smooth set). A constructible set $A$ of $M$ will be called presmooth in $M$ if for any two relatively closed subsets $S_{1}, S_{2} \subseteq_{c l} A^{k} \times M^{m}$ and any irreducible component $S^{\prime} \subseteq_{c l} S_{1} \cap S_{2}$ we have

$$
\operatorname{dim}\left(S^{\prime}\right) \geq \operatorname{dim}\left(S_{1}\right)+\operatorname{dim}\left(S_{2}\right)-\operatorname{dim}\left(A^{k} \times M^{m}\right)
$$

We now define a Zariski geometry as a Zariski structure in which all constructible sets are constructible. Following our analogy, if we could establish affine spaces are Zariski geometries, then that would be equivalent to proving that every set defined by a quantifier-free formula is smooth.

Definition 4.2 (Zariski Geometry). Let $M$ be a Zariski structure. We say that $M$ is a Zariski geometry if it is essentially uncountable (EU) and satisfies the following strong presmoothness property, abbreviated (sPS):

(sPS) A topological structure with dimension $M$ is strongly presmooth if all of its constructible subsets are presmooth in $M$.

Some of the nice properties of Zariski geometries follow from the following lemma that essentially says that fibers of pre-smooth subsets - and thus all constructible subsets of Zariski geometries - are all uniformily composed of irreducible components with dimension no less than the dimension of the smallest fiber.

Lemma 4.3 (Uniform Lower Bound on Fiber Dimension). Let $A$ be an irreducible pre-smooth subset of $M$, and let $S \subseteq A^{k} \times M^{\ell}$ be closed and irreducible. Let pr be a projection map with domain containing $S$ such that $\operatorname{pr}(S)=A$ and let $r=$ $\min _{\bar{a} \in \operatorname{pr}(S)} \operatorname{dim}\left(S\left(\bar{a}, M^{\ell}\right)\right)$. Then for each $\bar{a} \in A^{k}$ every $C \subseteq S\left(\bar{a}, M^{\ell}\right)$ has dimension no 
less than $r$. In particular, if $\operatorname{dim}\left(S\left(\bar{a}, M^{\ell}\right)\right)=r$, then all components of $S\left(\bar{a}, M^{\ell}\right)$ have dimension $r$.

Reference. Proved as Proposition 3.6.2 in [14] in Chapter 3, Section 6.

With this in mind, we can go ahead and distinguish between local dimension, or dimension of a fiber at a point, and the ordinary definition of dimension we have used until now. This lemma then should indicate that this local dimension of any fiber should always be bounded below by the smallest of the local dimensions they could have. Furthermore, this should also give us an expanded context for what pre-smoothness means for a Zariski structure.

Definition 4.4 (Local Dimension). Let $D$ be a definable set and let $b \in D$. We define the local dimension of $b$ in $D$ as

$$
\operatorname{dim}_{b}(D)=\max \{\operatorname{dim}(C): C \subseteq D \text { is an irreducible component and } b \in C\}
$$

With this notion of local dimension, we can actually show that pre-smoothness of constructible sets can actually be described as a local property.

Corollary 4.5 (Local Dimension definition of Pre-smoothness). Let $A$ be a definable subset of $M$. Then $A$ is pre-smooth if and only if for any $k, m \in \mathbb{Z}^{+}$and any relatively closed subsets $S_{1}, S_{2} \subseteq_{c l} A^{k} \times M^{m}$ it holds that

$$
\operatorname{dim}_{x}\left(S_{1} \cap S_{2}\right) \geq \operatorname{dim}_{x}\left(S_{1}\right)+\operatorname{dim}_{x}\left(S_{2}\right)-\operatorname{dim}\left(A^{k} \times M^{m}\right) \text { for any } x \in S_{1} \cap S_{2}
$$

There's another corollary about pre-smooth sets that will be useful for us later when we discuss local functions and talk about different formulations of the implicit function theorem in context of Zariski geometries. 
Corollary 4.6 (Open subsets of Pre-smooth components are pre-smooth). If $A$ is irreducible and pre-smooth, then any open subset $B \subseteq_{o p} A$ is also pre-smooth.

Strong pre-smoothness, in addition to being a characterizing property of Zariski geometries, is also preserved under elementary extensions. To prove this, we will need the following lemma

Lemma 4.7 (Pre-Smoothness of Constructible Sets transfers over Extensions). Let A be an irreducible, pre-smooth set definable in $M$ and let $S \subseteq A^{k} \times M^{\ell}$ is closed and irreducible. Let pr be a projection of $S$ such that $\operatorname{pr}(S)=A^{k}$ and $r=\min _{\bar{a} \in \operatorname{pr}(S)}\left\{\operatorname{dim}\left(S\left(\bar{a}, M^{\ell}\right)\right)\right\}$. Then for every $\bar{a}^{\prime} \in A^{k}(' M)$, every component of $S\left(\bar{a}^{\prime}, ' M\right)$ has dimension no less than $r$. In particular, if $\operatorname{dim}\left(S\left(\bar{a}^{\prime}, ' M\right)\right)=r$, then all components of $S\left(\bar{a}^{\prime}, ' M\right)$ have dimension $r$.

Now we prove the preservation theorem.

Proposition 4.8 (Strong Pre-Smoothness is preserved under Elemenetary Extension). Let $M$ be a topological structure with dimension satsifying (sPS). Then if ${ }^{*} M \geqslant M$, then ${ }^{*} M$ also satisfies (sPS).

Outline. We let $C$ be a definable irreducible subset of $M^{r+n}$ and let $\bar{c} \in{ }^{*} M$ and $C\left(\bar{c},{ }^{*} M^{n}\right)$ is an irreducible subset of ${ }^{*} M^{n}$. We can find $C_{0}$ open subset of $C$ and then we note $C_{0}\left(\bar{c},{ }^{*} M^{n}\right)$ is an open subset of $C\left(\bar{c},{ }^{*} M^{n}\right)$. We then take irreducible subsets $S_{1}$ and $S_{2}$ of $C_{0}$ and take the irreducible decomposition of the fibers of the intersection $S_{1} \cap S_{2}$. Then without loss of generality, we can assume that the projections of $S_{1}$, $S_{2}, C$, and all the irreducible components of the fiber over $S_{1} \cap S_{2}$ are a locus of some point over $M$. You then take an element of this locus and consider that the fiber of $S_{1} \cap S_{2}$ over that locus has fibers all of the same dimension by Proposition 3.33, and 
that the irreducible components of the fibers all have the same dimension as the total fibers themselves by Lemma 4.7, making us have that every component of the fibers over $S_{1}$ and $S_{2}$ satisfies the pre-smoothness inequality.

With all these things said about Zariski geometries, let us look at an example of Zariski geometries in context of algebraic varieties to get a better understanding about what properties they give us.

Following our motivations for Zariski geometries, one can prove that the Zariski topologies on smooth algebraic varieties over uncountable fields are also define Zariski geometries. This follows from our previous theorem that every algebraic variety is a Zariski structure. Also since subvarieties of smooth varieties are smooth, we know that all of the subvarieties of the smooth variety will be pre-smooth, including constructible subsets.

The main result we want to note about algebraic varieties will be our motivating theorem for classifying Zariski geometries. In fact, our classification theorem of Zariski geometries will essentially be a generalization of the following theorem.

Theorem 4.9 (Smooth semi-covering of algebraic curves). Let $C$ be an irreducible algebraic curve over an $K \vDash A C F$ and $\left\{a_{1}, a_{2}, \ldots, a_{n}\right\}$ is the set of all singular points of $C$. Then

(1) There is a smooth algebraic curve $A$ and a regular finite-to-one map $f: A \rightarrow C$ such that $f$ is a biregular bijection on $C \backslash\left\{a_{1}, a_{2}, \ldots, a_{n}\right\}$

(2) $C$ is pre-smooth if and only if $f$ is a bijection.

References. Part (1) of this theorem is proven from content in [9] using the resolution of singularities technique found as Chapter 2, Section 2, Theorem 2.12 to develop 
the method of normalization, found in Chapter 2, Section 5 of the same book. Part (2) of the theorem is proven in [14].

With some of these basic results established about Zariski geometries, let us start investigating more precisely what makes these Zariski structures appear more geometrical in flavor than ordinary Zariski structures.

\subsection{Coverings and Local Functions in Zariski Geometries}

We would like to start studying how the covering notion we developed in our theory of Zariski structures interacts with our strong pre-smoothness and pre-smoothness properties in more detail. Our goal will be to use the notion of coverings on Zariski geometries to define a notion of local functions, which will be useful to develop a theory of infinitesmial analysis on Zariski geometries. In particular, we will develop an implicit function theorem for Zariski geometries and turn our attention towards using these functions to study families of curves in Zariski geometries.

First we will recall coverings, and develop some of the additional properties that coverings have over pre-smooth sets. The first property will be developed in this proposition, which will be useful when generally discussing coverings of pre-smooth sets. We present it here without proof, but the proof can be found in [14].

Proposition 4.10 (Combinatorial Dimension captures Dimension of Generic Fibers). Let $F$ be an irreducible covering of a pre-smooth set $D$. Let $(a, b) \in F$ such that a is regular in $D$ for $F$. Then for each $a^{\prime} \in \mathcal{V}_{a} \cap^{*} D$, there is a $b^{\prime} \in \mathcal{V}_{b}$ such that $\left(a^{\prime}, b^{\prime}\right) \in{ }^{*} F$ and $\operatorname{cdim}\left(b^{\prime} / a^{\prime}\right)$ is equal to the dimension of a generic fiber of $F$.

Next, we develop the following lemma, which indicates to us that we can define a notion of multiplicity of coverings at points, which will provide us a means to count, for 
any infinitesmial of a base point of a fiber of a covering, the number of infinitesimals of a fiber element that map to that infinitesimal of the base point. In particular, the next lemma tells us the multiplicity will be maximized at generic points, as so generic points will serve as the best representative points for multiplicity. The proof for this lemma can be found in [14] as Lemma 3.6.6.

Lemma 4.11 (Maximized Multiplicities at generic base points). Let $F \subseteq D \times M^{k}$ be an irreducible finite cover of $D$ in $\bar{a}$ where $D$ is pre-smooth. If $F(\bar{a}, \bar{b})$ and $\bar{a}^{\prime} \in \mathcal{V}_{\bar{a}} \cap D\left({ }^{*} M\right)$ is generic in $D$, then

$$
\left|F\left(\bar{a}^{\prime},{ }^{*} M\right) \cap \mathcal{V}_{\bar{b}}\right| \geq\left|F\left(\bar{a}^{\prime \prime},{ }^{*} M\right) \cap \mathcal{V}_{\bar{b}}\right| \text { for each } \bar{a}^{\prime \prime} \in \mathcal{V}_{\bar{a}} \cap D\left({ }^{*} M\right)
$$

This lemma justifies the following definition for multiplicity.

Definition 4.12 (Multiplicity). Let $(\bar{a}, \bar{b}) \in F$ and $F$ be a finite covering of $D$. We define the multiplicity of $F$ at $\bar{a}$ over $D$ as :

$$
\operatorname{mult}_{\bar{b}}(\bar{a}, F / D)=\left|F\left(\bar{a}^{\prime},{ }^{*} M^{k}\right) \cap \mathcal{V}_{\bar{b}}\right| \text { for any } \bar{a}^{\prime} \in \mathcal{V}_{\bar{a}} \text { generic in } D \text { over } M
$$

Also, assuming $\bar{a} \in \operatorname{reg}(F / D)$ we define

$$
\operatorname{mult}(a, F / D)=\sum_{\bar{b} \in F\left(\bar{a}, M^{k}\right)} \operatorname{mult}_{\bar{b}}(\bar{a}, F / D)
$$

An fairly trivial property to note about multiplicity is the following, which is partly motivated by the lemma as well.

Proposition 4.13 (Invariance of Multiplicity under Change of Base Point). Let D be pre-smooth and let $\bar{a} \in \operatorname{reg}(F / D)$. Then 


$$
\operatorname{mult}(a, F / D)=\left|F\left(\bar{a}^{\prime},{ }^{*} M^{k}\right)\right|
$$

for $\bar{a}^{\prime} \in D\left({ }^{*} M\right)$ generic over $M$ (not necessarily in $\left.\mathcal{V}_{a}\right)$. Furthermore, this number does not depend on the choice of $\bar{a} \in D$.

With a notion of multiplicity defined, we can use this notion to define a notion of an unramified cover, which will be a cover where each infinitesimal of the base point is mapped to by precisely one infinitesimal for each fiber point.

Definition 4.14 (Unramified Covering). Let $F$ be a finite cover of $D$. We say that $F$ is unramified at $(\bar{a}, \bar{b}) \in F$ if $\operatorname{mult}_{\bar{b}}(\bar{a}, F / D)=1$. Furthermore, we can define :

$$
\operatorname{unr}(F / D)=\left\{(\bar{a}, \bar{b}) \in F: \operatorname{mult}_{\bar{b}}(\bar{a}, F / D)=1\right\}
$$

With the notion of an unramified cover, we can now start discussing situations in which a cover of a definable subset of a Zariski geometry defines an implicit function from the base space of the covering to the fibers. In particular, let us start by defining the notion of a local function.

Definition 4.15 (Local Functions and Isomorphisms). Let $F \subseteq D \times M^{k}$ be a definable relation and $(a, b) \in F$. We say $F$ defines a local function from $\mathcal{V}_{a} \cap D$ into $\mathcal{V}_{b}$ if the restriction of $F$ to $\mathcal{V}_{a} \times \mathcal{V}_{b}$ is the graph of a function from $\mathcal{V}_{a} \cap D$ to $\mathcal{V}_{b}$

Now let $F \subseteq D \times R$ be a finite-to-finite irreducible relation which is relatively closed in $F \times R$ and $\operatorname{pr}_{D}(F)=D$ where $\pi_{D}$ is the projection function onto $D$. We now say $F$ defines a local function from $D$ to $R$ if for every $(a, b) \in F$, we have $F$ defining a local function from $\mathcal{V}_{a}$ to $\mathcal{V}_{b}$ - that is, $\left.F\right|_{\mathcal{V}_{a} \cap \mathcal{V}_{b}}$ is a bijection between $\mathcal{V}_{a} \cap D$ and $\mathcal{V}_{b} \cap R$.

If $F$ is a local function from $D$ to $R$ with $\pi_{R}(F)=R$, then we call $F$ a local isomorphism from $D$ to $R$. 
Given this definition of a local function, we can see almost immediately from the definition of unramified points, the definition of local function, and from Lemma 4.3.

Corollary 4.16 (Unramified Points define Local Functions). Let $F \subseteq D \times M^{k}$ be, generically, a finite covering of $D$ and $D$ be pre-smooth. Then $F$ is unramified at a point $(a, b) \in F$ if and only if $F$ defines a local function from $\mathcal{V}_{a}$ to $\mathcal{V}_{b}$. In particular, if

$$
D_{1}=\{a \in \operatorname{reg}(F / D): \forall b(F(a, b) \rightarrow(a, b) \in \operatorname{unr}(F / D))\}
$$

then $F$ defines a local function on $D_{1}$.

Theorem 4.17 (Implicit Function Theorem). Let $M$ be a strongly pre-smooth Zariski structure, $D \subseteq M^{n}$ be irreducible, and $F \subseteq D \times M^{r}$ be an irreducible finite covering of $D$ with $\operatorname{dim}(F)=\operatorname{dim}(D)$. Then there is an open dense subset $D_{1} \subseteq D$ such that $F \cap\left(D_{1} \times M^{r}\right)$ defines a local function on $D_{1}$.

Proof. We note by strong pre-smoothness that there is a $D_{1} \subseteq D$ such that $D_{1}$ is pre-smooth, open, and dense in $D$. This means that $F \cap\left(D_{1} \times M^{r}\right)$ will form a local function on $D_{1}$ by the preceding corollary.

We can further expand the usefulness of this result with the following theorem. In particular, in the corollary we used to prove the Implicit Function Theorem, we can replace the assumption that $D$ is pre-smooth and replace it with the assumption that $M$ is a one-dimensional, pre-smooth Zariski structure. It also allows us to replace the assumption that $M$ is strongly pre-smooth with the assumption that $M$ is onedimensional and pre-smooth in the Implicit Function Theorem. We omit the proof here, but a proof of this theorem can be found as Theorem 3.6.21 in [14] in Chapter 3, Section 6. 
Theorem 4.18. Let $M$ be a one-dimensional, uncountable, pre-smooth, irreducible Zariski structure. Then $M$ is a Zariski geometry.

To prove this theorem, Zilber first proves the following lemma, which provides a useful fact about coverings of pre-smooth sets in general.

Lemma 4.19 (Induced Pre-Smoothness of Local Functions). Let $F \subseteq D \times M^{k}$ where $F$ is an irreducible cover of $D$ defining a local function on $D$. If $D$ is pre-smooth, then so is $F$.

Finally, we end our discussiong by noting that our notion of local isomorphism allows us to finally strengthen pre-smoothness by saying a subset it smooth if and only if it is locally isomorphic to a Zariski curve.

Definition 4.20 (Smooth Set). Given $M$ is a one-dimensional, pre-smooth Zariski structure, we call a definable $D \subseteq M^{n}$ smooth if $D$ is locally isomorphic to an open subset of $M^{k}$ for some $k$.

We note without proof the following theorem, which lists some fairly trivial examples of smooth sets.

Theorem 4.21 (Smoothness Theorem). Assuming $M$ is a one-dimensional presmooth Zariski structure, the following are all true

(1.) Any open subset of $M^{n}$ is smooth.

(2.) For every irreducible definable $D \subseteq M^{n}$, there is an open irreducible $D_{0} \subseteq D$ which is smooth.

(3.) If $D_{1}$ and $D_{2}$ are smooth definable relations, then so is $D_{1} \times D_{2}$ 
With the preceding theory now developed, we can take these results to begin constructing new Zariski structures and geometries from old ones in more robust ways. In particular, we will try to get a rough generalization of moduli spaces from more standard treatments of geometry in the notion of topological sorts, and we will use constructs like these to study families of curves and what it means for them to be tangent in our context.

\subsection{Well-Behaved Kinds of Zariski Geometries}

To begin our discussion of constructing new kinds of nice Zariski structures, we will define the following notion of a topological sort, which can be thought as a kind of quotient map of a substructure of a Zariski structure along some mutually disjoint closed subsets of the Zariski structure.

Definition 4.22 (Topological Sort). Let $N$ be an irreducible subset of $M^{n}$ and let $E$ be a relatively closed equivalence relation on $N$. A subset $T \subseteq(N / E)^{k}$ is called closed in $(N / E)^{k}$ if $p^{-1}(T)$ is closed in $N^{k}$. The set $(N / E)$ together with the structure of the closed subsets on them will be called a topological sort in $M$. In particular, let us define the equivalence relation $E^{(k)}$ by

$$
\left(a_{1}, \ldots, a_{k}\right) E^{(k)}\left(b_{1}, \ldots, b_{k}\right) \Leftrightarrow a_{i} E b_{i} \text { for each } i=1, \ldots, k
$$

so we may identify $(N / E)^{k}$ with $N^{k} / E^{(k)}$ by the natural mapping preserving the equivalence relation $\sigma:\left(\left[x_{1}\right], \ldots,\left[x_{k}\right]\right) \mapsto\left[\left(x_{1}, \ldots, x_{k}\right)\right]$. Then we see the quotient maps $p_{k}: N^{k} \rightarrow N^{k} / E^{(k)}$ define the quotient topology on each $N^{k} / E^{(k)}$. 
As a remark on notation, for a definable equivalence relation $E$, we may sometimes use $E(a, b)$ to say an element $a$ is equivalent to $b$, or we may use $a E b$ as we used in the definition above. Generally, we will use the formula $E(x, y)$ for the relation whenever we want to use it to reference its definable sets, such as $[a]=E(a, M)$.

To clarify an earlier remark : topological sorts correspond to moduli spaces in the sense that they identifiy closed subsets (think objects like varieties, which more or less correspond to curves and surfaces in our ordinary algebro-geometric setting) as indivisible objects represented by our equivalence classes, and we've taken a quotient of our space to identify these objects with individual points. This essentially collapses our space into one where the points represent some definable objects of our geometry, and the resulting quotient space codifies some sense of closeness this object has with the other classes we've defined along with it. To see a concrete example of this, consider the following examples

Example 4.23 (Projective Spaces as Topological Sorts). Let $\mathbb{A}^{n+1}(K)$ be the affine $n$-space over a field $K$. Consider the irreducible subspace $N=\mathbb{A}^{n+1}(K) \backslash\{\overline{0}\}$ where $\overline{0}$ is the origin of the affine space. Let us then define the equivalence relation by

$$
E=\{(\bar{x}, \bar{y}) \in N \times N: \exists \lambda \in K \text { such that } \bar{x}=\lambda \cdot \bar{y}\}
$$

where here, $\bar{x}=\lambda \cdot \bar{y}$ is understood as representing a system of equations rather than a vector arithmetic expression. Hence the equivalence relation is definable on $N$. We call $\mathbb{P}^{n}(K)=N / E$ with its quotient topological structure induced from this relationship the projective $n$-space of $K$. We note that the natural quotient of this map sends a point to its homogeneous coordinate representation: 


$$
\theta_{n}: N \rightarrow N / E \quad \text { by } \quad \theta_{n}:\left(x_{0}, x_{1}, \ldots, x_{n}\right) \mapsto\left[x_{0}, x_{1}, \ldots, x_{n}\right]
$$

The quotient topology is the Zariski topology of projective space, and the closed subsets of this space are called projective varieties.

The following fact about topological sorts is not only something useful that we hoped to get by design, but it is also fairly trivial to prove from the definitions. It will be presented without proof.

Proposition 4.24 (Topological Sorts are Noetherian Topological Structures). If N/E is a topological sort, then $N / E$ is a topological structure satisfying (DCC) and $(S P)$. Furthermore, $N / E$ is complete if $M$ is complete.

The next proposition will also contains some fairly trivial propositions to prove, and they will also be useful for us whenever we develop some of the theory of topological sorts.

Proposition 4.25 (Some facts about Topological Sorts). The following are true about topological sorts :

(1.) The map $p_{1}: N \rightarrow N / E$ is a continuous, closed, and open map.

(2.) A subset $T \subseteq(N / E)^{k}$ is irreducible if and only if there is an irreducible $S \subseteq N^{k}$ such that $\operatorname{pr}(S)=T$.

With topological sorts in mind, we might be interested in how the dimension of notion from $N$ (or rather $M$ ) transfers to the topological sort. There is some legwork needed to demonstrate that dimension can be transferred at all, but we will particularly use the following definition : 
Definition 4.26 (Dimension of Irreducible Closed Sets in Topological Sorts). Let $N / E$ be a topological sort, and assume $T=p_{k}(S) \subseteq(N / E)^{k}$ for some $S \subseteq N^{k}$ which is closed and irreducible. The dimension of $T$ in $N / E$ is defined as

$$
\operatorname{dim}(T / S)=\operatorname{dim}(S)-\min _{t \in T}\left\{\operatorname{dim}\left(p_{k}^{-1}(t) \cap S\right)\right\}
$$

The legwork in choosing the definition mostly consists in demonstrating the choice of $S$ does not affect the dimension. We will omit the demonstration that this is the case, but this fact is demonstrated in [14] as Lemma 3.7.5. So we can simply define $\operatorname{dim}(T)=\operatorname{dim}(T / S)$ for any $S$ closed and irreducible such that $p_{k}(S)=T$. We can go ahead and start talking about the properties preserved under the map. We also can extend the notion of dimension to all closed sets, recalling that our topological sorts are Noetherian.

Definition 4.27 (Dimension of Closed Sets in Topological Sorts). Let $T$ be a closed set of a topological sort $N / E$, and let $T_{1}, \ldots, T_{n}$ be the irreducible components of $T$ in $N / E$. Then

$$
\operatorname{dim}(T)=\max _{1 \leq i \leq n} \operatorname{dim}\left(T_{i}\right)
$$

Proposition 4.28 (Topological Sorts inherit some Nice Dimension Properties). Let $(N / E)$ be a topological sort with dimension notion inherited from $N$. Then $(N / E)$ satisfies (DP), (DU), and (SI).

Outline. (DP) is trivial, and (DU) follows fairly easily from (SI); but (SI) probably can use an inequality argument on the minimum subtracted from the dimension of the lifted sets when doing the lifts. 
Unfortunately there are counterexamples that show the properties (FC) and (AF) are not necessarily preserved when topological sorts are constructed from topological structures. Such a counterexample is given in [14] with a topological sort build from the topological structure on the projective line - and it fails both axioms simultaneously. On the other hand, we can further isolate some kinds of topological sorts that actually define full Zariski structures. We will call these Zariski sets, or more simply Z-sets.

Definition 4.29 (Zariski Set). A topological sort in $M$ satisfying all the axioms of a Zariski structure will be called a Zariski set, or Z-set, in $M$.

We also have a notion of morphisms between such sets defined as follows

Definition 4.30 (Zariski Morphism). Given two topological sorts $T_{1}$ and $T_{2}$ and a pair of subsets $S_{1} \subseteq T_{1}^{k}$ and $S_{2} \subseteq T_{2}^{l}$, we call a map $\varphi: S_{1} \rightarrow S_{2}$ a (Zariski) morphism, or Z-morphism if the graph of $\varphi$ is closed in $S_{1} \times S_{2}$. If $\varphi$ is a bijection, we say that $\varphi$ is an isomorphism between $S_{1}$ and $S_{2}$.

One easy fact following from the definition of Z-morphism is that they all are automatically continuous maps between the induced subspace topologies of their domains and codomains, since we can show that preimages of closed sets are closed under such maps using the axioms of topological structures. We now provide our first example of topological structures which turn out to satisfy the Zariski structure axioms.

Definition 4.31 (Pre-Manifold). A topological sort $T$ is called a pre-manifold in $M$ if there exists a finite collection $U_{1}, U_{2}, \ldots, U_{k}$ of subsets which are open and dense in $T$ such that 
(1.) $T=U_{1} \cup U_{2} \ldots \cup U_{k}$

(2.) For every $i \leq k$, there is an irreducible subset $V_{i} \subseteq M^{n}$, pre-smooth in $\mathrm{M}$, and an isomorphism $\varphi_{i}: U_{i} \rightarrow V_{i}$.

Proposition 4.32 (Pre-manifolds are nice Z-sets). Every pre-manifold is an irreducible, pre-smooth Z-set.

Proof. Note that each $U_{i}$ is irreducible by isomorphism. If $T=S_{1} \cup S_{2}$ for some closed subsets $S_{1}$ and $S_{2}$, then we know $U_{i} \subset S_{j}$ for some $j \in\{1,2\}$. But since we know $U_{i}$ is dense, we then see that $\overline{U_{i}}=T \subseteq S_{j}$. So $T=S_{j}$, and thus $T$ is irreducible. Next we note that we have already proven that topological sorts satisfy the axioms to be Noetherian topological structures whose dimension notions satisfy (DP), (DU), and (SI). Thus we only need to show the dimension notion on $T$ satisfies (AF) and (FC), and that $T$ is also pre-smooth. Since these are local properties, it is sufficient to prove the theorem on each chart $U_{i}$. So for $(\mathrm{AF})$ and $(\mathrm{FC})$, we need to prove the theorem on each $\operatorname{pr}\left(S \cap U_{i}\right)$ instead of each $\operatorname{pr}(S)$, and prove (PS) on $S_{1} \cap U_{i}$ and $S_{2} \cap U_{i}$ instead of $S_{1}$ and $S_{2}$. It is trivial to verify these conditions hold, since isomorphisms between topological sorts will preserve open and closed sets in both directions, as well as dimension.

The notion of a pre-manifold can be strengthened to a notion of a manifold, which will be the kind of structure that we allow to constitute a family of curves in the next chapter.

Definition 4.33 ( $n$-Manifold). Let $M$ be a Zariski structure and $C$ be an irreducible pre-smooth Zariski structure of dimension 1 (sometimes $C$ is called a Zariski curve). Let $U$ be a Z-set in $M$. We call $U$ an $n$-manifold with respect to $C$ if $U$ is locally isomorphic to $C^{n}$, for some $n$. 
With some of the basic building blocks of Zariski structres expanded to the context of Zariski geometries, and with the notions we have defined notions of a more explicitly geometric nature for Zariski geometries, we can now start putting these pieces together to construct the objects used to classify Zariski geometries. In particular, we want to start talking about the families of curves definable in Zariski geometries, and understand how tangency between curves is defined inside these structures.

\subsection{Curves, Branches, and Tangency}

Throughout the development of this chapter, we will assume that $C$ refers to a one-dimensional, irreducible, essentially uncountable Zariski structure on the universe $C$. In other words, we will assume that $C$ is a nice Zariski curve. We will also assume that our saturated elementary extension ${ }^{*} C$ of $C$ is a universal domain in the model-theoretic sense, which will guarantee that every definable set in ${ }^{*} C$ will contain generic points. In this kind of Zariski curve, we can develop a rich geometry which readily admits a well-behaved definition for families of curves and some notion of tangency between curves. This notion of geometry still admits singularities, however; so the theory of tangency will need to refer to tangency between different branches of curves instead to account for different tangential trajectories that could occur near a singular point. These tools will eventually allow us to start defining a nice algebraic structure on branches of curves up to tangency, and give us a nice way to algebratize these kinds of Zariski geometries.

Definition 4.34 (Definable Family of Curves). A (definable) family of curves in $C^{m}$ is defined as a triple $(P, L, I)$ where $P$ is an open subset of $C^{m}, L$ is a $k$-manifold 
with respect to $C^{k}$ for some $k \geq 1, I \subseteq P \times L$ is a irreducible relation in $P \times L$, closed in $P \times L$, and :

(1.) The projections of $I$ cover $P$ and $L$ respectively.

(2.) For every $l \in L$, the set $I(P, l)$ is one-dimensional, and for $l$ generic the set is irreducible.

We call $I$ an incidence relation on $L$. We also say our family of curves is faithful if additionally :

(3.) For any $l_{1}, l_{2} \in L$, the intersection $I\left(P, l_{1}\right) \cap I\left(P, l_{2}\right)$ is at most finite, provided $\operatorname{acl}\left(l_{1}\right) \neq \operatorname{acl}\left(l_{2}\right)$

We also say that the family of curves passes through $p \in C^{m}$ if for every $l \in L$, the relation $I(p, l)$ holds.

Generally, when it's clear from context, we say that $L$ represents the family of curves, and we identify every curve $l \in L$ with $I(P, l)=\left\{p \in C^{m}: I(p, l)\right\}$.

One detail to note about these families of curves is that we can remove small closed subsets of points so that the only points which remain in our family are ones incident on at least one curve, and so no points belong to almost all curves. In other words $\operatorname{dim}(p, L)<\operatorname{dim}(L)$ for every $p \in P$.

An axiom that we want for our definable curves which will be important for proving our classification theorem for Zariski geometries will be an assumption that the space has an abundance of curves.

Definition 4.35 (Ample Zariski Geometry). $C$ is called ample if it satisfies the condition :

-(AMP) Ampleness : There is a two-dimensional, irreducible, faithful family $L$ of curves on $C^{2}$ which is locally isomorphic to an open subset of $C^{2}$. 
In particular, one fact that follows from this assumption of ampleness is that if we take the family of curves $L$ on $C^{2}$ guaranteed by the property and a generic point $(a, b) \in C^{2}$, then we can define

$$
L_{(a, b)}=I((a, b), L)
$$

which represents a family of curves on $C^{2}$ through $(a, b)$ with incidence relation $I \cap$ $\left(L_{(a, b)} \times C^{2}\right)$. It can be shown that this family of curves as $\operatorname{dim}\left(L_{(a, b)}\right)=1$ and by the Smoothness Theorem in the previous chapter, we can choose a one-dimensional, irreducible, smooth subfamily of curves $G \subseteq L_{(a, b)}$ which passes through $(a, b)$. In other words, we have the following proposition :

Proposition 4.36 (Ample Geometries have an Ample Supply of Curves). Let $C$ be ample. For any $(a, b) \in C^{2}$ there is an irreducible, faithful, one-dimensional, smooth family $G$ of curves through $(a, b)$.

With some notion of families of curves defined, we now want to define an abstract notion of trajectories for curves. In particular, we want to create an object that mimicks something like building a curve with all of its tangent vectors by introducing something like a line bundle

Definition 4.37 (Branch of a Curve at a Point). Let $(a, b)$ be a point in $C^{2}$. A subset $\gamma \subseteq \mathcal{V}_{(a, b)}$ is said to be a branch at a curve at $(a, b)$ if there are $m \geq 2, \bar{c} \in C^{m-2}$, and irreducible, smooth family $G$ of curves through the concatenated tuple $(a, b)^{\wedge} \bar{c}$ with an indicence relation $I$ and a curve $g \in G$ such that the cover $I$ of $G \times C$ defined by

$$
\left(u,(x, y)^{\frown} \bar{z}\right) \mapsto(u, x)
$$


is regular and unramified at $\left(g,(a, b)^{\wedge} \bar{c}\right)$, and for some $g^{\prime} \in \mathcal{V}_{g} \cap G\left({ }^{*} C\right)$

$$
\gamma=\left\{(x, y) \in \mathcal{V}_{(a, b)}: \exists \bar{z} \in \mathcal{V}_{\bar{c}} \text { such that }\left(g^{\prime},(x, y)^{\frown} \bar{z}\right) \in I\right\}
$$

One characterization we want to make about branches of curves at points is to realize that they can essentially be characterized by local functions passing through $(a, b)$ with their trajectories characterized by the corresponding points $\bar{c}$ for each branch. In particular, we will call $\gamma=\tilde{g}^{\prime}$ from this definition, which is graph of a local function from $\mathcal{V}_{a}$ to $\mathcal{V}_{b}$ by Proposition 4.10 regarding combinatorial dimension. We call this function the local function from $\mathcal{V}_{a}$ to $\mathcal{V}_{b}$ in family $G$ with trajectory $\bar{c}$.

Proposition 4.38 (Faithful Representation of Branches by Local Functions). Let $G$ be an irreducible, faithful, smooth family of curves through $(a, b)$ and $\left(g_{1}, g_{2}\right) \in{ }^{*} G=$ $G\left({ }^{*} M\right)$. If $\tilde{g}_{1}=\tilde{g}_{2}$ as functions from $\mathcal{V}_{a} \rightarrow \mathcal{V}_{b}$, then $g_{1}=g_{2}$.

Proof. This is essentially trivial from the faithfulness axiom for families of curves, which is (3) under the definition of a family of definable curves.

Definition 4.39 (Branch Tangency Relation). Let $I_{1}$ and $I_{2}$ be two families of local functions from $a$ and $b$, with trajectories $\bar{c}_{1}$ and $\bar{c}_{2}$. We say that the corresponding branches defined by $g_{1} \in G_{1}$ and $g_{2} \in G_{2}$ are (mutually) tangent at $(a, b)$, written as

$$
g_{1} T g_{2}
$$

if there is an irreducible component $S=S_{\left(I_{1}, I_{2}, a, b, \bar{c}_{1}, \bar{c}_{2}\right)}$ of the set

$$
\left\{\left(u_{1}, u_{2}, x, y, \bar{z}_{1}, \bar{z}_{2}\right) \in G_{1} \times G_{2} \times C^{2} \times C^{m_{1}-2} \times C^{m_{2}-2}:\left(u_{1}, x, y, \bar{z}_{1}\right) \in I_{2} \text { and }\left(u_{2}, x, y, \bar{z}_{2}\right) \in I_{2}\right\}
$$


such that the following hold :

(1.) $\left(g_{1}, g_{2}, a, b, \bar{c}_{1}, \bar{c}_{2}\right) \in S$

(2.) The image of the projection

$$
\left(u_{1}, u_{2}, x, y, \bar{z}_{1}, \bar{z}_{2}\right) \mapsto\left(u_{1}, u_{2}\right)
$$

is dense in $G_{1} \times G_{2}$.

(3.) For $i \in\{1,2\}$, the images of the maps

$$
\left(u_{1}, u_{2}, x, y, \bar{z}_{1}, \bar{z}_{2}\right) \mapsto\left(x, y, \bar{z}_{i}, u_{i}\right)
$$

are dense in $I_{i}$ and the corresponding coverings are regular at the points in $\left(a, b, \bar{c}_{i}, g_{i}\right)$

One way to understand this definition is that we say two branches of curves are tangent if there is an irreducible cover that covers some dense subsets of the pairs of curves and some dense subsets of the incidence relations such that the relation defined by the cover also says that the two branches, the point at which they intersect, and their trajectories relate to each other.

Of all the facts to note about the tangency relation, one of the most powerful facts is the following equivalent formulation of tangency in terms of the requirement that at the infinitesimal neighborhood of the intersection, and every local function of that point in one of the family of curves, there is another local function in the other family of curves which mimicks its behavior at that point. In other words, tangent functions are exactly ones which locally approximate each other. 
Proposition 4.40 (Local Function Characterization of Tangency). Let $G_{1}$ and $G_{2}$ be families of curves defining local functions from a to $b$, and let $g_{1} \in G_{1}$ and $g_{2} \in G_{2}$ - both generic in their respective families. The following conditions are equivalent:

(1.) $g_{1} T g_{2}$

(2.) $\forall x \in \mathcal{V}_{a} \forall g_{1}^{\prime} \in \mathcal{V}_{g_{1}} \exists g_{2}^{\prime} \in \mathcal{V}_{g_{2}}: \tilde{g}_{1}^{\prime}(x)=\tilde{g}_{2}^{\prime}(x)$

(3.) $\forall x \in \mathcal{V}_{a} \forall g_{2}^{\prime} \in \mathcal{V}_{g_{2}} \exists g_{1}^{\prime} \in \mathcal{V}_{g_{1}}: \tilde{g}_{1}^{\prime}(x)=\tilde{g}_{2}^{\prime}(x)$

Furthermore, there are Zariski-open subsets $G_{1}^{0} \subseteq G_{1}$ and $G_{2}^{0} \subseteq G_{2}$ such that the conditions are still equivalent for any $g_{1} \in G_{1}^{0}$ and $g_{2} \in G_{2}^{0}$

Reference. Proven as Proposition 3.8.14 in [14] in Chapter 3, Section 8.

This theorem is important, not only because it gives us a convenient equivalent definition of the tangency which makes some later proofs easier, but because this equivalent definition vastly simplifies the process of proving that our notion of tangency is reflexive and transitive. The proof that tangency is indeed an equivalence relation can be found in Section 3.8 of [14]. One of the intermediate propositions which is proven in that book to establish tangency as an equivalence relation is the following, which can be used to demonstrate the symmetry of the tangency relation.

Proposition 4.41 (Dimension of Tangency classes). Let $G_{1}, G_{2}$ be smooth, faithful families of branches of curves through $(a, b)$, and let $g_{1}$ be generic in $G_{1}$. Then $T\left(g_{1}, y\right)$ of curves in $G_{2}$ tangent to $g_{1}$ is at most of dimension $\operatorname{dim}\left(G_{2}\right)-1$.

Furthermore, if the tangency relation $T \subseteq G_{1} \times G_{2}$ densely projects on $G_{1}$ or $G_{2}$, then $\operatorname{dim}(T)=\operatorname{dim}\left(G_{1}\right)+\operatorname{dim}\left(G_{2}\right)-1$. In particular, this will be the case when $G_{1}=G_{2}$. 
Using this proposition, we motivate the following definition, and use it to establish precisely how tangency defines an equivalence relation.

Definition 4.42 (Sorts of Branches of Curves Through a Point). Let $(a, b) \in C^{2}$, and let $\bar{c} \in C^{m}$ for some $m \in \mathbb{N}$ be a trajectory of a the family of the definable, irreducible, smooth family of curves $G_{\bar{c}, i}$ through $(a, b)$ where $i$ denotes an arbitrary indexing scheme for the families of curves.

$$
\mathcal{G}_{(a, b)}=\bigcup G_{\bar{c}, i}
$$

The tangency relationship is specifically defined over $\mathcal{G}_{(a, b)}$, and as we have stated and will use in the following chapter

Lemma 4.43 (Tangency Equivalence Relation). $T$ defines an equivalence relation $\operatorname{over} \mathcal{G}_{(a, b)}$.

Now let us work towards using tangency to classify Zariski geometries.

\subsection{Obtaining the Z-field and Classification Theorem}

We will now aim to develop a group structure from the tangency classes of a Zariski curve, use this group structure to develop a field from the curve, and then use this to prove a fundamental classification result of Zariski geometries.

We start by explicitly stating the goal we have in mind for defining a group structure on our curve. We want to find some Zariski structrure on a manifold, such as a Zariski curve, which defines a group operation. More precisely, we want the following 
Definition 4.44 (Z-group). Let $G$ be a manifold and $P \subseteq G^{3}$ a closed ternary relation which is the graph of a binary operation

$$
(u, v) \mapsto u \cdot v
$$

over $G$. We say that $(G, P)$ is a $Z$-group if $(G, \cdot)$ is a group for the binary operation defined by $P$.

To work towards building such a group, let us start by trying to build a group structure out of the tangency classes from the previous chapter. First we note that our branches at a point $(a, b)$ will define local functions from $\mathcal{V}_{a}$ to $\mathcal{V}_{b}$. Let us try to instead find a way to work with local functions from $\mathcal{V}_{a}$ to itself at each point $(a, b)$ by using some of the following constructs.

Definition 4.45 (Inverse Family of Curves). Let $g \in G_{c} \subseteq \mathcal{G}_{(a, b)}$ and identify $g$ with its local function $\tilde{g}: \mathcal{V}_{a} \rightarrow \mathcal{V}_{b}$. We define the inverse as

$$
g^{-1}=\left\{(y, x) \in \mathcal{V}_{b} \times \mathcal{V}_{a}:(x, y) \in g\right\}
$$

and define the inverse family $G_{c}^{-1}$ to be a copy of the definable set $G_{c}$ but with the inverse action induced by its elements mapping $\mathcal{V}_{b} \rightarrow \mathcal{V}_{a}$.

From this definition, it is fairly easy to verify the following lemma using the local function characterization of tangency in Proposition 4.40.

Lemma 4.46 (Inversion Preserves Tangency). For every $g_{1}, g_{2} \in \mathcal{G}_{(a, b)}$,

$$
g_{1} T g_{2} \quad \text { if and only if } \quad g_{1}^{-1} T g_{2}^{-1}
$$


With this inversion operation in place, we can go ahead and define the notion of composing pairs of branches of curves using the following operation.

Definition 4.47 (Composition of Curves). For any $g_{1} \in G_{c_{1}}$ and $g_{2} \in G_{c_{2}}$ with $G_{c_{1}}, G_{c_{2}} \subseteq \mathcal{G}_{(a, b)}$, define the composition curve

$$
g_{2}^{-1} \circ g_{2}=\left\{\left(x_{1}, x_{2}\right) \in C^{2}: \exists y, z_{1}, z_{2} \text { such that }\left(x_{1}, y, z_{1}\right) \in g_{1} \text { and }\left(z_{2}, y, x_{2}\right) \in g_{2}^{-1}\right\}
$$

and its branch at $(a, a)$ is defined by

$$
\left.\left(g_{2}^{-1} \circ g_{1}\right)\right)_{(a, a)}=\left\{\left(x_{1}, x_{2}\right) \in \mathcal{V}_{a} \times \mathcal{V}_{a}: x_{2}=\tilde{g}_{2}^{-1}\left(g_{1}\left(x_{1}\right)\right)\right\}
$$

Some other details about this operation are discussed in [14], but the key idea will be that this composition will define an operation which can be used to define a group structure out of some branches of curves up to tangency.

To construct our Z-group, we will first show that this composition defines a pregroup structure, which will be a partially-defined group operation on a manifold that will obey many group properties on some open set, but will more importantly be a structure which we can extend to a full group in a later result.

Proposition 4.48 (Pre-group of Jets existence). There is a one-dimensional irreducible manifold $U$ with respect to the Zariski curve $C$ and a constructible irreducible ternary relation $P \subseteq U^{3}$ which is the graph of a partial operation $\odot: U^{2} \rightarrow U$ and determines a partial Z-group structure on $U$ - that is, there is an open subset $V \subseteq U^{2}$ such that:

(1.) For any $(u, v) \in U^{2}$, there is a unique $w=u \odot v \in U$ such that $(u, v, w) \in P$

(2.) For any generic $(u, v, w) \in U^{3}$, we have $u \odot(v \odot w)=(u \odot v) \odot w$ 
(3.) For each $(u, v) \in V$, the equations

$u \odot x=v$ and $y \odot u=v$

both have solutions in $U$.

Outline. Let $N$ be the family of curves through a point $(a, b)$ obtained from the ampleness of $C$. We let $\left(N^{-1} \circ N\right)$ be a family of curves $H_{(a, a)}$ passing through the point $(a, a)$. By the dimension of tangency classes in Proposition 4.41, this tangency relation $T$ will have dimension 1 on an open subset $H$ of $N^{2}$, making $\operatorname{dim}(H / T)=1$. Since $N$ is locally isomorphic to our ample $C$, there must be an irreducible curve $S$ which interesects infintely many equivalence classes of $T$. $U=S / T$ will be our manifold, and consequently by irreducibility and dimension arguments

$$
U=S / T=H / T
$$

Finally, it can be shown (such as in Lemma 4.1.8 in [14] in Chapter 4, Section 1) that composition of branches will preserve tangency on this manifold, and so it forms a partial map, and it can be show that the composition of branches corresponds to composition of local functions, which will induce the partial map with the desired properties.

Finally, we build a Z-group from our pre-group using the following theorem. The proof of the following theorem will be summarized, but a more detail proof can be found in [14]. This theorem will establish the existence of our Z-group of jets.

Theorem 4.49 (Z-version of Weil's theorem on pre-groups). For any partial irreducible Z-group $U$, there is a connected Z-group $G$ and a Z-isomorphism between 
some dense open $U^{\prime} \subseteq U$ and a dense open $G^{\prime} \subseteq G$.

Outline. We start by building a semi-group $G$ out of the partial functions generated by translations of $U$ by elements of $U$ under the pre-group operation. In other words, the elements are of the form

$$
s_{a}: u \rightarrow a \odot u
$$

for each $a, u \in U$. We consider two elements of $G$ equal if they agree on an open subset of $U$. We can then prove that the semi-group is identifiable with a constructible sort $(U \times U) / E$ where $E$ is an equivalence relation which considers two pairs $\left(a_{1}, a_{2}\right)$ and $\left(b_{1}, b_{2}\right)$ equal if and only if they have

$$
a_{1} \odot\left(a_{2} \odot u\right)=b_{1} \odot\left(b_{2} \odot u\right) \text { for all } u \text { in an open subset of } V \subseteq U
$$

The last condition can be made constructible by restating it in terms of the dimension of the set on which the operations agree. This gives us a way of embedding the pre-group of $U$ into the semi-group $G$. Then one can prove $G$ is a group using some facts about the non-definability of the identity in the stable semi-group structures ie, a semi-group whose definable subsets cannot encode an infinite linear ordering on any $G^{n}$ for $n \in \mathbb{Z}^{+}$. (This term stable is explained in [1] in Chapter 9, Section 4). The final properties are verified using dimension arguments.

Note that by this theorem, we can take any irreducible pregroup $U$, such as the one obtained by our pre-group of jets existence theorem, and topologically embed its structure into that of the topological structure of a Z-group. In particular, we use our pre-group of jets to now build our group of jets. 
Corollary 4.50 (Group of Jets). The group of jets $J$ of a Zariski curve $C$ generated by pre-group $U=H_{(a, a)} / T$ is a connected Z-group of dimension 1 .

One fact that we can note from the ampleness of our Zariski geometry is that if we have any Zariski curve $J$ with its Abelian group structure endowed on it, then any point $(a, b)$ has a one-dimensional, smooth, faithful family of curves passing through it, and the product group structure endowed on $J^{2}$ can be used to translate this point to the origin point $(0,0)$ of the product group. For the rest of the construction of the Z-field, we will assume without loss of generality that we are working over a family of curves passing through $(0,0)$.

Let us denote the group operation on $J$ using $\oplus$, and let us represent its subtraction operation (ie, adding the inverse of the right-hand operand) by $\ominus$. The graphs of these binary operations can be shown to be Zariski isomorphic to $J \times J$ by the projection map, and the graph of $\oplus$ will be irreducible, making it an irreducible cover of $J \times J$ in particular. This will endow $\mathcal{V}_{0}$ with a commutative group structure.

Definition 4.51 (Sums and Differences of Branches). Let $\tilde{g}_{1}, \tilde{g}_{2}$ be two branches of curves in $J^{2}$. We define the sum of $\tilde{g}_{1}$ and $\tilde{g}_{2}$ as

$$
\tilde{g}_{1} \oplus \tilde{g}_{2}=\left\{(x, y): \exists z_{1}, z_{2} \in J \text { such that }\left(x, z_{1}\right) \in \tilde{g}_{1},\left(x, z_{2}\right) \in \tilde{g}_{2} \text {, and }\left(z_{1}, z_{2}, y\right) \in \oplus\right\}
$$

or in other words, in the notation of local functions from $\mathcal{V}_{0}$ to $\mathcal{V}_{0}$ :

$$
\left(\tilde{g}_{1} \oplus \tilde{g}_{2}\right)(x)=\tilde{g}_{1}(x) \oplus \tilde{g}_{2}(x)
$$

We also define an analogous relationship for defining $\ominus$ for branches of curves in $J^{2}$ 
Once again, in [14], care is taken to prove that these operations are indeed well-defined. We will omit the proofs were and instead move on to defining our multiplication for our field. To define our multiplication operation for our field, we simply take our function composition operation $\circ$ and inversion $\cdot^{-1}$ for branches of curves through $(0,0)$, which we used to get our Z-group, and observe the following property.

Lemma 4.52 (Composition distributes over Sums of Branches). Let $G_{1}, G_{2}$, and $G_{3}$ be families of curves through $(0,0)$ and let $g_{i} \in G_{i}$ for $i \in\{1,2,3\}$. Then

$$
\left(g_{1} \oplus g_{2}\right) \circ g_{3} T\left(g_{1} \circ g_{3}\right) \oplus\left(g_{2} \circ g_{3}\right) \quad \text { and } \quad g_{3} \circ\left(g_{1} \oplus g_{2}\right) T\left(g_{3} \circ g_{1}\right) \oplus\left(g_{3} \circ g_{2}\right)
$$

Proof. Let us prove left distributivity without loss of understanding the proof strategy. By definition of $\oplus$, we have for each $x \in \mathcal{V}_{0}$ and $g_{i}^{\prime} \in G_{i}$ that

$$
\begin{aligned}
\left(g_{1}^{\prime} \oplus g_{2}^{\prime}\right) \circ g_{3}^{\prime}(x) & =g_{1}^{\prime}\left(g_{3}^{\prime}(x)\right) \oplus g_{2}^{\prime}\left(g_{3}^{\prime}(x)\right) \\
& =\left(g_{1}^{\prime} \circ g_{3}^{\prime}\right)(x) \oplus\left(g_{2}^{\prime} \circ g_{3}^{\prime}\right)(x)
\end{aligned}
$$

Then we can choose, for any $z_{1}, z_{2} \in \mathcal{V}_{0}$, an element $g_{i}^{\prime} \in \mathcal{V}_{g_{i}} \cap G_{i}$ for each $i \in\{1,2,3\}$ such that $g_{i}^{\prime}\left(z_{1}\right)=z_{2}$. We then use this for any $x, y \in \mathcal{V}_{0}$ to find such $g_{i}^{\prime}$ such that $\left(g_{1}^{\prime} \oplus g_{2}^{\prime}\right) \circ g_{3}^{\prime}(x)=y$. This gives the equation above, and therefore the tangency.

We now apply our same argument we used for the existence of pre-groups to obtain the following theorem which establishes the existence of a pre-field.

Theorem 4.53 (Pre-field Existence Theorem). There is a one-dimensional manifold $U$ and constructible, irreducible ternary relations $P, S \subseteq U^{3}$ which are graphs of partial maps $U^{2} \rightarrow U$ and determine a partial Z-field structure on $U$. That is 
(1.) $P$ is a pre-group structure on $U$ with binary operation $(u, v) \mapsto u \cdot v$

(2.) $S$ is a pre-group structure on $U$ with binary operation $(u, v) \mapsto u+v$

(3.) The following distribution laws hold for the binary operations

$$
(u+v) \cdot w=u w+v w \quad \text { and } \quad w \cdot(u+v)=w u+w v
$$

The proof strategy for the Z-field existence can then be replicated from the proof of Z-group existence, but the proof strategy can be fairly unwieldy having to reconcible two binary operations simultaneously, so Zilber proves Z-field existence using an additional lemma to simplify the process in [14]. We will omit the technical details involved with using that proo and simply end on the statement of Z-field existence.

Theorem 4.54 (Z-field Existence Theorem). There exists a Z-field in the Zariski geometry $C$.

Before we prove the Zariski classification theorems, we need to develop some pieces of the projective geometry and the induced intersection theory that Zariski structures give their Z-fields. Particularly, we need to understand the Zariski structures defined on projective spaces, and how some of their properties will help us classify Zariski geometries.

Construction 4.55 (Zariski Structure on Projective Space). Let $\mathbb{P}^{n}(K)$ be projective $n$-space over the field $K$. Furthermore let $\theta_{n}: \mathbb{A}^{n+1}(K) \backslash \overline{0} \rightarrow \mathbb{P}^{n}(K)$ be the natural quotient map from $\mathbb{A}^{n+1}(K)$ to projective $n$-space. We can present $\mathbb{P}^{n}(K)$ as a Z-set by letting

$$
U_{i}=\left\{\left(x_{0}, x_{1}, \ldots, x_{n}\right) \in \mathbb{A}^{n+1}(K): x_{i} \neq 0\right\}
$$


and identifying the image $\theta_{n}\left(U_{i}\right)$ with

$$
\tilde{U}_{i}=\left\{\left(y_{0}, y_{1}, \ldots, y_{n}\right) \in \mathbb{A}^{n+1}(K): y_{i}=1\right\}
$$

via the normalization technique for homogeneous coordinates :

$$
\left[x_{0}, x_{1}, \ldots, x_{i}, \ldots, x_{n}\right] \sim\left[\frac{x_{0}}{x_{i}}, \frac{x_{1}}{x_{i}}, \ldots, 1, \ldots, \frac{x_{n}}{x_{i}}\right]
$$

Given this, we can see that $\mathbb{P}^{n}(K)=\bigcup_{i=0}^{n} \tilde{U}_{i}$ where this union satisfies the conditions for making $\mathbb{P}^{n}(K)$ a pre-manifold over $K$. By Proposition 4.32 , we then see $\mathbb{P}^{n}(K)$ is an irreducible, pre-smooth Zariski structure with $\theta_{n}$ a Z-morphism.

We cannot necessarily prove that the Zariski structure defined on $P^{n}(K)$ will be complete for our Z-field $K$, but we can weaken the condition to define a new property that it satisfies. It will turn out that this property is sufficient for the purposes we have for classifying Zariski geometries.

Definition 4.56 (Weak Completeness). A Zariski structure $N$ is called weakly complete if for any pre-smooth $P$, closed subset $S \subseteq_{c l} P \times N$, and projection pr: $P \times N \rightarrow P$ such that $\operatorname{pr}(S)$ is dense in $P$; we have that $\operatorname{pr}(S)=P$.

Proposition 4.57 (Projective Spaces are Weakly Complete). $P^{n}(K)$ is weakly complete for any field $K$.

With all the theorems so far, we can finally prove the main classification theorems in the theory of Zariski structures. Our first version of the theorem will be a generalization of the theorem which tells us that any irreducible algebraic curve has a surjective regular map onto some smooth algebraic curve which is biregular and bijective on the nonsigular points. 
Theorem 4.58 (First Classification Theorem for Zariski Geometries). Let $M$ be $a$ Zariski structure satsifying the essential uncountability axiom, and let $C$ be a nonlinear, pre-smooth Zariski curve in $M$. Then there is a non-constant continuous map

$$
f: C \rightarrow \mathbb{P}^{1}(K)
$$

(where $K$ is the Z-field interpretable in $C$ by previous results). Furthermore, $f^{-1}(x)$ is finite for each $x \in \mathbb{P}^{1}(K)$ and the images of definable relations over $C^{n}$ are constructible (in the sense of algebraic geometry) over $\left(\mathbb{P}^{1}(K)\right)^{n}$.

Proof. Uses $Z$-field $K$ constructed from Theorem 4.52. $K$ will be a 1-manifold with respect to $C$. This means there will be a finite-to-finite closed relation $F \subseteq C \times K$ projecting onto an cofinite, open subset of $D \subseteq C$ and an open subset of $R \subseteq K$. We then prove the following claim

Claim 4.59. There is a cofinite subset $D^{\prime} \subseteq C$ and a non-constant continuous map $s: D^{\prime} \rightarrow K$.

To prove this claim, pick a generic $x \in D$. Then $F(x, K)$ is finite with $n$ distinct elements $\left\{y_{1}, y_{2}, \ldots, y_{n}\right\}$. Consider the symmetric polynomials

$$
s_{i}(\bar{t})=\sum_{1 \leq k_{1}<k_{2}<\ldots<k_{i} \leq n} t_{k_{1}} t_{k_{2}} \ldots t_{k_{i}}
$$

Let $s_{i}(x)$ denote the evaluation $s_{i}\left(y_{1}, y_{2}, \ldots, y_{i}\right)$ for each point $x \in D$. At each fiber, the fiber $\left\{y_{1}, \ldots, y_{n}\right\}$ is the set of solutions of

$$
p_{x}(v)=v^{n}-s_{1}(x) v^{n-1}+s_{2}(x) v^{n-2}-\ldots+(-1)^{n} s_{n}(x)
$$


Once we've constructed these function, we can take at least one $s_{i}$ as a non-constant function with cofinite image in $K$ since $\left\{y \in K: \exists x \in D^{\prime} F(x, y)\right\}$ is cofinite in $K$. The graph of $s_{i}$ can be made constructible by possibly removing finitely-many points, which means we can get the graph of $s_{i}$ to be closed in $D^{\prime} \times K$. Therefore $s_{i}$ us continuous on $D^{\prime}$. This proves the claim.

Once the function $s: D^{\prime} \rightarrow K$ has been constructed from this claim, we take the closure of its graph in $C \times \mathbb{P}^{1}(K)$ to be the set $S . S$ is irreducible because the graph of $s$ is irreducible. By the weak completeness of projective spaces, we know $S$ is a covering of $C$. We can show $S$ is a finite cover of multiplicity 1 at generic points, which entails from Corollary 3.36 that $S$ is finite over all points in $C$. Furthermore, by Proposition 4.13, we know $S$ has multiplicity 1 at every point in $C$ as well. This means $S$ is the graph of a function $f: C \rightarrow P^{1}(K)$. This proves the theorem.

This first classification theorem gives us our most powerful tool in the theory of Zariski geometries - it tells us that under the given preconditions, we can guarantee that our Zariski geometries can be endowed with some kinds of field-theoretic algebraic structure; which means that we can study these excruciatingly abstract mathematical objects through the lens of some classical constructs of algebraic geometry, up to some singularities where the curve is many-to-one. Another classification theorem can be found in Chapter 4, Section 4 of [14], but we will not discuss Zariski geometry classification here, since this theorem is sufficient to establish what we aimed to accomplish with these previous two chapters about topological structures and Zariski geometries. We understand now that Zariski geometries can be thought of as abstract mathematical structures (in the sense of model theory) whose induced 
topological and geometrical properties from the structure makes them reducible to commutative geometry over algebraically closed fields. We would now like to apply these to understand what kinds of noncommutative structures can arise out of general Zariski structures, particularly if we build them from these classical Zariski structures which are reducible to varieties. 


\section{CHAPTER 5}

\section{NONCLASSICAL ZARISKI GEOMETRIES}

\subsection{An Example of Nonclassical Structures}

With the classification theorem in mind, we may be interested in deciding whether

our irreducible, pre-smooth Zariski structures are always interpretable in ACF from the covering map constructed. Some counterexamples to this assertion can be built by developing techniques which allow us to add some obstructive structure to our classical Zariski structures to turn them into so-called nonclassical Zariski structures. On one hand, this may seem unfortunate since this indicates that classical algebraic geometry does not universally algebratize all of our Zariski structures. On the other hand, this tells us that our new class of Zariski structures may be more expressive than what we can express in algebraic geometry, and the shared Zariski structure properties could give us a way to translate results from algebraic geometry to our new Zariski structures.

Later in the chapter, we will give some demonstrations of the new kinds of Zariski structures we encounter in the nonclassical setting, and some of these new Zariski structures will be appied in the next chapter when we finally apply our results about Zariski structures to quantum mechanics. For now, let us begin by demonstrating the basic principles of constructing some nonclassical Zariski structures from some of our ordinary examples. 
Theorem 5.1 (Non-Classical Zariski Structure Existence Theorem). There is an irreducible pre-smooth Zariski structure which is not interpretable in ACF.

We will construct this Zariski structure by beginning with an arbitrary irreducible, pre-smooth Zariski structure $(M, \mathcal{C})$, a subgroup of its Zariski-automorphism group $G \leq \operatorname{Aut}_{Z a r}(M)$, and some $\tilde{G}$ with finite subgroup $H$ such that $G$ acts freely on $\mathrm{M}$ and such that they form a short exact sequence

$$
1 \rightarrow H \rightarrow \tilde{G} \stackrel{\mathrm{pr}}{\rightarrow} G \rightarrow 1
$$

Furthermore, consider a set $X \subseteq M$ where $X$ is a representative of for each orbit of $G$ on $M$. Because $G$ is free and $X$ contains precisely one element for each $G$-orbit, we know that for each $a \in M$ the set $(G \cdot a) \cap X$ is a singleton. We will now start building an extension of $M$ using $\tilde{G}$ and $X$. Let

$$
\tilde{M}=\tilde{G} \times X
$$

and define the projection map

$$
\tilde{\operatorname{pr}}:(g, x) \mapsto \operatorname{pr}(g) \cdot x
$$

We can then interpret each group element of $\tilde{G}$ as a function on $\tilde{M}$ by

$$
f:(g, x) \mapsto(f g, x)
$$

We can then define the model-theoretic structure 


$$
\tilde{\mathcal{M}}=\left(\tilde{M},\{f\}_{f \in \tilde{G}} \cup \tilde{p r^{-1}}(\mathcal{C})\right)
$$

where the signature is defined by taking the preimages of each closed subset of $M$ under the projection map pr interpreted as precisely the subsets they represent in $\tilde{M}$, and each $f \in \tilde{G}$ is interpreted using the specification above. We then take the closed sets defining the topological structure on $\tilde{M}^{n}$ for each $n$ to be all of the positive atomic formulas of $\tilde{\mathcal{M}}$ using parameters from $\tilde{M}$.

A further point to note about the topological structure of $\tilde{M}$ is that the map $\tilde{\text { pr }}$ and the structure $M$ are both definable. $M$ is definable since it was a closed subset

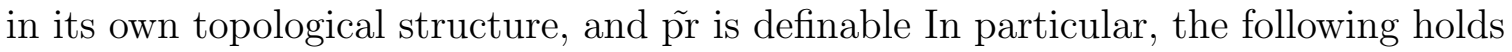
for each $f \in \tilde{G}$ :

$$
\forall x \tilde{\operatorname{pr}}(f(x))=f(\tilde{\operatorname{pr}}(x))
$$

So the image of a closed subset of $\tilde{M}^{n}$ is closed in $M^{n}$, which means we can canonically define dimension in $\tilde{M}$ as

$$
\operatorname{dim}(S)=\operatorname{dim}(\tilde{\operatorname{pr}}(S))
$$

Lemma 5.2 (Principal Extension of Zariski Structures). The isomorphism type of $\tilde{M}$ is determined by $M$ and $\tilde{G}$ alone. Also the $\tilde{M}$ is an irreducible, pre-smooth Zariski structure. In particular, $\tilde{M}$ admits quantifier elimination.

Outline. The proof involves using some nice choices of automorphisms of $\tilde{M}$ which can show that every existential formula can be reduced to a quantifier-free formula. One can then check all of the conditions exhaustively to show this structure is Zariski. A full proof can be found in [4].

An immediate corollary of this construction is the rather obvious lemma, which tells us that $\tilde{M}$ expands the automorphism group of $M$ to contain $\tilde{G}$. 
Lemma 5.3 (Automorphism Expansion Theorem). $\tilde{G}$ is a subgroup of $\operatorname{Aut}_{Z a r}(\tilde{M})$.

With these basic properties in mind, we can now turn some special attention to the finite subgroup $H$ we designated. In particular, this subgroup will give us a way of controlling the degree of reducibility for the expanded structure $\tilde{M}$.

Lemma 5.4 (Irreducible Expansions in Nonsplitting Case). Suppose H does not split in $\tilde{G}$ - that is, for every proper subgroup $G_{0}<\tilde{G}$

$$
G_{0} \cdot H \neq \tilde{G}
$$

Then every equidimensional Zariski expansion ' $\tilde{M}$ of $\tilde{M}$ is irreducible.

Outline. Every equidimensional expansion will induce an equidimensional cover of $M$ with $|H|$-many irreducible components. Every fiber has to intersect one component, so $H$ must act transitively on the set of components of the expansion. We note that only one of these components can exist or else the setwise stabilizer of the one component can be used with $H$ to contradict our splitting assumption.

For sake of brevity, we will omit the proof of the following lemma, but its proof can be found in [14]. This lemma will prove our theorem for us, and an interesting point to note about this is that our counterexamples from the lemma are objects constructed from classical algebro-geometric objects.

Lemma 5.5 (Non-Classical Rational and Elliptic Curves). Suppose $M$ is a rational curve or an elliptic curve over $K \vDash A C F_{0}$ where $H$ does not split, $\tilde{G}$ is nilpotent, and for some big integer $\mu$ there is a non-Abelian subgroup $G_{0} \leq \tilde{G}$ such that

$$
\left|\tilde{G}: G_{0}\right| \geq \mu
$$


Then $\tilde{M}$ is not interpretable in an algebraically closed field.

In other words, this lemma proves that the theory of Zariski structures is not completely reducible to classical algebraic geometry over algebraically closed fields by demonstrating that Zariski expansions can be obtained just from adding some obstructive Zariski properties to certain kinds og varieties. Therefore we've proved our theorem above, and the existence of such Zariski structures motivates a new dichotomy :

Definition 5.6 (Classical Zariski Structure). An irreducible pre-smooth Zariski structure $M$ which is interpretable in $A C F$ will be called a classical Zariski structure. Otherwise we call $M$ a nonclassical Zariski structure.

This kind of construction becomes more difficult to analyze when the dimension of $M$ is greater than 1, but there is a nice collection of tools which can be used to analyze some of these higher-dimensional situations in the case of varieties, which can still provide nice tools if one were to want to construct some of these more eccentric structures. Some additional theorems can be found in Chapter 5 of [14] - including some theorems for the case of Abelian and semi-Abelian varieties.

\subsection{Noncommutative Geometry for the Nonclassical Case}

With the existence of such nonclassical Zariski structures, we reframe our question : under what circumstances can be say that a Zariski structure is classical? To exposit all we know of this classification is a fairly arduous task, so here we will only present a class of example constructions directed at the application of these constructions towards physics. 
Our main principle will be to develop a kind of correspondence between Zariski structures and what we will consider quantum algebras. This construction will follow not only follow the analogy between classical and quantum (ie, nonclassical) mechanics, but the constructions presented here will provide our main source of algebraic objects for computation when applying our theory of Zariski structures to quantum mechanics. The key analogy will be that, in some sense, the classical setting of our Zariski structures will correspond to when the operators of our algebra commute, whereas the nonclassical cases will be precisely the cases where the commutation relations hold up to a nontrivial scalar multiple in our quantum algebras.

We will demonstrate the general method of construction by beginning with an arbitrary algebraically closed field $K$ and an algebra $\mathcal{A}$ over $K$ such that the following properties hold, and any algebra which satisfies these properties will be our notion of a quantum algebra:

(1.) $\mathcal{A}$ is an associative, unital affine $K$-algebra with generators $U_{1}, U_{2}, \ldots, U_{d}$ with relations defined from a finite set of parameters $C \subseteq K$. We also assume $\mathcal{A}$ is a finite-dimensional module over its central subalgebra $\mathcal{Z}=Z(\mathcal{A})$

(2.) The central subalgebra $\mathcal{Z}$ is a unital, finitely generated, commutative $K$-algebra without zero divisors. In other words, if $\operatorname{Max}(\mathcal{Z})$ is the set of maximal ideals of $\mathcal{Z}$, then $\operatorname{Max}(\mathcal{Z})$ can be identified with the $K$-points of an irreducible affine algebraic variety $V$ over $C$.

(3.) There is some $N \in \mathbb{Z}^{+}$such that for each $m \in \operatorname{Max}(\mathcal{Z})$ we can correspond $m$ to an $\mathcal{A}$-module $M_{m}$ of dimension $N$ over $K$ such that $m$ annihilates $M_{m}$. Furthermore, the isomorphism type of each of these modules is uniformily determined by a solution to a system of polynomial equations $P^{A}$ over $K$ and $V$, 
with variables $t_{i j k}$ as variables over $K$ and $m$ as a variable over $V$, such that for each $m \in V$ there is a set $T=\left\{t_{i j k}: i \leq d\right.$ and $\left.j, k \leq N\right\}$ satisfying $P^{A}(t, m)=0$ we can associate a basis $e_{1}, e_{2}, \ldots, e_{N}$ to the $K$-vector space of $M_{m}$ with the relation

$$
\bigwedge_{i \leq d ; j \leq N} U_{i}\left(e_{j}\right)=\sum_{k=1}^{N} t_{i j k} e_{k}
$$

Any basis satisfying these properties is called canonical.

(4.) There is a finite group $\Gamma$ and a map $g: V \times \Gamma \rightarrow \mathrm{GL}_{N}(K)$ such that, for each $\gamma \in \Gamma$, the map $g(\cdot, \gamma): V \rightarrow \mathrm{GL}_{N}(K)$ is rational and $C$-definable (that is, defined on an open subset of $V$ ). Also, for any $m \in V$ the domain of definition $\operatorname{Dom}_{m}$ for the map $g(m, \cdot): \Gamma \rightarrow \mathrm{GL}_{N}(K)$ is a subgroup of $\Gamma$. Furthermore $g(m, \cdot)$ is an injective homomorphism on its domain, and for any two canonical bases $e_{1}, e_{1}, \ldots, e_{N}$ and $e_{1}^{\prime}, e_{2}^{\prime}, \ldots, e_{N}^{\prime}$ of $M_{m}$ there is some $\lambda \in K^{\star}$ and $\gamma \in \operatorname{Dom}_{m}$ such that

$$
e_{i}^{\prime}=\lambda \sum_{1 \leq j \leq N} g_{i j}(m, \gamma) e_{j}
$$

Remark 5.7 (Some Explanation of these Assumptions). To give some brief explanation of these assumptions, and how they contribute to our analogy and constructions, we will first note that (1) tells us that our operator algebra is somehow generated from some finite-dimensional operator structure adjoined to some commutative algebra $\mathcal{Z}$, and whose operator relations are sufficiently representable using a finite set of parameters from the field $K$. When this assumption is conjoined with (2), the assumption becomes much more meaningful, since (2) says that our commutative algebra $\mathcal{Z}$ can be regarded as coming from a variety over $K$ - such as a coordinate ring of an affine variety. Thus (1) and (2) in conjunction say that our algebra $\mathcal{A}$ is constructed from adjoining some potentially noncommutative operator structure to 
an algebra coming from a classical kind of Zariski structure.

(3) tells us that the variety defined by the maximal spectrum of our classical algebra $\mathcal{Z}$ is equipped with a vector bundle whose fibers are all $\mathcal{A}$-modules consisting of elements that become trivialized when multiplied by $m$. Furthermore, the points $m$ on the variety correspond bijectively to the isomorphism types of their modules $M_{m}$, and two different points of the variety correspond to non-isomorphic modules. Furthermore, we think of each of these modules as having representations of each operator $U_{i}$ in terms of matrices consisting of the $t_{i j k}$ coefficients.

(4) tells us that there is a parameterized representation of a subgroups of a finite group $\Gamma$ in $K$ that rationally varies along the variety $V$. This representation faithfully represents the subgroup represented at each point, and the representations at each point correspond to the change-of-basis operators between canonical bases. Let $\Gamma_{m}=\operatorname{Dom}_{m}$ and let us denote $g(m, \gamma)$ for $\gamma \in \Gamma_{m}$ as simply $\gamma$ or possible $\gamma_{m}$ when the point of evaluation $m \in V$ is not clear from context.

With these rules specified for our algebra $\mathcal{A}$, and all of its related objects, we can now try to build a new Zariski structure from such an algebra.

Construction 5.8 (Associated Zariski Structure of a Quantum Algebra). Let $\mathcal{A}$ be a quantum algebra and let $V=V(\mathcal{A})$ stands for the $K$-points of the variety $\operatorname{Max}(\mathcal{Z})$ which can be treated as the set of $\mathcal{A}$-modules $M_{m}$ with $m \in \operatorname{Max}(\mathcal{Z})$. Begin by defining the disjoint union

$$
\tilde{V}=\bigcup_{m \in V} M_{m}
$$

Then, for each $m \in V$, choose a canonical basis $\mathcal{B}_{m}=\left\{e_{1}(m), e_{2}(m), \ldots, e_{N}(m)\right\}$ for $M_{m}$, and obtain all other canonical bases from actions by elements of $\Gamma_{m}$ : 


$$
E_{m}=\Gamma_{m} \mathcal{B}_{m}=\left\{\left(e_{1}^{\prime}(m), \ldots, e_{N}^{\prime}(m)\right): \exists \gamma \in \Gamma_{m} \text { such that } e_{i}^{\prime}(m)=\sum_{1 \leq j \leq N} \gamma_{i j} e_{j}(m)\right\}
$$

We also identify $V$ as its subset of $\mathbb{A}^{k}(K)$ for some $k \in \mathbb{Z}^{+}$and define the projection $\operatorname{map} \pi: \tilde{V} \rightarrow V$ by

$$
\pi(x)=m \quad \text { if } x \in M_{m}
$$

We then define the full language on the multi-sorted structure $(\tilde{V}, V, K)$ (which we will generally abbreviate by $\tilde{V}$ ) by the following relations

(1.) A ternary relation $S(x, y, z)$ such that $S(x, y, z)$ holds if and only if there is a $m \in V$ such that $x, y, z \in M_{m}$ and $x+y=z$

(2.) A ternary relation $a \cdot x=y$ defined for $a \in K$ and $x, y \in M_{m}$ as interpreting scalar multiplication by $a$ in the module $M_{m}$.

(3.) Binary relations $U_{i} x=y$ for each operator $U_{i}$, which for each $x, y \in M_{m}$ interprets the action of $U_{i}$ on the module $M_{m}$.

(4.) Relations $E_{m} \subseteq V \times \tilde{V}^{N}$ with $E(m, \mathcal{B})$ interpreted as $\mathcal{B} \in E_{m}$ ( $E$ defines a predicate for identifying canonical bases at each point ).

Finally, we also assume that the sort $V$ is endowed with its Zariski structure as a variety. With all of this, we say that $(\tilde{V}, V, K)$ is the associated Zariski struture of the quantum algebra $\mathcal{A}$ in the full language. If we omit (4) from our language (the canonical bases predicate), then we obtain $(\tilde{V}, V, K)$ endowed with the weak language over $\mathcal{A}$.

Remark 5.9. We note that the sorts $V$ and $K$ are bi-interpretable over $C$, and that the parametered representation $g$ of $\Gamma$ in the quantum algebra is definable in the weak language of $\tilde{V}$ since it is rational. 
Definition $5.10(\operatorname{Th}(\mathcal{A}-\bmod ))$. The first order theory $\operatorname{Th}(\mathcal{A}-\bmod )$ describing $(\tilde{V}, V, K)$ is the set of axioms described by

(1.) $K$ is algebraically closed with characteristic $p$, and $V$ is the Zariski structure on the $K$-points of the variety $\operatorname{Max}(\mathcal{Z})$.

(2.) For each $m \in V$, the action of scalars of $K$ on $U_{1}, \ldots, U_{d}$ defines the structure of an $\mathcal{A}$-module of dimension $N$ on $\pi^{-1}(m)$.

(3.) Assumption 3 about quantum algebras holds for the given $P^{A}$.

(4.) For the parameterzied representation $g: V \times \Gamma \rightarrow \mathrm{GL}_{N}(K)$ given by assumption 4 about quantum algebras, and any pair of canonical bases $\mathcal{B}=\left\{e_{1}, \ldots, e_{N}\right\}$ and $\mathcal{B}^{\prime}=\left\{e_{1}^{\prime}, \ldots, e_{N}^{\prime}\right\}$ in $E_{m}$, there is a $\gamma \in \Gamma$ such that

$$
e_{i}^{\prime}=\sum_{1 \leq j \leq N} g_{i j}(m, \gamma) e_{j}
$$

Moreover, $E_{m}$ is an orbit under the action $\Gamma_{m}$.

Here we will give two examples of these kinds of quantum algebras and their associated Zariski structures to demonstate some of our assertions about these algebras and the supposed analogy with different kinds of physical mechanics.

Example 5.11 (Trivial Line Bundle on Affine Variety). Let $\mathcal{A}$ be a commutative unital affine $K$-algebra. Thus $\mathcal{Z}=\mathcal{A}$, and so $V=\operatorname{Max}(\mathcal{Z})=\operatorname{Max}(\mathcal{A})$ is the corresponding affine variety. The maximal ideals $m \in \operatorname{Max}(\mathcal{A})$ will annilate irreducible $\mathcal{A}$-modules $M_{m}$ which are all one-dimensional as $K$ vector spaces. This gives us that $\tilde{V}$ is a trivial line bundle of each $M_{m}$ over their respective points in $V$. Since the bundle is trivial, it will be definable over $K$ and will have a global section which is a rational map

$$
s: V \rightarrow \tilde{V}
$$


where each $s(m)$ is a canonical basis of $M_{m}$ for each $m \in V$, and each $\Gamma_{m}$ is the trivial group for all $m$. In this sense, this object is a classical Zariski structure since all components of its structure are reducible to algebraic geometry over $K$.

Example 5.12 (Quantum 2-Torus at Roots of Unity). Let $\epsilon$ be an $\ell$-th root of unity in $K$ and let $\mathcal{A}$ now be the quantum algebra generated by $\mathbf{U}, \mathbf{U}^{-1}, \mathbf{V}, \mathbf{V}^{-1}$ satisfying the following relations

$$
\mathbf{U U}^{-1}=\mathbf{V V}^{-1}=1 \text { and } \mathbf{U V}=\epsilon \mathbf{V U}
$$

We will denote this algebra $T_{\epsilon}^{2}=\mathcal{A}$.

Note that the center $\mathcal{Z}$ of $T_{\epsilon}^{2}$ is generated by $\mathbf{U}^{\ell}, \mathbf{U}^{-\ell}, \mathbf{V}^{\ell}, \mathbf{V}^{-\ell}$ and that the variety $V=\operatorname{Max}(\mathcal{Z})$ is isomorphic to $K^{*} \times K^{*}$.

Any irreducible $T_{\epsilon}^{2}$-module $M$ will be a $K$-vector space with dimension $N=\ell$, and with a basis given by the space of $\mathbf{U}$-eigenvectors $\left\{e_{0}, e_{1}, \ldots, e_{\ell-1}\right\}$ satsifying for eigenvalues $\mu$ of $\mathbf{U}$ and $\nu$ of $\mathbf{V}$

$$
\begin{aligned}
& \mathbf{U} e_{i}=\mu \epsilon^{i} e_{i} \\
& \mathbf{V} e_{i}= \begin{cases}\nu e_{i+1} & \text { if } i<\ell-1 \\
\nu e_{0} & \text { if } i=\ell-1\end{cases}
\end{aligned}
$$

We also have a basis of $\mathbf{V}$ eigenvectors $\left\{g_{0}, \ldots, g_{\ell-1}\right\}$ satisfying the change of basis relation

$$
g_{i}=e_{0}+\epsilon^{i} e_{1}+\ldots+\epsilon^{i(\ell-1)} e_{\ell-1}
$$

and therefore 


$$
\begin{aligned}
& \mathbf{V} g_{i}=\nu \epsilon^{i} g_{i} \\
& \mathbf{U} g_{i}= \begin{cases}\mu g_{i+1} & \text { if } i<\ell-1 \\
\mu g_{0} & \text { if } i=\ell-1\end{cases}
\end{aligned}
$$

We can let $a=\mu^{\ell}$ and let $b=\nu^{\ell}$. Then $\left(\mathbf{U}^{\ell}-a\right)$ and $\left(\mathbf{V}^{\ell}-b\right)$ are generators for $\operatorname{Ann}(M)$. The module is determined uniquely once $a$ and $b$ are given. Therefore $V$ is isomorphic to the two-dimensional torus $K^{*} \times K^{*}$. Furthermore, the coefficients for the matrix representations

$$
T=\left\{t_{i j k}: i \leq d \text { and } j, k \leq \ell\right\}
$$

for the operators $\mathbf{U}$ and $\mathbf{V}$ are determined by $\mu$ and $\nu$ satisfying the polynomial equations

$$
\mu^{\ell}=a \text { and } \nu^{\ell}=b
$$

With respect to this basis, the group $\Gamma_{m}=\Gamma$ becomes the nilpotent group of order $\ell^{3}$ generated by the matrices

$$
\left(\begin{array}{ccccc}
0 & 1 & 0 & \ldots & 0 \\
0 & 0 & 1 & \ldots & \vdots \\
0 & 0 & 0 & \ldots & \vdots \\
\vdots & \vdots & \vdots & \ddots & 1 \\
1 & 0 & 0 & \ldots & 0
\end{array}\right) \text { and }\left(\begin{array}{ccccc}
1 & 0 & 0 & \ldots & 0 \\
0 & \epsilon & 0 & \ldots & \vdots \\
0 & 0 & \epsilon^{2} & \ldots & \vdots \\
\vdots & \vdots & \vdots & \ddots & \vdots \\
0 & \ldots & \ldots & \ldots & e^{\ell-1}
\end{array}\right)
$$

representing the operators $\mathbf{U}$ and $\mathbf{V}$ in the eigenbases we used to define the canonical bases. This associated Zariski structure $\tilde{V}\left(T_{\epsilon}^{2}\right)$ to the quantum algebra $T_{\epsilon}^{2}$ defined 
by this construction is called the quantum 2-torus.

We will remark without proof the following theorem whose proof can be found in [14] in Chapter 5, Section 3, derived from a general case proven for quantum $n$-torus. Proposition 5.13 (Quantum Tori are (generally) Nonclassical). The associated Zariski structure $\tilde{V}\left(T_{\epsilon}^{2}\right)$ is not interpretable in any algebraically closed field for any $\epsilon \neq 1$.

There will be a generalization of quantum 2-tori can be defined for any $\epsilon \in K^{*}$, but the resulting structure may not always be a Noetherian Zariski structure (as opposed to a different kind of Zariski structure which are often called analytic Zariski structures, which are discussed in chapter 6 of [14]). This kind of torus will be presented in the next chapter. For now, one idea to note about quantum tori at roots of unity is that we can imagine the root $\epsilon=\exp \left(2 \pi i \frac{m}{n}\right)$ for some $m, n \in \mathbb{Z}$. We can then ask whether or not there is an appropriate way to turn the rational number $\frac{m}{n}$ into a convergent sequence of rational numbers to approximate an aribtary real number, and let the limiting process represent an approximation of structures. Before we do this however, we will end this chapter with the following properties which demonstrate the logical strength of these kinds of Zariski structures.

Proposition 5.14 (Isomorphism Lifting to Associated Structures). Let $\tilde{V}_{1}$ and $\tilde{V}_{2}$ be associated Zariski structures of $\mathcal{A}$ in the weak (or full) language with the same $P^{A}$ system of polynomial equations over the field $K$. Then the natural isomorpism $i: V_{1} \cup K \rightarrow V_{2} \cup K$ over $C$ can be lifted to a Zariski isomorphism

$$
\tilde{i}: \tilde{V}_{1} \rightarrow \tilde{V}_{2}
$$

Proof. We can assume that the natural isomorphism between $i: V_{1} \cup K \rightarrow V_{1} \cup K$ is the identity map on $V \cup K$ without loss of generality. 
We know that $\pi_{1}^{-1}(m)$ and $\pi_{2}^{-1}(m)$ each have the structure of a module for each $m \in V$. We can then build an isomorphism between $\pi_{1}^{-1}(m)$ and $\pi_{2}^{-1}(m)$ by assumption (3) about quantum algebras that at each $m$ we can find $T=\left\{t_{i j k} \epsilon\right.$ $K: i \leq d$ and $j, k \leq N\}$ solving the polynomial equations $P^{A}$ and choose for each such solution pair of canonical bases $\left\{e_{1}^{(1)}(m), e_{2}^{(1)}(m), \ldots, e_{N}^{(1)}(m)\right\}$ for $\pi_{1}^{-1}(m)$ and $\left\{e_{1}^{(2)}(m), e_{2}^{(2)}(m), \ldots, e_{N}^{(2)}(m)\right\}$ for $\pi_{2}^{-1}(m)$ which can be used to represent each operator $U_{i}$ in terms of the basis using coefficients from $T$. This means the relationship will be preserved if we extend our map $i$ by the mapping

$$
i_{m}: \sum_{1 \leq j \leq N} z_{j} e_{j}^{(1)}(m) \mapsto \sum_{1 \leq j \leq N} z_{j} e_{j}^{(2)}(m)
$$

for any choice of $z_{1}, z_{2}, \ldots, z_{N} \in K$. This induces an isomorphism $i_{m}: \pi_{1}^{-1}(m) \rightarrow$ $\pi_{2}^{-1}(m)$. Therefore we can build the isomorphism

$$
\tilde{i}: \tilde{V}_{1} \rightarrow \tilde{V}_{2} \text { defined by } \tilde{i}=\bigcup_{m \in V} i_{m}
$$

This proves that there is an isomorphism between $\tilde{V}_{1}$ and $\tilde{V}_{2}$ under the weak language. To prove the full language case, note that the maps $i_{m}$ will also preserve the orbit relation between any two canonical bases in $E_{m}$, and so they will preserve the structure of the full language.

An immediate corollary of this result is the following theorem.

Theorem 5.15 (Categoricity of Associated Structures of Quantum Algebrass). For any quantum algebra $\mathcal{A}$, the theory $\operatorname{Th}(\mathcal{A}$-mod $)$ is uncountably categorical in both the full and weak languages. 
The importance of this theorem for physical application can perhaps be roughly interpreted as saying there is only one model of the associated Zariski structure of a quantum algebra, which as we hope we can modify to represent constructs such as operator algebras of observables in quantum mechanics. There is plenty more nice results about these kinds of nonclassical Zariski geometries which were originally developed in [12] and represented in [14]. Most of these facts are deeper modeltheoretic results about Zariski structures, such as properties regarding how certain formulas can be simplified modulo the theories of these Zariski structures, but we will omit these and leave them to the curiousity of the reader.

We note that our main examples have consisted of building operator structures over some classical geometries and realizing that these additional structures seem to be reducible to classical structures precisely in the cases where the operators commute, but will induce nonclassical geometric objects in the. This can be seen as analogous to the idea that quantum mechanical operators commute in precisely the setting of classical mechanics, but their commutators are essentially just a nontrivial scaling operation. In this sense, this motivates our eagerness to label our structures built here as quantum structures, since these methods can be seen as a kind of quantization of classical Zariski structures. With this being said, let us turn our attention towards applying our theory of Zariski structures to quantum mechanics. 


\section{CHAPTER 6}

\section{APPLICATIONS TO QUANTUM MECHANICS}

Now that we have demonstrated some of the basic methodological principles of nonclassical Zariskis structures, let us now turn more explicitly towards applying these constructs to quantum mechanics. In particular, we will operate under the following principle : there is a kind of semantic-syntactic correspondence which we would like to utilize between our notion of quantum algebra, and our notion of associated Zariski structure. A quantum algebra essentially prescribes some kind of algebraic rules pertaining to how some collection of operators works on some vectors spaces. We then create a canonical way of associating that algebra with some kind

of geometrical space which encodes the behavior of the algebra on its elements. One could compare this correspondence principle we used as somewhat analogous to the correspondence of finite-dimensional Lie algebras to some kind of natural choice of matrix Lie group by Lie's third theorem.

\subsection{Generalizing Quantum Tori}

First, let generalize the notion of quantum 2-torus that we used before. In particular, we would like our operators $\mathbf{U}$ and $\mathbf{V}$ to eventually represent the position and momentum operators of quantum mechanics in one-dimension. With that being said, a fundamental component of the mathematical structures used in quantum mechanics 
is the inner product, which allows us to compute the probability distributions of the observable quantities associated with these operator. After we develop the newer quantum torus definition from [13], we will then develop our notion of structural approximation based on ultraproducts, and then apply these two tools to physics to demonstrate a way these tools can help physicists avoid the process of renormalization in an example of computing a Feynman propagator.

Construction 6.1 (Generalized Quantum 2-torus). Let $K$ be an algebraically closed field, and let $\mathcal{A}_{\epsilon}$ be the $K$-algebra generated by the operators $\mathbf{U}, \mathbf{U}^{-1}, \mathbf{V}$, and $\mathbf{V}^{-1}$ satisfying the relations

$$
\mathbf{U U}^{-1}=\mathbf{V V}^{-1}=1 \text { and } \mathbf{V U}=\epsilon \mathbf{U V}
$$

where $\epsilon \in K^{*}$. Recall that $V=\operatorname{Max}(\mathcal{Z})$ is isomorphic to $K^{*} \times K^{*}$ under these assumptions. Furthermore, let us note that

$$
\mathcal{G}=\left\{\epsilon^{m}: m \in \mathbb{Z}\right\}
$$

is a multiplicative subgroup of $K^{*} \times K^{*}$.

Now for each $(u, v) \in K^{*} \times K^{*}$, let us construct a pair of $\mathcal{A}_{\epsilon}$-modules denoted $M_{|u, v\rangle}$ and $M_{\langle v, u|}$. In the case where $\epsilon$ is a root on unity as before, $M_{|u, v\rangle}$ will be isomorphic to $M_{\langle v, u|}$.

In this case, our canonical bases can be infinitary, and they will be labelled by the notation $\mathcal{B}_{|u, v\rangle}=\{\mathbf{u}(g u, v): g \in \mathcal{G}\}$ to represent the eigenvectors of $\mathbf{U}$ with their corresponding eigenvalues $g \in \mathcal{G}$. In the case of the a root of unity (in which $\mathcal{G}$ is torsion), we note that our basis will reduce to a finite one. We will now interpret the 
operator $\mathbf{U}$ as a linear operator on $M_{|u, v\rangle}$ defined by its action on the canonical basis

$$
\begin{aligned}
\mathbf{U}: \mathbf{u}(g u, v) & \mapsto g u \cdot \mathbf{u}(g u, v) \\
\mathbf{V}: \mathbf{u}(g u, v) & \mapsto v \cdot \mathbf{u}\left(\left(\epsilon^{-1} g\right) u, v\right)
\end{aligned}
$$

Dually, we interpret the operators on $M_{\langle v, u|}$ by their actions on the canonical eigenbasis $B_{\langle v, u|}=\{\mathbf{v}(g v, u): g \in \mathcal{G}\}$ by

$$
\begin{aligned}
& \mathbf{U}: \mathbf{v}(g v, u) \mapsto u \cdot \mathbf{v}\left(\left(\epsilon^{-1} g\right) v, u\right) \\
& \mathbf{V}: \mathbf{v}(g v, u) \mapsto g v \cdot \mathbf{v}(g v, u)
\end{aligned}
$$

We would like to identify these modules as submodules of some common ambient module, but for now we will just note that when $\epsilon$ is a root of unity, we should expect these two modules to be the same modulo some change of basis.

Let $K^{*} / \mathcal{G}$ be the cosets of $K^{*}$ by $\mathcal{G}$. Define some choice function $\rho: K^{*} / \mathcal{G} \rightarrow K^{*}$ which chooses a representative from each coset, and let $R$ be the range of this choice function, and so it is a set consisting of precisely one representative from each coset. Let

$$
\begin{aligned}
& \mathbb{U}_{\rho}=\left\{g_{1} \cdot \mathbf{u}\left(g_{2} u, v\right):(u, v) \in R^{2} \text { and } g_{1}, g_{2} \in \mathcal{G}\right\} \subseteq \bigcup_{u, v \in K^{*}} M_{|u, v\rangle} \\
& \mathbb{V}_{\rho}=\left\{g_{1} \cdot \mathbf{v}\left(g_{2} v, u\right):(u, v) \in R^{2} \text { and } g_{1}, g_{2} \in \mathcal{G}\right\} \subseteq \bigcup_{u, v \in K^{*}} M_{\langle v, u|}
\end{aligned}
$$

We can now add the predicates $\mathbb{U}$ and $\mathbb{V}$ as unary predicates designating the sorts $\mathbb{U}_{\rho}$ and $\mathbb{V} \rho$ respectively. We will now consider the actions on each of the sorts

$$
K \mathbb{U}_{\rho}=\left\{x \cdot \mathbf{u}(g u, v):(u, v) \in R^{2} \text { and } g \in \mathcal{G} \text { and } x \in K\right\}
$$




$$
K \mathbb{V}_{\rho}=\left\{y \cdot \mathbf{v}(g v, u):(u, v) \in R^{2} \text { and } g \in \mathcal{G} \text { and } y \in K\right\}
$$

We now consider a pairing to be a ternary relation representing a function

$$
\langle\cdot \mid \cdot\rangle: \mathbb{U}_{\rho} \times \mathbb{V}_{\rho} \rightarrow K
$$

defined as such : for any $(u, v) \in K^{*} \times K^{*}$ and any $\epsilon^{s} \mathbf{v}\left(\epsilon^{m} v, u\right) \in \mathbb{V}_{\rho}$ and $\epsilon^{r} \mathbf{u}\left(\epsilon^{k} u, v\right) \in \mathbb{U}_{\rho}$ we set

$$
\left\langle\epsilon^{s} \mathbf{v}\left(\epsilon^{m} v, u\right) \mid \epsilon^{r} \mathbf{u}\left(\epsilon^{k} u, v\right)\right\rangle=\epsilon^{-k m-s+r}
$$

and we leave the pairing undefined for any situation $\left\langle\epsilon^{s} \mathbf{v}\left(v^{\prime}, u\right) \mid \epsilon^{r} \mathbf{u}\left(u^{\prime}, v\right)\right\rangle$ where $v^{\prime} \notin$ $v \cdot \mathcal{G}$ or $u^{\prime} \notin u \cdot \mathcal{G}$

The triple $\left(\mathbb{U}_{\rho}, \mathbb{V}_{\rho}, K\right)$ with the structure of a field on $K$, the operators $\mathbf{U}$ and V acting on the sorts as described above, and the pairing function between the two sorts will be the structure of the (geometric) quantum 2-torus associated with $\mathcal{A}_{\epsilon}$. This finishes our construction.

With this construction in mind, we claim that the technique of proof which was used in [14] to prove that our previous notion of quantum tori are nonclassical Zariski geometries, and that constructions built from these techniques are uncountably categorical in their theories, one just needs to perform a simple adaption of those proofs. We summarize the results in the following theorem.

Theorem 6.2 (Classification Results of Generalized Tori). Given an algebraically closed field $K$ and some $\epsilon \in K$, then for any two choice functions $\rho_{1}$ and $\rho_{2}$ from $K^{*} / \mathcal{G}$ to $K^{*}$ and the two corresponding quantum tori $\left(\mathbb{U}_{\rho_{1}}, \mathbb{V}_{\rho_{1}}, K\right)$ and $\left(\mathbb{U}_{\rho_{2}}, \mathbb{V}_{\rho_{2}}, K\right)$, there is an isomorphism over $K$ between the two structures. 
If $\epsilon$ is a root of unity, then the quantum torus will be a Noetherian Zariski structure. (ie, nonanalytic Zariski structure). Furthermore, if $\epsilon \neq 1$, then the torus is not definable in $(K, \mathcal{G})$, the field $K$ adjoined with a predicate for defining the multipicative subgroup $\mathcal{G}$.

With these results in mind, let us now define the inner product on this structure in the situation where the $\epsilon$ is a root of unity. Of course, we now will assume that $K=\mathbb{C}$ so the inner-product can be well-defined.

Construction 6.3 (Inner Product on 2-torus at Roots of Unity). Let $\left(\mathbb{U}_{\rho}, \mathbb{V}_{\rho}, \mathbb{C}\right)$ be a quantum torus corresponding to $\mathcal{A}_{\epsilon}$ with $\epsilon$ a root of unity of order $N$. We define our inner-product on $M_{(u, v)}=M_{|u, v\rangle}=M_{\langle v, u|}$ by the map $(\cdot \mid \cdot): M_{(u, v)} \times M_{(u, v)} \rightarrow \mathbb{C}$ by inducing a sequilinear map on the following basis evaluations

$$
\begin{aligned}
& \left(\mathbf{u}\left(\epsilon^{k} u, v\right) \mid \mathbf{v}\left(\epsilon^{m} v, u\right)\right)=\frac{1}{\sqrt{N}}\left\langle\mathbf{v}\left(\epsilon^{m} v, u\right) \mid \mathbf{u}\left(\epsilon^{k} u, v\right)\right\rangle=\frac{\epsilon^{-k m}}{\sqrt{N}} \\
& \left(\mathbf{u}\left(\epsilon^{k} u, v\right) \mid \mathbf{u}\left(\epsilon^{m} u, v\right)\right)=\delta_{k m} \\
& \left(\mathbf{v}\left(\epsilon^{k} v, u\right) \mid \mathbf{v}\left(\epsilon^{m} v, u\right)\right)=\delta_{k m}
\end{aligned}
$$

where $\delta_{k m}$ denotes the Kronecker delta function. Note that these rules tell us that our canonical bases are defined as orthonormal, and that the transition matrices between them can be given by

$$
\begin{aligned}
\mathbf{u}\left(\epsilon^{k} u, v\right) & =\frac{1}{\sqrt{N}} \sum_{m=0}^{N-1} \epsilon^{-k m} \mathbf{v}\left(\epsilon^{m} v, u\right) \\
\mathbf{v}\left(\epsilon^{m} u, v\right) & =\frac{1}{\sqrt{N}} \sum_{k=0}^{N-1} \epsilon^{k m} \mathbf{v}\left(\epsilon^{k} u, v\right)
\end{aligned}
$$




\subsection{Structural Approximation}

Now that we have a notion of inner product defined, we have now adapted the tools of our nonclassical geometries to suit our needs. In particular, let us not that if we were to let $\epsilon=\exp (2 \pi i \hbar)$ where $\hbar$ denotes the reduced Planck constant of physics, and we take $\mathbf{U}=\exp (i P)$ and $\mathbf{V}=\exp (2 \pi i Q)$ for $P$ the momentum operator and $Q$ the position operator satisfying the Heisenberg commutation relations

$$
Q P-P Q=[Q, P]=i \hbar I
$$

then the operators $\mathbf{U}$ and $\mathbf{V}$ will form a quantum torus assuming they follow the Weyl commutation relations

$$
\mathbf{V U}=\epsilon \mathbf{U V}
$$

which corresponds precisely with the commutation relation (6.1) that we used to define our quantum algebras. We will remark more on this later, but first let us introduce the notion of structural approximation.

Definition 6.4 (Structural Approximation). Let $M$ be a topological structure, and let $\left(M_{i}: i \in I\right)$ be a family of structures each under the same language as $M$. We say that $M$ is structurally approximated by $\left(M_{i}: i \in I\right)$ along an ultrafilter $\mathcal{U}$ on $I$ if for some elementary extension of the ultraproduct ${ }^{*} M \geqslant \prod M_{i} / \mathcal{U}$ there is a surjective homomorphism $\lim _{\mathcal{U}}:{ }^{\star} M \rightarrow M$

Remark 6.5 (Different Approximation Notions). In a more recent draft written by Zilber, there is a distinction made between so-called syntactic approximation and semantic approximation as kinds of approximations. In our case, the notion of structural approximation means precisely the same thing as semantic approxima- 
tion. Furthermore, in the case of quasi-compact structures, the notions of syntactic approximation and semantic approximation become equivalent.

Notation 6.6 (Use of Limit Function). Sometimes when we have a family of structures $\left\{M_{i}: i \in I\right\}$ which we know approximate $M$ under some ultrafilter $\mathcal{U}$, we will sometimes skip writing out the ultraproduct (or the extension of the ultraproduct used in the approximation) and simply say that

$$
M=\lim _{\mathcal{U}} M_{i}
$$

if the sequence of structures approximates $M$.

To get a grasp of the properties of structural approximation, let us look at some examples of how structural approximation pertains to some of the topological structure notions we discussed. In particular, we can derive the following theorem which can sometimes give an intution about what kinds of structures result from this approximation.

Proposition 6.7 (Quasi-Compact Approximations). Let $M$ be a topological structure approximated by $\left(M_{i}: i \in I\right)$ over an ultrafilter $\mathcal{U}$, where $M_{i}=M$ for each $i \in I$. If the elementary extension ${ }^{*} M \geqslant \prod M_{i} / \mathcal{U}$ which surjectively maps onto $M$ is saturated, then $M$ must be quasi-compact.

Proof. First, note that for each $a \in M$ the singleton closed set of that point is precisely defined by a positive formula that expresses that $a$ is the only element of $M$ in that singleton set. We can then choose a point $\hat{a} \in M^{D}$ for each $a$ such that uniquely $\lim _{\mathcal{U}}(\hat{a})=a$ by surjectivity (if this point wasn't unique, then the surjective map wouldn't preserve the statement that this set consists uniquely of one element). So 
we can map $i: a \mapsto \hat{a}$ as an embedding of $M$ into $M^{D}$. This can be shown to mean that $M^{D}$ is a total specialization onto $M$, and therefore by Proposition 3.15, we know $M$ must be quasi-compact.

This proposition indicates a theme about structural approximation that we will use to our advantage when studying their application to physics : structural approximation is well-studied for approximating quasi-compact structures. This makes the structural approximation somewhat reminiscent of notions like the Gromov-Hausdorff limit of a family of compact metric spaces. With this in mind, one can actually prove that the Gromov-Hausdorff limit can be understood as an instance of structural approximation under an appropriate choice of language for metric spaces, which is proven in [15].

We also get the next result, which will be useful for our particular case for what it says about approximation in the class of compactified cyclic groups. Particularly, consider the following construction.

Construction 6.8 (Two-End Compactifications of $\mathbb{Z}$ and $\mathbb{Z} / n \mathbb{Z}$ ). Let $\mathbb{Z}$ have the additive integer group structure and let $\overline{\mathbb{Z}}=\mathbb{Z} \cup\{\infty,-\infty\}$ with the ternary relation $x+y=z$ intepreted on $\mathbb{Z}$ as addition, but interpreted for infinities as

$$
\begin{array}{r}
x+(-\infty)=-\infty \text { for all } x \in \mathbb{Z} \\
x+\infty=\infty \text { for all } x \in \mathbb{Z} \\
\infty+(-\infty)=x \text { for all } x \in \overline{\mathbb{Z}}
\end{array}
$$

Then we let $\mathcal{C}$ consist of all relations of the form $x+y=z$, the unary relations $x=n$ for each $n \in \overline{\mathbb{Z}}$, and all positive existential formulas generated in this language. We 
note that $(\overline{\mathbb{Z}}, \mathcal{C})$ is quasi-compact, and that this same language for compactification can also be applied to $\mathbb{Z} / n \mathbb{Z}$ for each $n \in \mathbb{Z}$. Let us write the compactification of $\mathbb{Z} / n \mathbb{Z}$ as $\overline{\mathbb{Z}} / n \overline{\mathbb{Z}}$

We now say that an ultrafilter $\mathcal{U}$ of subsets of $\mathbb{Z}^{+}$is profinite if for every $m \in \mathbb{Z}^{+}$ the subset $m \mathbb{Z}^{+}$is in $\mathcal{U}$, and we can state the following fact which will be useful for us in doing our physics approximations.

Proposition 6.9 (Compact Approximation of Cyclic Groups). Given an ultrafilter $\mathcal{U}$ of $\mathbb{Z}^{+}$, we have $\overline{\mathbb{Z}}=\lim _{\mathcal{U}} \overline{\mathbb{Z}} / n \overline{\mathbb{Z}}$ if and only if $\mathcal{U}$ is profinite.

Once again, the proof for this proposition can be found in [15], but for our purposes we will simply state the theorem and notes its uses in our situation.

In particular, with these structural approximation tools in place, we now note that we can work in the compactification of the quantum torus $(\overline{\mathbb{U}}, \overline{\mathbb{V}}, \overline{\mathbb{C}})$ instead. In this compactified structure, we let $\overline{\mathbb{C}}$ be the usual one-point compactification $\overline{\mathbb{C}}=\mathbb{C} \cup\{\infty\}$, and we let $\overline{\mathbb{U}}$ and $\overline{\mathbb{V}}$ be our compactifications determined by all the other structure whenever we compactify our group $\mathcal{G}$, which we note is isomorphic to $\mathbb{Z} / n \mathbb{Z}$ whenever $\epsilon$ is a root of unity, and is isomorphic to $\mathbb{Z}$ otherwise. That means that $\mathcal{G}$ can be compactified using the two-end compactification above.

Furthermore, we note that if we let $\left\{\epsilon_{n}\right\}_{n=1}^{\infty}$ be a sequence of roots of unity of the form $\exp \left(2 \pi i \frac{m_{n}}{n}\right)$, and we let $\frac{m_{n}}{n}$ metrically converge to $\hbar$, then we can structurally approximate the quantum 2-torus which models our Weyl commutation relations using our quantum tori at roots of unity. In fact, the argument for the proposition about compactifications of cyclic groups can be adapted to this scenario to prove the following theorem (assuming that some appropriate structure has been added to the group to define the metric within the structure). 
Theorem 6.10 (Approximation by Tori at Roots of Unity). A quantum torus at $\epsilon$ is approximated by a sequence of quantum tori at roots of unity $\exp \left(2 \pi i \frac{m_{n}}{n}\right)$ for $n \in \mathbb{Z}^{+}$

if and only if the ultrafilter of the approximation $\mathcal{U}$ is profinite and $\frac{m_{n}}{n}$ metrically converges to $\epsilon$ along $\mathcal{U}$.

We now have our geometries and approximation schemes set up, and now we will end our discussion with an application of these tools to computing some quantities from quantum mechanics which are often computed heuristically.

\subsection{Dirac Calculus and Applying the Torus}

We will now survery some of the proposed applications by Zilber in [13], which aims to provide an alternative way to rigorously compute some quantities in physics using these techniques in model theory; and constitutes a general project that Zilber has pursued seemingly beginning with his work on constructing examples of nonclassical Zariski geometries in [12].

To give a brief background, the Dirac calculus refers to a method that initially developed as a heuristic for calculating certain transformations of wave functions by operators expressed in terms of the position $Q$ and momentum $P$ operators satisfying the Heisenberg commutation relations. Using this method, we represent our wave functions as elements of some Hilbert space, expressed in terms of the eigenvectors of $Q$ (or, we could express the space in terms of eigenvectors of $P$ by Fourier duality). We then consider the time evolution operator of the quantum mechanical system given by

$$
K^{t}=e^{-i t \frac{H}{\hbar}}
$$


where $H$ is the Hamiltonian operator of the quantum mechanical system, which is normally a self-adjoint operator given by some

$$
H=\frac{P^{2}}{2 \hbar}+V(Q)
$$

where $V(Q)$ is the potential function with respect to position $Q$, and is often expressed as a polynomial.

The choice of $V(Q)$ essentially characterizes the particle under consideration, and the interactions of the particle will be determined by computing the time-evolution operator of the particle for its wave function, which is done by taking an integral. The only hiccup one encounters is that not all of these integrals converge, but due to the use of heuristic methods, physicists have still been able to find some ways to evaluate these integrals which still provides results that accurately predict physical phenomena.

The Hilbert space specified can usually be regarded in analogy to the Hamiltonian state space of classsical mechanics which is a 2-space with coordinates encoding onedimensional position and momentum of a particle, particularly with respect to the fact that the Hilbert space has a pair of mutually dual bases representing the eigenvectors of the position and momentum operators. In either mechanics, the spaces consist of all the different physical states of the particle. It is this quantum state space from which we would like to extract a Zariski structure.

Recall that if we let $\mathbf{V}^{b}=\exp (b i P)$ and $\mathbf{U}^{a}=\exp (2 a \pi i Q)$ for $a=1$ and $b=$ 1 (although we could assign $a$ and $b$ to be any rationals to obtain different kinds of operators), then we get that $\mathbf{V}^{b}$ and $\mathbf{U}^{a}$ will satisfy the following commutation relations by the Baker-Campbell-Hausdorff formula from Lie theory 


$$
\mathbf{V}^{b} \mathbf{U}^{a}=\epsilon^{a b} \mathbf{U}^{a} \mathbf{V}^{b} \text { where } \epsilon^{a b}=\exp (2 a b \pi i \hbar)
$$

Furthrmore, the eigenvectors $|p\rangle$ of $P$ corresponding to eigenvalue $p$ will be eigenvalues of $\mathbf{V}$ corresponding to eigenvalue $\exp ($ bip $)$, and the analogous result holds for the eigenvectors $|x\rangle$ of $Q$ for $\mathbf{U}$ with eigenvalue $\exp (2 a \pi i x)$. This use of bra-ket notation for one-dimensional quantum mechanics represents an eigenvector by its eigenvalue inside the ket brackets. For example, the eigenvector $|x\rangle$ of the position operator $Q$ will be a wave function whose probability distribution guarantees with probability 1 that a particle in the state represented by $|x\rangle$ is located at the position $x$ - the eigenvalue of the wave function.

In particular, by our theorem for generalized quantum tori, the $\left(\mathbf{U}^{a}, \mathbf{V}^{b}\right)$-system will form a Zariski structure. In particular, we can take the following maps

$$
\begin{aligned}
& \exp ^{a, b}:|x\rangle \mapsto \mathbf{u}\left(e^{2 a \pi \lambda_{x}}, 1\right) \\
& \exp ^{a, b}:|p\rangle \mapsto \mathbf{v}\left(e^{b \lambda_{p}}, 1\right)
\end{aligned}
$$

from the Hilbert space $\mathcal{H}$ to the respective eigenvectors of the $\left(\mathbf{U}^{a}, \mathbf{V}^{b}\right)$-system. We can then treat $|x\rangle$ and $|p\rangle$ as the limits of $\mathbf{u}\left(e^{2 a \pi x}, 1\right)$ and $\mathbf{v}\left(e^{b p}, 1\right)$ respectively when we let $a$ and $b$ both converge to 0 . This will pass our Dirac calculus problem to a structural approximation of Zariski structures problem, which we can avoid cases where Dirac calculus is ill-posed. From here forward, let us use the notation $\mathbf{U}=\mathbf{U}^{1}$ and $\mathbf{V}=\mathbf{V}^{1}$

We now will compute a time-evolution operator in the Zariski structure setting. We will start by expanding the $\left(\mathbf{U}^{a}, \mathbf{V}^{b}\right)$-system by a time-evolution operator $K^{t}$ for some choice of a Hamiltonian operator $H$. In particular, we will start with the 
Hamiltonian of a free particle, whose potential $V(Q)$ is everywhere 0

$$
H=H_{\text {free }}=\frac{P^{2}}{2 \hbar}
$$

Now, by density, we can initially work by only considering values of time $t$ such that $2 \pi t$ is rational. We can then use the Baker-Campbell-Hausdorff formula to compute the following conjugations from the definitions

$$
K^{t} \mathbf{U} K^{-t}=\epsilon^{-\pi t} \mathbf{U V}^{-2 \pi t} \text { and } K^{t} \mathbf{V} K^{-t}=\mathbf{V}
$$

We then define our $\left(\mathbf{U}, \mathbf{V}^{2 \pi t}, K^{t}\right)$-algebra as an algebra generated by these operators and their inverses such that $\mathbf{U}$ and $\mathbf{V}^{2 \pi t}$ satisfy the Weyl commutation relations and $K^{t}$ satsifies these conjugation relations above. Indeed, an operator $K^{t}$ satisfying the conjugation relations will be unique up to multiplication by a normalizing constant over the every finite-dimensional $\left(\mathbf{U}, \mathbf{V}^{2 \pi t}\right)$-module. Note that any $\left(\mathbf{U}, \mathbf{V}^{2 \pi t}\right)$ algebra can be extended to such an algebra by simply adjoining the $K^{t}$ operator with the conjugation relations.

Once again, we note that adjoining the time-propagator to create our $\left(\mathbf{U}, \mathbf{V}^{2 \pi t}, K^{t}\right)$ system will uniquely determine the system up to isomorphism over $\mathcal{C}$, and the system will be a Zariski structure. With all of this setup, we are almost ready to perform our calculations, but we will make some final observations and assumptions. First, we can assume by our structural approximation theorem of quantum tori by tori at roots of unity that $\hbar$ can be treated approximately as a rational number $\frac{M}{N}$ where $\operatorname{gcd}(M, N)=1$ and $N$ divides every feasible integer - where feasible is taken informally to mean that $N$ is some integer with which computations can be performed (maybe 
a number like $\operatorname{lcm}\left(2,3,4, \ldots, 2^{128}-1\right)$ for example). We will also assume that $2 \pi t$ is specifically chosen where its numerator is one of these feasible integers. Furthermore, in mathematical applications, numbers with associated dimensions (meters, seconds, Newtons, etc.). Furthermore, physical units can always be refined by some absorption of constants. In particular, we can always adjust the units of our rational approximation of the reduced Planck constant $\hbar$ so that the constant is replaced, up to change of units, by the quantity $\frac{1}{N}$ for any $N \in \mathbb{Z}^{+}$by absorbing a factor of $\hbar N$ into the units of the reduced Planck constant.

To start the computation, let us look at some of the properties of the $\left(\mathbf{U}, \mathbf{V}^{2 \pi t}, K^{t}\right)$ system we have constucted. Let $\left\{\mathbf{u}(u, v): u \in \mathbb{C}^{*}\right.$ and $\left.v \in R\right\}$ be the system Ueigenvectors, where $R$ is the range of the choice function used in the construction of the $\left(\mathbf{U}, \mathbf{V}^{2 \pi t}\right)$-system. So

$$
\mathbf{U}: \mathbf{u}(u, v) \mapsto u \cdot \mathbf{u}(u, v) \quad \text { and } \quad \mathbf{V}^{2 \pi t}: \mathbf{u}(u, v) \mapsto v^{2 \pi t} \cdot \mathbf{u}(u, v)
$$

Note that the dimension of the modules are $\frac{N}{2 \pi t}$ where this number is an integer by our assumptions about how we are representing the reduced Planck constant. In situations where it is convenient without obscuring the computation, we will abbreviate

$$
M=\frac{N}{2 \pi t}
$$

Let us now denote the following

$$
\mathbf{s}(u, v)=K^{t} \mathbf{u}(u, v) \quad \text { and } \quad S_{t}=\epsilon^{-\pi t} \mathbf{U V}^{-2 \pi t}=K^{t} \mathbf{U} K^{-t}
$$

Therefore we can see, from this conjugation, that for each $u \in \mathbb{C}^{*}, v \in R$, and $k \in \mathbb{Z}$ 


$$
S_{t} \mathbf{s}(u, v)=u \mathbf{s}(u, v) \quad \text { and } \quad \mathbf{V}^{2 \pi k t} \mathbf{s}(u, v)=v^{2 \pi k t} \mathbf{s}\left(\epsilon^{-2 \pi k t} u, v\right)
$$

We can then compute the inner product using the formulas to get

$$
\left(\mathbf{s}(u, v) \mid \mathbf{u}\left(u \epsilon^{2 \pi k t}, v\right)\right)=c \epsilon^{\pi t k^{2}} v^{-2 \pi k t}
$$

where $c$ is a function of $(u, v)$ defined by

$$
c=c\left(u, v^{2 \pi t}\right)=\epsilon^{-\pi t}(\mathbf{s}(u, v) \mid \mathbf{u}(u, v))
$$

It can be show, however, that $c$ does not depend on $(u, v)$ - hence the choice of notation. $c$ only depends on the choice of $\left(\mathbf{U}, \mathbf{V}^{2 \pi t}, K^{t}\right)$.

Next, let us assume that for the moduli of $u$ and $v$ that $|u|=|v|=1$, and that also $\mathbf{U}$ and $\mathbf{V}^{2 \pi t}$ are unitary operators on the inner product space. Then we see there is some $c_{0} \in \mathbb{C}$ with $\left|c_{0}\right|=1$ such that

$$
c=c_{0} \sqrt{\frac{2 \pi t}{N}}
$$

which allows us to express each $\mathbf{s}(u, v)$ in terms of the basis of $\mathbf{u}\left(u \epsilon^{2 \pi k t}, v\right)$ using the inner products in (6.18) and our found value for $c_{0}$

$$
\mathbf{s}(u, v)=c_{0} \sqrt{\frac{N}{2 \pi t}} \sum_{k=0}^{M-1}\left(\epsilon^{\pi t k^{2}} v^{-2 \pi k t} \mathbf{u}\left(u \epsilon^{2 \pi k t}, v\right)\right)
$$

Let us now find a way to solve for $c_{0}$. We can do this by noting that for any $P$-eigenvector $|p\rangle$ with eigenvalue $p$, then $|p\rangle$ is also an eigenvector of 


$$
\exp \left(-i t \frac{P^{2}}{2 \hbar}\right):|p\rangle \mapsto \exp \left(-i t \frac{p^{2}}{2 \hbar}\right)|p\rangle
$$

This means that since $e^{-i t h p^{2}}=\epsilon^{-t p^{2}}$ by (6.13), we note that

$$
K^{t}: \mathbf{v}\left(\epsilon^{2 \pi p t}, 1\right) \mapsto \epsilon^{-\pi t p^{2}} \mathbf{v}\left(\epsilon^{2 \pi p t}, 1\right)
$$

and from this we can recall that in an irreducible $\left(\mathbf{U}, \mathbf{V}^{2 \pi t}\right)$-module, we have the relation

$$
\mathbf{u}(u, 1)=\sum_{p=0}^{\frac{N}{2 \pi t}-1} \mathbf{v}\left(\epsilon^{2 \pi p t}, u\right)
$$

And therefore we have the mapping

$$
K^{t}: \mathbf{u}(1,1) \mapsto \sqrt{\frac{2 \pi t}{N}} \sum_{p=0}^{M-1} \epsilon^{-\pi t p^{2}} \mathbf{v}\left(\epsilon^{2 \pi p t}, 1\right)
$$

The output of this operation, using formula (6.20) with $u=v=1$, is thus

$$
\mathbf{s}(1,1)=K^{t} \mathbf{u}(1,1)=\sqrt{\frac{2 \pi t}{N}} \sum_{p=0}^{M-1} \epsilon^{-\pi t p^{2}} \mathbf{v}\left(\epsilon^{2 \pi p t}, 1\right)
$$

which, upon change of basis of each $\mathbf{v}\left(\epsilon^{2 \pi p t}, 1\right)$ vector using formula $(6.11)$, gives

$$
\begin{aligned}
\mathbf{s}(1,1) & =\sqrt{\frac{2 \pi t}{N}} \sum_{p=0}^{M-1} \epsilon^{-\pi t p^{2}}\left(\sqrt{\frac{2 \pi t}{N}} \sum_{k=0}^{M-1} \epsilon^{2 \pi p k t} \mathbf{u}\left(\epsilon^{2 \pi k t}, 1\right)\right) \\
& =\frac{2 \pi t}{N} \sum_{k=0}^{M-1} \sum_{p=0}^{M-1}\left(\epsilon^{-\pi t\left(p^{2}-2 p k\right)} \mathbf{u}\left(\epsilon^{2 \pi k t}, 1\right)\right)
\end{aligned}
$$

which now, once we equate with our previous general formula for $\mathbf{s}(u, v)$ in terms of the $\mathbf{u}\left(\epsilon^{2 \pi k t} u, v\right)$ basis vectors while setting $u=v=1$, we can equate the coefficients between the two linear combinations and get 


$$
\frac{2 \pi t}{N} \sum_{p=0}^{M-1} \epsilon^{-\pi t\left(p^{2}-2 p k\right)}=c_{0} \sqrt{\frac{2 \pi t}{N}} \epsilon^{\pi t k^{2}}
$$

which for $k=0$ will give us

$$
\sum_{p=0}^{M-1} e^{-i \frac{\pi p^{2}}{M}}=c_{0} \sqrt{M}
$$

where $\epsilon^{-\pi t p^{2}}=e^{-2\left(\pi t p^{2}\right) \pi \hbar}=e^{-i \frac{\pi p^{2}}{M}}$ by (6.13). We note that the left-hand side of this equation is a Gauss sum from analytic number theory if $M$ is an even integer, which we will go ahead and assume, to obtain

$$
\sqrt{M} c_{0}=\sum_{p=0}^{M-1} e^{-i \frac{\pi p^{2}}{M}}=\frac{1-i}{\sqrt{2}} \sqrt{M}
$$

which finally gives us that

$$
c_{0}=\frac{1-i}{\sqrt{2}}
$$

Finally, we will compute the kernel of the Feynman propagator of our free particle system. This will be defined as

$$
\left\langle x_{1}\left|K^{t}\right| x_{0}\right\rangle
$$

where each $\left|x_{i}\right\rangle$ represents a position eigenvector, and the formula should be read to mean the inner product of $K^{t}\left|x_{0}\right\rangle$ and $\left|x_{1}\right\rangle$.

We note that $e^{2 \pi i x_{i}}=\epsilon^{\frac{x_{i}}{\hbar}}$. Let us assume each $\frac{x_{i}}{\hbar}$ as a rational number and rewrite our Feynman propagator as

$$
\frac{N}{2 \pi t} \cdot\left(\mathbf{s}\left(\epsilon^{\frac{x_{0}}{\hbar}}, 1\right) \mid \mathbf{u}\left(\epsilon^{\frac{x_{1}}{\hbar}}, 1\right)\right)
$$

We now use our inner product formula with $x_{0}=2 \pi t m \hbar, u=\epsilon^{2 \pi t m}, x_{1}=2 \pi(m+k) t \hbar$, and $u \epsilon^{2 \pi k t}=\epsilon^{2 \pi(m+k) t}$ to get 


$$
\left(\mathbf{s}\left(\epsilon^{\frac{x_{0}}{\hbar}}, 1\right) \mid \mathbf{u}\left(\epsilon^{\frac{x_{1}}{\hbar}}, 1\right)\right)=c_{0} \sqrt{\frac{2 \pi t}{N}} e^{i \frac{\left(x_{1}-x_{0}\right)^{2}}{2 t \hbar}}
$$

but we have already demonstrated that $c_{0}=\frac{1-i}{\sqrt{2}}=\sqrt{-i}=\sqrt{\frac{1}{i}}$, and therefore

$$
\left\langle x_{1}\left|K^{t}\right| x_{0}\right\rangle=\sqrt{\frac{1}{2 \pi i \hbar t}} e^{i \frac{\left(x_{1}-x_{0}\right)^{2}}{2 t \hbar}}
$$

is the kernel of the Feynman propagator for a free particle. This coincides with the formulas obtained from the usual heuristics obtained in physics, as can be seen in [10] as formula (7.76). The usual method to obtain the result would involve considering the Gaussian integral

$$
I(a)=\int_{\mathbb{R}} e^{-a \frac{x^{2}}{2}} e^{-i p x} d x
$$

and assign some meaning to the integral for $a=\frac{i}{t h}$. The integral is divergent for imaginary values of $a$, but it converges for $a$ in the right half-plane of $\mathbb{C}$. This computation is performed for $a$ by taking the analytic continuation of $I(a)$ from the right half-plane to its boundaries.

Along with this result, Zilber shows that much of the same methodology can be applied to also compute the kernel of the Feynman propagator for the case where the Hamiltonian operator that of the simple Harmonic oscillator

$$
H_{o s c}=\frac{P^{2}+\omega^{2} Q^{2}}{2 \hbar}
$$

This result can be found in [13].

To summarize the process taken again, the tools we have developed for studying nonclassical Zariski structures were able to be adapted to the setting of quantum mechanics by finding some Zariski structures which could help us model our quantum 
state spaces, and then isolating a family of nice Zariski structures which essentially had a family of self-dual modules as its fibers at each point over their classical geometrical spaces (particularly, the quantum tori at roots of unity). We then developed a notion of structural approximation from the notion of ultraproducts from model theory, which gave us a result which said our setting of quantum mechanics could be obtained by deforming a family of quantum tori to represent the space, thus giving us the ability to approximately represent our quantum mechanical model in terms of quantum tori. Then finally, after some grinding through inner product computations with a newly-adjoined time-propagator operator, we developed a technique which has allowed us to obtain the same formulas that heuristic physics methods obtain, but in a fairly mathematically rigorous fashion. 


\section{CHAPTER 7}

\section{CONCLUDING REMARKS}

After all this said about Zariski geometries and their applications to quantum mechanics, the work presented here only scratches the surface of the theory of Zariski structures and Zilber's trichotomy principle. Not only have Zariski geometries been applied by Hrushovski to prove the case of the Mordell-Lang conjecture for function fields [3], but there has also been work to apply the theory of Zariski structures to differential closed fields, such as Pillay's theorem regarding finite Morley rank subsets of differentially closed fields [7]. Zilber's trichotomy principle has also seemingly inspired works in the classification of other kinds of minimal structures, such as a form of trichotomy theorem proven by Peterzil and Starchenko about o-minimal structures - lineared ordered structures whose definable sets are reducible to Boolean combinations of points and intervals [6].

The applications of Zariski structures to quantum mechanics is also still a work in progress by Zilber, and some of his most recent work on the matter can be found in [16], where the technique of structural approximation is developed in more robust ways, and with an open avenue of research being to find ways to adapt these techniques to computing propagators for higher-order polynomials expressing the potential of a particle. 


\section{REFERENCES}

[1] Wilfrid Hodges. A shorter model theory. Cambridge University Press, Cambridge, 1997.

[2] Ehud Hrushovski. A new strongly minimal set. volume 62, pages 147-166. 1993. Stability in model theory, III (Trento, 1991).

[3] Ehud Hrushovski. The Mordell-Lang conjecture for function fields. J. Amer. Math. Soc., 9(3):667-690, 1996.

[4] Ehud Hrushovski and Boris Zilber. Zariski geometries. J. Amer. Math. Soc., $9(1): 1-56,1996$.

[5] David Marker. Model theory, volume 217 of Graduate Texts in Mathematics. Springer-Verlag, New York, 2002. An introduction.

[6] Ya'acov Peterzil and Sergei Starchenko. A trichotomy theorem for o-minimal structures. Proc. London Math. Soc. (3), 77(3):481-523, 1998.

[7] Anand Pillay. Two remarks on differential fields. In Model theory and applications, volume 11 of Quad. Mat., pages 325-347. Aracne, Rome, 2002.

[8] Jeffrey M. Rabin. Introduction to quantum field theory for mathematicians. In Geometry and quantum field theory (Park City, UT, 1991), volume 1 of IAS/Park City Math. Ser., pages 183-269. Amer. Math. Soc., Providence, RI, 1995. 
[9] Igor R. Shafarevich. Basic algebraic geometry. 1. Springer, Heidelberg, third edition, 2013. Varieties in projective space.

[10] Eberhard Zeidler. Quantum field theory. II. Quantum electrodynamics. SpringerVerlag, Berlin, 2009. A bridge between mathematicians and physicists.

[11] Boris Zilber. The structure of models of uncountably categorical theories. In Proceedings of the International Congress of Mathematicians, Vol. 1, 2 (Warsaw, 1983), pages 359-368. PWN, Warsaw, 1984.

[12] Boris Zilber. A class of quantum Zariski geometries. In Model theory with applications to algebra and analysis. Vol. 1, volume 349 of London Math. Soc. Lecture Note Ser., pages 293-326. Cambridge Univ. Press, Cambridge, 2008.

[13] Boris Zilber. On model theory, non-commutative geometry and physics. 2010.

[14] Boris Zilber. Zariski geometries, volume 360 of London Mathematical Society Lecture Note Series. Cambridge University Press, Cambridge, 2010. Geometry from the logician's point of view.

[15] Boris Zilber. Perfect infinities and finite approximation. In Infinity and truth, volume 25 of Lect. Notes Ser. Inst. Math. Sci. Natl. Univ. Singap., pages 199-223. World Sci. Publ., Hackensack, NJ, 2014.

[16] Boris Zilber. Structural approximation and quantum mechanics (draft). 2019. 